\title{
Unconditional uniqueness for the energy-critical nonlinear Schrödinger equation on $\mathbb{T}^{4}$
}

\author{
Xuwen Chen ${ }^{1}$ and Justin Holmer ${ }^{(\mathbb{D})}$ \\ ${ }^{1}$ Department of Mathematics, University of Rochester, Hylan Building, Rochester, NY 14627, USA; \\ E-mail: chenxuwen@math.umd.edu. \\ ${ }^{2}$ Department of Mathematics, Brown University, 151 Thayer Street, Providence, RI 02912, USA; \\ E-mail: justin_holmer@brown.edu.
}

Received: 2 April 2021; Accepted: 26 October 2021

2020 Mathematics Subject Classification: Primary - 35Q55, 35A02, 81V70; Secondary - 35A23, 35B45, 81Q05

\begin{abstract}
We consider the $\mathbb{T}^{4}$ cubic nonlinear Schrödinger equation (NLS), which is energy-critical. We study the unconditional uniqueness of solutions to the NLS via the cubic Gross-Pitaevskii hierarchy, an uncommon method for NLS analysis which is being explored $[24,35]$ and does not require the existence of a solution in Strichartz-type spaces. We prove $U-V$ multilinear estimates to replace the previously used Sobolev multilinear estimates. To incorporate the weaker estimates, we work out new combinatorics from scratch and compute, for the first time, the time integration limits, in the recombined Duhamel-Born expansion. The new combinatorics and the $U-V$ estimates then seamlessly conclude the $H^{1}$ unconditional uniqueness for the NLS under the infinite-hierarchy framework. This work establishes a unified scheme to prove $H^{1}$ uniqueness for the $\mathbb{R}^{3} / \mathbb{R}^{4} / \mathbb{T}^{3} / \mathbb{T}^{4}$ energy-critical Gross-Pitaevskii hierarchies and thus the corresponding NLS.
\end{abstract}

\section{Contents}

1 Introduction $\quad 2$

1.1 Outline of the proof of Theorem $1.1 \ldots \ldots \ldots \ldots$

2 Trilinear estimates in the $U-V$ spaces $\quad 6$

2.1 Proof of Lemma $2.1 \ldots \ldots \ldots \ldots \ldots$

3 Uniqueness for GP hierarchy (1.2) and the proof of Theorem 1.1 - Setup 10

3.1 Proof of Lemma 3.3 and uniform-in-time frequency localisation for the NLS . . . . 15

4 An extended KM board game $\quad 16$

4.1 A more elaborated proof of Lemma $3.6 \ldots \ldots \ldots$. . . . . . . . . . . . . . . . . . . . . . .

4.2 Signed KM acceptable moves . . . . . . . . . . . . . . . . . . . . 22

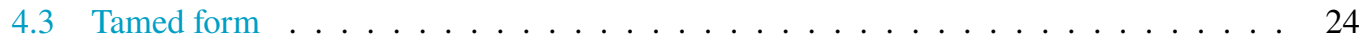

4.4 Wild moves . . . . . . . . . . . . . . . . . . . . . 31

4.5 Reference forms and tamed integration domains . . . . . . . . . . . . 35

$5 \quad$ Uniqueness for the GP hierarchy (1.2) - Actual estimates $\quad 37$

5.1 An example of how to estimate . . . . . . . . . . . . . . . 38

5.2 The extended KM board game is compatible . . . . . . . . . . . . . . 40

5.3 Estimates for general $k \ldots \ldots \ldots \ldots \ldots$

$\begin{array}{lll}\text { Appendix A Some further remarks } & 45\end{array}$

(c) The Author(s), 2022. Published by Cambridge University Press. This is an Open Access article, distributed under the terms of the Creative Commons Attribution licence (https://creativecommons.org/licenses/by/4.0/), which permits unrestricted re-use, distribution, and reproduction in any medium, provided the original work is properly cited. 


\section{Introduction}

The cubic nonlinear Schrödinger equation (NLS) in four dimensions

$$
\begin{aligned}
i \partial_{t} u & =-\Delta u \pm|u|^{2} u \text { in } \mathbb{R} \times \Lambda, \\
u(0, x) & =u_{0},
\end{aligned}
$$

where $\Lambda=\mathbb{R}^{4}$ or $\mathbb{T}^{4}$, is called energy-critical, because it is invariant under the $\dot{H}^{1}$ scaling

$$
u(t, x) \mapsto u_{\lambda}(t, x)=\frac{1}{\lambda} u\left(\frac{t}{\lambda^{2}}, \frac{x}{\lambda}\right)
$$

if $\Lambda=\mathbb{R}^{4}$. The large-datum global well-posedness of the defocusing case of equation (1.1) was first proved for $\Lambda=\mathbb{R}^{4}$ in [56], after the breakthrough on the defocusing $\mathbb{R}^{3}$ quintic problem [3, 28, 33]. The argument was standardised in [42], in which the radial focusing $\mathbb{R}^{3}$ quintic problem was addressed. After that, the global well-posedness of the energy-critical defocusing $\mathbb{T}^{3}$ quintic problem was creatively settled in $[36,40]$, by partially invoking the $\mathbb{R}^{3}$ result $[28,42]$. Such a problem for equation (1.1) when $\Lambda=\mathbb{T}^{4}$ was then subsequently proved in [37, 43, 61]. The goal of this paper is to establish $H^{1}$ unconditional uniqueness for equation (1.1) on $\mathbb{T}^{4}$.

Theorem 1.1. There is at most one $C_{[0, T]}^{0} H_{x}^{1} \cap \dot{C}_{[0, T]}^{1} H_{x}^{-1}$ solution ${ }^{1}$ to equation (1.1) on $\mathbb{T}^{4} .^{2}$

Unconditional uniqueness is a fundamental concept raised by Kato [41]. ${ }^{3}$ These problems, even in the $H^{1}$-critical setting, are often overlooked, as solving them in $\mathbb{R}^{n}$ after proving the well-posedness is relatively simple. ${ }^{4}$ For the NLS on $\mathbb{T}^{n}$, such problems are delicate, as estimates on $\mathbb{T}^{n}$, especially the $\mathbb{T}^{n}$ Strichartz estimates, are weaker than their $\mathbb{R}^{n}$ counterparts. For example, for the $\mathbb{R}^{n}$ case, one can easily use the existence of a better solution in Strichartz spaces to yield unconditional uniqueness. But such a technique does not work well in the $\mathbb{T}^{n}$ case. In fact, Theorem 1.1 for the $\mathbb{T}^{3}$ quintic case at $H^{1}$ regularity was not known until recently [24].

To prove Theorem 1.1, we will use the cubic Gross-Pitaevskii (GP) hierarchy on $\mathbb{T}^{4}$, which is uncommon in the analysis of the NLS and is being explored [24, 35]. Let $\mathcal{L}_{k}^{1}$ denote the space of trace class operators on $L^{2}\left(\mathbb{T}^{4 k}\right)$. The cubic GP hierarchy on $\mathbb{T}^{4}$ is a sequence $\left\{\gamma^{(k)}(t)\right\} \in \oplus_{k \geq 1} C\left([0, T], \mathcal{L}_{k}^{1}\right)$ which satisfies the infinitely coupled hierarchy of equations

$$
i \partial_{t} \gamma^{(k)}=\sum_{j=1}^{k}\left[-\Delta_{x_{j}}, \gamma^{(k)}\right] \pm b_{0} \sum_{j=1}^{k} \operatorname{Tr}_{k+1}\left[\delta\left(x_{j}-x_{k+1}\right), \gamma^{(k+1)}\right]
$$

where $b_{0}>0$ is some coupling constant and \pm denotes defocusing/focusing. Given any solution $u$ of equation (1.1), we generate a solution to equation (1.2) by letting

$$
\gamma^{(k)}=|u\rangle\left\langle\left. u\right|^{\otimes k},\right.
$$

in operator form, or

$$
\gamma^{(k)}\left(t, \mathbf{x}_{k}, \mathbf{x}_{k}^{\prime}\right)=\prod_{j=1}^{k} u\left(t, x_{j}\right) \bar{u}\left(t, x_{j}^{\prime}\right)
$$

in kernel form, if we write $\mathbf{x}_{k}=\left(x_{1}, \ldots, x_{k}\right) \in \mathbb{T}^{4 k}$.

\footnotetext{
${ }^{1} \mathrm{~A} C_{[0, T]}^{0} H_{x}^{1}$ distributional solution is automatically a $C_{[0, T]}^{0} H_{x}^{1} \cap \dot{C}_{[0, T]}^{1} H_{x}^{-1}$ solution. We wrote the latter here because it is a more direct space for equation (1.1).

${ }^{2}$ See also Theorem A.1 for another format, closer to well-posedness theory, of Theorem 1.1; its proof shows that every $C_{[0, T]}^{0} H_{x}^{1}$ solution is also in $X^{1}$ without using any previous well-posedness results.

${ }^{3}$ According to C. Miao, Kato wrote a letter to him, accompanying a book, mentioning these unconditional-uniqueness problems at critical settings one month before passing away.

${ }^{4}$ See, for example, [28, Section 16].
} 
The hierarchy (1.2) arises in the derivation of the NLS as an $N \rightarrow \infty$ limit of quantum $N$-body dynamics. It was first derived in the work of Erdös, Schlein and Yau [29, 30, 31] for the $\mathbb{R}^{3}$ defocusing cubic case around $2005 .{ }^{5}$ They proved delicately that there is a unique solution to the $\mathbb{R}^{3}$ cubic GP hierarchy in an $H^{1}$-type space (unconditional uniqueness) in [29], with a sophisticated Feynman-graph analysis. This first series of groundbreaking papers motivated a large amount of work.

In 2007, Klainerman and Machedon [47], inspired by [29, 46], proved the uniqueness of solutions regarding the $\mathbb{R}^{3}$ cubic GP hierarchy in a Strichartz-type space (conditional uniqueness). They proved a collapsing-type estimate, which implies a multilinear estimate when applied to factorised solutions like equation (1.3), to estimate the inhomogeneous term and provided a different combinatorial argument, now called the Klainerman-Machedon (KM) board game, to combine the inhomogeneous terms, effectively reducing their numbers. At that time, it was unknown how to prove that the limits coming from the $N$-body dynamics are in Strichartz-type spaces, even though the solutions to equation (1.2) generated by the $\mathbb{R}^{3}$ cubic NLS naturally lie in both the $H^{1}$-type space and the Strichartz-type space. Nonetheless, [47] has made the analysis of equation (1.2) approachable to partial-differential-equation analysts, and the KM board game has been used in every work involving the hierarchy (1.2). ${ }^{6}$ After Kirkpatrick, Schlein and Staffilani [44] derived equation (1.2) in 2008 and found that the Klainerman-Machedon Strichartz-type bound can be obtained via a simple trace theorem for the defocusing case in $\mathbb{R}^{2}$ and $\mathbb{T}^{2}$, many works $[8,14,16,18,20,22,23,60,58]$ then followed such a scheme for the uniqueness of GP hierarchies. However, the question of how to check the Klainerman-Machedon Strichartz-type bound in the $3 \mathrm{D}$ cubic case remained fully open at that time.

T. Chen and Pavlović laid the foundation for the 3D quintic defocusing energy-critical case by studying the $1 \mathrm{D}$ and 2D defocusing quintic cases in [8], in which they proved that the 2D quintic case, a case usually considered equivalent to the 3D cubic case, does satisfy the Klainerman-Machedon Strichartz-type bound - although proving it for the 3D cubic case was still open.

T. Chen and Pavlović also initiated the study of the well-posedness theory of equation (1.2) with general initial datum as an independent subject away from the quantum $N$-body dynamics in [7, 9, 10] (see also [12, 13, 55, 53, 54, 58, 59]). On the one hand, generalising the problem could help to attack the Klainerman-Machedon Strichartz-type bound problem. On the other hand, it leads one to consider whether the hierarchy (1.2), the general equation, could hold more in store than its special solution, the NLS (equation (1.1)). ${ }^{7}$ Then in 2011, T. Chen and Pavlović proved that the 3D cubic KlainermanMachedon Strichartz-type bound does hold for the defocusing $\beta<1 / 4$ case [11]. The result was quickly improved to $\beta \leq 2 / 7$ by X. Chen [17] and to the almost-optimal case, $\beta<1$, by X. Chen and Holmer $[19,21]$, by lifting the $X_{1, b}$ space techniques from NLS theory into the field.

Around the same time, Gressman, Sohinger and Staffilani [32] studied the uniqueness of equation (1.2) in the $\mathbb{T}^{3}$ setting and found that the sharp collapsing estimate on $\mathbb{T}^{3}$ needs $\varepsilon$ more derivatives than the $\mathbb{R}^{3}$ case, in which one derivative is needed. Herr and Sohinger later generalised this fact to all dimensions [34] - that is, collapsing estimates on $\mathbb{T}^{n}$ always need $\varepsilon$ more derivatives than the $\mathbb{R}^{n}$ case proved in [16]. ${ }^{8}$

In 2013, T. Chen, Hainzl, Pavlović and Seiringer introduced the quantum de Finetti theorem, from [51], to the derivation of the time-dependent power-type NLS and provided, in [6], a simplified proof of the $\mathbb{R}^{3}$ unconditional uniqueness theorem regarding equation (1.2) from [29]. The application of the quantum de Finetti theorem allows one to replace the collapsing estimates by the multilinear estimates. The scheme in [6], which consists of the KM board game, the quantum de Finetti theorem and the multilinear estimates, is robust. Sohinger used this scheme in [57] to address the aforementioned $\varepsilon$-loss problem for the defocusing $\mathbb{T}^{3}$ cubic case. Hong, Taliaferro and Xie used this scheme in [38] to obtain unconditional uniqueness theorems for equation (1.2) in $\mathbb{R}^{n}, n=1,2,3$, with regularities matching the NLS analysis, and in [39] for $H^{1}$ small-solution uniqueness in the $\mathbb{R}^{3}$ quintic case (see also [22, 27]).

\footnotetext{
${ }^{5}$ See also [1] for the 1D defocusing cubic case around the same time.

${ }^{6}$ Analysis of the Boltzmann hierarchy can also use the KM board game; see, for example, [5].

${ }^{7}$ Private communication with T. Chen and Pavlović.

${ }^{8}$ Except in the $1 \mathrm{D}$ case, as shown in [20], this $\varepsilon$ loss also happens in $\mathbb{R}^{1}$.
} 
Analysis of GP hierarchy did not yield new NLS results with regularity lower than that of NLS analysis until [35, 24]. ${ }^{9,10}$ In [35], using the scheme in [6], Herr and Sohinger generalised the usual Sobolev multilinear estimates to Besov spaces and obtained new unconditional-uniqueness results regarding equation (1.2) and hence the NLS (equation (1.1)) on $\mathbb{T}^{n}$. The result has pushed the regularity requirement for the uniqueness of equation (1.1) lower than the number coming from NLS analysis. Moreover, it covers the whole subcritical region for $n \geq 4$, which includes Theorem 1.1 with $H^{1+\varepsilon}$ regularity.

In [24], by discovering the new hierarchical uniform frequency localisation (HUFL) property for the GP hierarchy - which reduces to a new statement even for the NLS - X.C. and Holmer established a new $H^{1}$-type uniqueness theorem for the $\mathbb{T}^{3}$ quintic energy-critical GP hierarchy. ${ }^{11}$ The new uniqueness theorem, though neither conditional nor unconditional for the GP hierarchy, implies the $H^{1}$ unconditional-uniqueness result for the $\mathbb{T}^{3}$ quintic energy-critical NLS. It is then natural to consider the $\mathbb{T}^{4}$ cubic energy-critical case in this paper. However, the key Sobolev multilinear estimates in [24] are very difficult to prove, or may not be true, for the $\mathbb{T}^{4}$ cubic case here, and it turns out, surprisingly, that $\mathbb{T}^{4}$ is unique or special compared to $\mathbb{R}^{3} / \mathbb{R}^{4} / \mathbb{T}^{3}$.

\subsection{Outline of the proof of Theorem 1.1}

We will prove Theorem 1.1 as a corollary of Theorem 3.1, a GP-hierarchy uniqueness theorem stated in Section 3. As Theorem 3.1 requires the HUFL condition, we prove that any $C_{[0, T]}^{0} H_{x}^{1} \cap \dot{C}_{[0, T]}^{1} H_{x}^{-1}$ solution to equation (1.1) on $\mathbb{T}^{4}$ satisfies uniform-in-time frequency localisation with Lemma 3.3 that is, solutions to equation (1.2) generated from equation (1.1) via equation (1.3) satisfy the HUFL condition. Thus we will have established Theorem 1.1 once we have proved Theorem 3.1.

As Theorem 3.1 is an energy-critical case, due to the known similarities between the $\mathbb{R}^{3}$ quintic and $\mathbb{R}^{4}$ cubic cases, one would guess that the proof of the $\mathbb{T}^{3}$ quintic case goes through for the $\mathbb{T}^{4}$ cubic case as well. It does not. As mentioned before, the key Sobolev multilinear estimates in [24] are very difficult to prove, or may not be true here (interested readers can see Appendix A for a discussion). In this $H^{1}$-critical setting, the next replacement in line would be the weaker $U-V$ multilinear estimates. The $U-V$ trilinear estimates do hold on $\mathbb{T}^{4}$. This is where we start.

In Section 2, we first give a short introduction to the $U-V$ space, referring the standard literature $[36,40,43,50]$, then prove the $U-V$ version of the $\mathbb{T}^{4}$ trilinear estimates (Lemmas 2.1 and 2.2). The proof of the $U-V$ trilinear estimates is less technical and simpler than the proof of the Sobolev multilinear estimates in [24], as they are indeed weaker. ${ }^{12}$ (As we will conclude the same unconditonal uniquness with these much weaker estimates, we can infer that our method here is indeed much stronger now.) But these $U-V$ trilinear estimates still highly rely on the scale-invariant Stichartz estimates and the $l^{2}$-decoupling theorem in $[4,43]$.

Though the $U-V$ trilinear estimates hold in $\mathbb{T}^{4}$, there is no method available to use them to prove uniqueness for GP hierarchies. This is why estimates in the hierarchy framework have always been about $L_{t}^{p} H_{x}^{s}$. Even in $[19,21]$, in which the $X_{s, b}$ techniques were used, they were used only once in the very end of the iteration, instead of every step of the iteration to yield smallness. Conceptually speaking, while it is easy to bound the $L_{t}^{\infty} H_{x}^{s}$ norm by the $U-V$ norms, one has to pay half a derivative in time to come back. On the one hand, we are proving an unconditional-uniqueness theorem; we have to come back to the Sobolev spaces in the end of the proof. On the other hand, we are proving a critical result; we do not have an extra half derivative in time to spare. To fix this problem, we adjust how the multilinear estimates apply to the Duhamel-Born expansion of $\gamma^{(k)}$ after the application of the KM board game, so that the $U-V$ trilinear estimates land only on a 'Duhamel-like' integral.

\footnotetext{
${ }^{9}$ Here we mention [35] before [24], even though [24] was posted on arXiv a month before [35], because we were not aware of the unconditional-uniqueness outcome of [24] until [35].

${ }^{10}$ See also [45] for recent developments using NLS analysis.

${ }^{11}$ See [17] for another type of quintic problems.

${ }^{12}$ The stronger Sobolev multilinear estimates hold, easily, for $\mathbb{R}^{4}$ (see Appendix A).
} 
The main problem now surfaces. The time-integration domain $D_{m}$ of the aforementioned 'Duhamellike' integrals, coming from the KM board game, is a union of a very large number of high-dimensional simplexes under the action of a proper subset of the permutation group $S_{k}$ specific to every integrand. To at least have a chance to use space-time norms like $X_{s, b}$ and $U-V$ - which are very sensitive to the irregularity of the time domain, as they involve taking time derivatives [50, p. 68] - one would have to know what $D_{m}$ is. It turns out that $D_{m}$ coming from the original KM board game is not fully compatible with the $U-V$ trilinear estimates. To this end, we establish an extended KM board game which is compatible in Section 4.

In Section 4.1, as a warm-up, we first develop - via a detailed tree ${ }^{13}$ diagram representation - a more elaborated proof of the original KM board game, which yields, for the first time, an algorithm to directly compute $D_{m}$ and domains like it. Graphically speaking, under our tree representation the original KM board game combines all the trees with the same skeletons into an 'upper-echelon' class which can be represented by an upper-echelon tree. ${ }^{14}$ The time integration domain $D_{m}$ for each upper-echelon class can be directly read off from the upper-echelon tree representing the class.

We then introduce, in Sections 4.2-4.5, the wild moves, which allow us to uncover more integrals in the Duhamel-Born expansion with the same integrands after permutation and combine them into 'reference' classes. Graphically speaking, it allows the combination of trees sharing the same reference enumeration but with different structures. However, the wild moves are not compatible with the upperechelon classes coming from the original KM board game. We have to restart from the very beginning at the level of the $2^{k} k$ ! summands.

Before applying the wild moves, in Sections 4.2 and 4.3 we turn the $2^{k} k$ ! summands in the initial Duhamel-Born expansion into their tamed forms, which would be invariant under the wild moves, via reworked signed Klainerman-Machedon acceptable moves. We then sort the tamed forms into tamed classes via the wild moves in Section 4.4. Finally, in Section 4.5 we use the algorithm developed in Section 4.1 to calculate the time integration domain for each tamed class. In fact, we prove that given a tamed class, there is a reference form representing the tamed class, and the time integration domain for the whole tamed class can be directly read out from the reference form.

Using this extended KM board game coming from scratch, we found that the time integration domain specific for each integrand can always be 'miraculously' written as one single iterated integral in the integration order ready to apply the quantum de Finetti theorem, despite the fact that it was previously thought unrepresentable or even disconnected, and was expanded into $[0, T]^{k}$ in all previous work since there were no other options to use it. Moreover, once these integration limits are put together with the integrand, each distinct tamed class becomes an exact fit to apply the $U-V$ trilinear estimates proved in Section 2. This combinatorial analysis, which is compatible with space-time norms and the method to explicitly compute the time integration domain in the general recombined Duhamel-Born expansion (which includes more than the GP hierarchies), is the main technical achievement of this paper.

With everything ready by the extended KM board game in Section 4, the quantum de Finetti theorem from [6], the $U-V$ space techniques from [50], the trilinear estimates proved using the scale-invariant Stichartz estimates and $l^{2}$-decoupling theorem in [4, 43] and the HUFL properties from [24], all work together seamlessly in Section 5 to establish Theorem 3.1 and provide a unified proof of large-solution uniqueness for the $\mathbb{R}^{3} / \mathbb{T}^{3}$ quintic and the $\mathbb{R}^{4} / \mathbb{T}^{4}$ cubic energy-critical GP hierarchies, and hence the corresponding NLS. The discovery of such an unexpected close and effective collaboration of these previously independent deep theorems is the main novelty of this paper.

We remark that putting together Theorem 3.1 and the compactness and convergence argument of [24] completes a derivation of equation (1.1) from quantum many-body dynamics. We choose not to do so here, as it is not the main point of this paper. We now expect to be able to bring the full strength of

\footnotetext{
${ }^{13}$ This is the third type of tree used in the analysis of GP hierarchies. The first two are the Feymann graphs in [29] and the binary trees in [6]. They are coded differently and serve different purposes.

${ }^{14}$ It is possible to write Section 4 without trees (or matrices), but we would lose this graphical explanation. Due to the coupling, recursive and iterative features of the hierarchies, algorithm terminologies happen to be helpful.
} 
dispersive estimate technology to bear on various type of hierarchies of equations and related problems, and this is our first example of it. (An immediate next step has been taken [26].)

\section{Trilinear estimates in the $U-V$ spaces}

As mentioned in the introduction, our proof of Theorem 1.1 requires the $U-V$ space, whereas the $\mathbb{R}^{3} / \mathbb{R}^{4} / \mathbb{T}^{3}$ cases do not. Here $V^{p}$ is the space of functions of bounded $p$-variation of Wiener, and the atomic $U^{p}$ space, introduced by Koch and Tataru [48, 49], is a close relative of $V^{p}$. Referring to the now-standard text [50] for the definition of $U_{t}^{p}$ and $V_{t}^{p}$, we define

$$
\left.\|u\|_{X^{s}([0, T))}=\left(\sum_{\xi \in \mathbb{Z}^{4}}\langle\xi\rangle^{2 s} \| e^{-\overline{i t \Delta} u(t,}\right)(\xi) \|_{U_{t}^{2}}^{2}\right)^{\frac{1}{2}}
$$

and

$$
\left.\|u\|_{Y^{s}([0, T))}=\left(\sum_{\xi \in \mathbb{Z}^{4}}\langle\xi\rangle^{2 s} \| e^{-\overline{i t \Delta} u(t,} \cdot\right)(\xi) \|_{V_{t}^{2}}^{2}\right)^{\frac{1}{2}}
$$

as in $[36,37,40,43] . X^{s}$ and $Y^{s}$ are endpoint replacements for the Fourier restriction spaces $X^{s, b}$ when $b=\frac{1}{2}$ and $-\frac{1}{2}$. In particular, we have the usual properties

$$
\begin{gathered}
\|u\|_{L_{t}^{\infty} H_{x}^{s} \lesssim\|u\|_{X^{s}}}\left\|e^{i t \Delta} f\right\|_{Y^{s}} \lesssim\left\|e^{i t \Delta} f\right\|_{X^{s}} \lesssim\|f\|_{H^{s}} \\
\left\|\int_{a}^{t} e^{i(t-s) \Delta} f(s, \cdot) d s\right\|_{X^{s}([0, T))} \leq \sup _{v \in Y^{-s}([0, T)):\|v\|_{Y^{-s}=1}} \int_{0}^{T} \int_{\mathbb{T}^{4}} f(t, x) \overline{v(t, x)} d t d x \quad \forall a \in[0, T),
\end{gathered}
$$

which were proved in [50, p. 46] and in [36, Propositions 2.8-2.11]. With these definitions of $X^{s}$ and $Y^{s}$, we have the following trilinear estimates:

Lemma 2.1. On $\mathbb{T}^{4}$, we have the high frequency estimate

$$
\iint_{x, t} u_{1}(t, x) u_{2}(t, x) u_{3}(t, x) g(t, x) d x d t \lesssim\left\|u_{1}\right\|_{Y^{-1}}\left\|u_{2}\right\|_{Y^{1}}\left\|u_{3}\right\|_{Y^{1}}\|g\|_{Y^{1}}
$$

and the low frequency estimate

$$
\iint_{x, t} u_{1}(t, x)\left(P_{\leq M_{0}} u_{2}\right)(t, x) u_{3}(t, x) g(t, x) d x d t \lesssim T^{\frac{1}{7}} M_{0}^{\frac{3}{5}}\left\|u_{1}\right\|_{Y^{-1}}\left\|P_{\leq M_{0}} u_{2}\right\|_{Y^{1}}\left\|u_{3}\right\|_{Y^{1}}\|g\|_{Y^{1}}
$$

for all $T \leq 1$ and all frequencies $M_{0} \geq 1$, or

$$
\left\|\int_{a}^{t} e^{i(t-s) \Delta}\left(u_{1} u_{2} u_{3}\right) d s\right\|_{X^{-1}([0, T))} \lesssim\left\|u_{1}\right\|_{Y^{-1}}\left(T^{\frac{1}{7}} M_{0}^{\frac{3}{5}}\left\|P_{\leq M_{0}} u_{2}\right\|_{Y^{1}}+\left\|P_{>M_{0}} u_{2}\right\|_{Y^{1}}\right)\left\|u_{3}\right\|_{Y^{1}}
$$

and

$$
\left\|\int_{a}^{t} e^{i(t-s) \Delta}\left(u_{1} u_{2} u_{3}\right) d s\right\|_{X^{-1}([0, T))} \lesssim\left\|u_{1}\right\|_{Y^{-1}}\left\|u_{2}\right\|_{Y^{1}}\left\|u_{3}\right\|_{Y^{1}} .
$$


Moreover, if $u_{j}=e^{i t \Delta} f_{j}$ for some $j$, then the $Y^{s}$ norm of $u_{j}$ in formula (2.6) or (2.7) can be replaced by the $H^{s}$ norm of $f_{j}$.

Similarly, we have $X^{1}$ estimates:

Lemma 2.2. On $\mathbb{T}^{4}$, we have the high frequency estimate

$$
\iint_{x, t} u_{1}(t, x) u_{2}(t, x) u_{3}(t, x) g(t, x) d x d t \lesssim\left\|u_{1}\right\|_{Y^{1}}\left\|u_{2}\right\|_{Y^{1}}\left\|u_{3}\right\|_{Y^{1}}\|g\|_{Y^{-1}}
$$

and the low frequency estimate

$$
\iint_{x, t} u_{1}(t, x)\left(P_{\leq M_{0}} u_{2}\right)(t, x) u_{3}(t, x) g(t, x) d x d t \lesssim T^{\frac{1}{7}} M_{0}^{\frac{3}{5}}\left\|u_{1}\right\|_{Y^{1}}\left\|P_{\leq M_{0}} u_{2}\right\|_{Y^{1}}\left\|u_{3}\right\|_{Y^{1}}\|g\|_{Y^{-1}}
$$

In other words,

$$
\left\|\int_{a}^{t} e^{i(t-s) \Delta}\left(u_{1} u_{2} u_{3}\right) d s\right\|_{X^{1}([0, T))} \lesssim\left\|u_{1}\right\|_{Y^{1}}\left(T^{\frac{1}{7}} M_{0}^{\frac{3}{5}}\left\|P_{\leq M_{0}} u_{2}\right\|_{Y^{1}}+\left\|P_{>M_{0}} u_{2}\right\|_{Y^{1}}\right)\left\|u_{3}\right\|_{Y^{1}}
$$

and

$$
\left\|\int_{a}^{t} e^{i(t-s) \Delta}\left(u_{1} u_{2} u_{3}\right) d s\right\|_{X^{1}([0, T))} \lesssim\left\|u_{1}\right\|_{Y^{1}}\left\|u_{2}\right\|_{Y^{1}}\left\|u_{3}\right\|_{Y^{1}} .
$$

Moreover, if $u_{j}=e^{i t \Delta} f_{j}$ for some $j$, then the $Y^{s}$ norm of $u_{j}$ in formula (2.10) or (2.11) can be replaced by the $H^{s}$ norm of $f_{j}$.

We prove only Lemma 2.1. On the one hand, Lemma 2.2 follows from the proof of Lemma 2.1, with little modification. On the other hand, formula (2.8) has already been proved as [37, Proposition 2.12] and $[43,(4.4)]$, and the non-scale-invariant estimate (2.9) is easy. Hence we omit the proof of Lemma 2.2. The following tools will be used to prove Lemma 2.1.

Lemma 2.3. (Strichartz estimate on $\left.\mathbb{T}^{4}[4,43]\right)$. For $p>3$,

$$
\left\|P_{\leq M} u\right\|_{L_{t, x}^{p}} \lesssim M^{2-\frac{6}{p}}\|u\|_{Y^{0}} .
$$

Corollary 2.4. (Strichartz estimates on $\mathbb{T}^{4}$ with noncentred frequency localisation). Let $M$ be a dyadic value and let $Q$ be a (possibly) noncentred $M$-cube in Fourier space,

$$
Q=\left\{\xi_{0}+\eta:|\eta| \leq M\right\}
$$

Let $P_{Q}$ be the corresponding Littlewood-Paley projection. Then by Galilean invariance, we have

$$
\left\|P_{Q} u\right\|_{L_{t, x}^{p}} \lesssim M^{2-\frac{6}{p}}\left\|P_{Q} u\right\|_{Y^{0}} \quad p>3
$$

The net effect of this observation is that we pay a factor of only $M^{2-\frac{6}{p}}$ when applying formula (2.12).

Proof. Such a fact is well known and widely used. Readers interested in a version of the proof can see [24, Corollary 5.18].

\subsection{Proof of Lemma 2.1}

We first present the proof of the sharp estimate (2.4), then that of formula (2.5). 


\subsubsection{Proof of formula (2.4)}

Let $I$ denote the integral in formula (2.4). Decompose the four factors into Littlewood-Paley pieces so that

$$
I=\sum_{M_{1}, M_{2}, M_{3}, M} I_{M_{1}, M_{2}, M_{3}, M}
$$

where

$$
I_{M_{1}, M_{2}, M_{3}, M}=\iint_{x, t} u_{1, M_{1}} u_{2, M_{2}} u_{3, M_{3}} g_{M} d x d t
$$

with $u_{j, M_{j}}=P_{M_{j}} u_{j}$ and $g_{M}=P_{M} g$. As $M_{2}, M_{3}$ and $M$ are symmetric, it suffices to take care of the $M_{1} \sim M_{2} \geq M_{3} \geq M$ case. Decompose the $M_{1}$ and $M_{2}$ dyadic spaces into $M_{3}$-size cubes; then

$$
\begin{aligned}
I_{1 A} & \lesssim \sum_{\substack{M_{1}, M_{2}, M_{3}, M \\
M_{1} \sim M_{2} \geq M_{3} \geq M}} \sum_{Q}\left\|P_{Q} u_{1, M_{1}} P_{Q_{c}} u_{2, M_{2}} u_{3, M_{3}} g_{M}\right\|_{L_{t, x}^{1}} \\
& \lesssim \sum_{\substack{M_{1}, M_{2}, M_{3}, M \\
M_{1} \sim M_{2} \geq M_{3} \geq M}} \sum_{Q}\left\|P_{Q} u_{1, M_{1}}\right\|_{L_{t, x}^{\frac{10}{3}}}\left\|P_{Q_{c}} u_{2, M_{2}}\right\|_{L_{t, x}^{\frac{10}{3}}}\left\|u_{3, M_{3}}\right\|_{L_{t, x}^{\frac{10}{3}}}\left\|g_{M}\right\|_{L_{t, x}^{10}} .
\end{aligned}
$$

Using formulas (2.12) and (2.13),

$$
\begin{aligned}
& \lesssim \sum_{\substack{M_{1}, M_{2}, M_{3}, M \\
M_{1} \sim M_{2} \geq M_{3} \geq M}} \sum_{Q} M_{3}^{\frac{2}{5}}\left\|P_{Q^{2}} u_{1, M_{1}}\right\|_{Y^{0}}\left\|u_{3, M_{3}}\right\|_{Y^{0}} M_{3}^{\frac{1}{5}}\left\|P_{Q_{c}} u_{2, M_{2}}\right\|_{Y^{0}} M^{\frac{7}{5}}\left\|g_{M}\right\|_{Y^{0}} \\
& \lesssim \sum_{\substack{M_{1}, M_{2}, M_{3}, M \\
M_{1} \sim M_{2} \geq M_{3} \geq M}} M_{3}^{\frac{3}{5}} M^{\frac{7}{5}}\left\|g_{M}\right\|_{Y^{0}}\left\|u_{3, M_{3}}\right\|_{Y^{0}} \sum_{Q}\left\|P_{Q} u_{1, M_{1}}\right\|_{Y^{0}}\left\|P_{Q_{c}} u_{2, M_{2}}\right\|_{Y^{0}} .
\end{aligned}
$$

Applying Cauchy-Schwarz to sum in $Q$, we have

$$
\begin{aligned}
& \lesssim \sum_{\substack{M_{1}, M_{2}, M_{3}, M \\
M_{1} \sim M_{2} \geq M_{3} \geq M}} M_{3}^{\frac{3}{5}} M^{\frac{7}{5}}\left\|u_{1, M_{1}}\right\|_{Y^{0}}\left\|u_{2, M_{2}}\right\|_{Y^{0}}\left\|u_{3, M_{3}}\right\|_{Y^{0}}\left\|g_{M}\right\|_{Y^{0}} \\
& \lesssim \sum_{\substack{M_{1}, M_{2} \\
M_{1} \sim M_{2}}} M_{2} M_{1}^{-1}\left\|u_{1, M_{1}}\right\|_{Y^{-1}}\left\|u_{2, M_{2}}\right\|_{Y^{1}} \sum_{\substack{M_{3}, M \\
M_{1} \sim M_{2} \geq M_{3} \geq M}} M_{3}^{-\frac{2}{5}} M^{\frac{2}{5}}\left\|u_{3, M_{3}}\right\|_{Y^{1}}\left\|g_{M}\right\|_{Y^{1}} .
\end{aligned}
$$

We are done, by Schur's test.

\subsubsection{Proof of formula (2.5)}

We reuse the setup from the proof of formula (2.4). However, due to the symmetry assumption $M_{1} \geq$ $M_{2} \geq M_{3}$ on the frequencies in the proof of formula (2.4), we cannot simply assume that $P_{\leq M_{0}}$ lands on $u_{2}$. The worst-gain (least-gain) case here would be that $u_{1}$ is still put in $Y^{-1}$ and $P_{\leq M_{0}}$ is applied to $u_{3}$. Thus we will prove estimate (2.5) subject to the extra localisation that $P_{\leq M_{0}}$ be applied on $u_{3}$. By symmetry in $M_{2}$ and $M$, it suffices to take care of two cases: A, $M_{1} \sim M_{2} \geq M_{3} \geq M$, and B, $M_{1} \sim M_{2} \geq M \geq M_{3}$. We will get a $T^{\frac{1}{4}} M_{0}^{\frac{3}{5}}$ in case A and a $T^{\frac{1}{7}} M_{0}^{\frac{3}{7}}$ in case B. Since formula (2.5) is nowhere near optimal, and we just need it to hold with some powers of $T$ and $M_{0}$, there is no need to match these powers or pursue the best power in these cases. 
Case A: $\boldsymbol{M}_{1} \sim \boldsymbol{M}_{2} \geq \boldsymbol{M}_{3} \geq \boldsymbol{M}$.

Decompose the $M_{1}$ and $M_{2}$ dyadic spaces into $M_{3}$-size cubes:

$$
\begin{aligned}
I_{M_{1}, M_{2}, M_{3}, M} & \leq \sum_{Q}\left\|P_{Q} u_{1, M_{1}} P_{Q_{c}} u_{2, M_{2}}\left(P_{\leq M_{0}} u_{3, M_{3}}\right) g_{M}\right\|_{L_{t, x}^{1}} \\
& \leq \sum_{Q}\left\|P_{Q} u_{1, M_{1}} g_{M}\right\|_{L_{t, x}^{2}}\left\|P_{Q_{C}} u_{2, M_{2}}\right\|_{L_{t, x}^{4}}\left\|P_{\leq M_{0}} u_{3, M_{3}}\right\|_{L_{t, x}^{4}},
\end{aligned}
$$

where

$$
\left\|P_{\leq M_{0}} u_{3, M_{3}}\right\|_{L_{t, x}^{4}} \leq T^{\frac{1}{4}} M_{0}^{\frac{3}{5}} M_{3}^{\frac{2}{5}}\left\|P_{\leq M_{0}} u_{3, M_{3}}\right\|_{L_{t}^{\infty} L_{x}^{2}} \lesssim T^{\frac{1}{4}} M_{0}^{\frac{3}{5}} M_{3}^{\frac{2}{5}}\left\|P_{\leq M_{0}} u_{3, M_{3}}\right\|_{Y^{0}} .
$$

Using formulas (2.12) and (2.13),

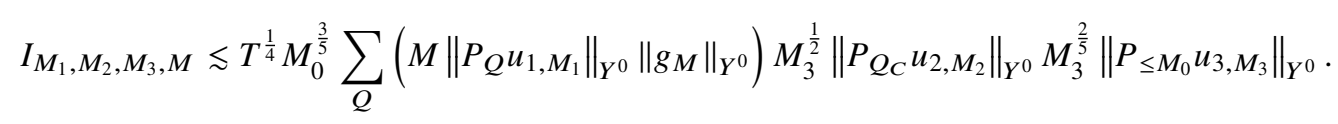

Note that we actually used a bilinear estimate for the first factor, but did not record or use the bilinear gain factor. Using Cauchy-Schwarz to sum in $Q$, we have

$$
I_{M_{1}, M_{2}, M_{3}, M} \lesssim T^{\frac{1}{4}} M_{0}^{\frac{3}{5}}\left\|u_{1, M_{1}}\right\|_{Y^{0}}\left\|g_{M}\right\|_{Y^{1}}\left\|u_{2, M_{2}}\right\|_{Y^{0}} M_{3}^{\frac{9}{10}}\left\|P_{\leq M_{0}} u_{3, M_{3}}\right\|_{Y^{0}} .
$$

Thus, summing in $M$ nonoptimally gives

$$
\begin{aligned}
I_{1 A} & \lesssim T^{\frac{1}{4}} M_{0}^{\frac{3}{5}}\|g\|_{Y^{1}} \sum_{\substack{M_{1}, M_{2}, M_{3} \\
M_{1} \sim M_{2} \geq M_{3}}}\left\|u_{1, M_{1}}\right\|_{Y^{0}}\left\|u_{2, M_{2}}\right\|_{Y^{0}} M_{3}^{\frac{9}{10}} \log M_{3}\left\|P_{\leq M_{0}} u_{3, M_{3}}\right\|_{Y^{0}} \\
& \lesssim T^{\frac{1}{4}} M_{0}^{\frac{3}{5}}\|g\|_{Y^{1}} \sum_{\substack{M_{1}, M_{2}, M_{3} \\
M_{1} \sim M_{2} \geq M_{3}}}\left\|u_{1, M_{1}}\right\|_{Y^{0}}\left\|u_{2, M_{2}}\right\|_{Y^{0}} \frac{M_{3}^{\frac{9}{10}} \log M_{3}}{M_{3}}\left\|P_{\leq M_{0}} u_{3, M_{3}}\right\|_{Y^{1}} .
\end{aligned}
$$

Again, summing in $M_{3}$ nonoptimally and swapping a derivative between $u_{1}$ and $u_{2}$ give

$$
\begin{aligned}
I_{1 A} & \lesssim T^{\frac{1}{4}} M_{0}^{\frac{3}{5}}\|g\|_{Y^{1}}\left\|P_{\leq M_{0}} u_{3}\right\|_{Y^{1}} \sum_{\substack{M_{1}, M_{2} \\
M_{1} \sim M_{2}}}\left\|u_{1, M_{1}}\right\|_{Y^{-1}}\left\|u_{2, M_{2}}\right\|_{Y^{1}} \\
& \lesssim T^{\frac{1}{4}} M_{0}^{\frac{3}{5}}\left\|u_{1}\right\|_{Y^{-1}}\left\|u_{2}\right\|_{Y^{1}}\left\|P_{\leq M_{0}} u_{3}\right\|_{Y^{1}}\|g\|_{Y^{1}}
\end{aligned}
$$

Case B: $\boldsymbol{M}_{\mathbf{1}} \sim \boldsymbol{M}_{\mathbf{2}} \geq \boldsymbol{M} \geq \boldsymbol{M}_{\mathbf{3}}$.

Sum up $M_{3}$ first. We then consider

$$
I_{M_{1}, M_{2}, M}=\iint_{x, t} u_{1, M_{1}} u_{2, M_{2}}\left(P_{\leq M} P_{\leq M_{0}} u_{3}\right) g_{M} d x d t .
$$

Decompose the $M_{1}$ and $M_{2}$ dyadic spaces into $M$-size cubes:

$$
\begin{aligned}
I_{M_{1}, M_{2}, M} & \leq \sum_{Q}\left\|P_{Q} u_{1, M_{1}} P_{Q_{c}} u_{2, M_{2}}\left(P_{\leq M} P_{\leq M_{0}} u_{3}\right) g_{M}\right\|_{L_{t, x}^{1}} \\
& \leq \sum_{Q}\left\|P_{Q} u_{1, M_{1}}\right\|_{L_{t, x}^{\frac{7}{2}}}\left\|P_{Q_{C}} u_{2, M_{2}}\right\|_{L_{t, x}^{\frac{7}{2}}}\left\|P_{\leq M} P_{\leq M_{0}} u_{3}\right\|_{L_{t, x}^{7}}\left\|g_{M}\right\|_{L_{t, x}^{\frac{7}{2}}},
\end{aligned}
$$


where

$$
\begin{aligned}
\left\|P_{\leq M} P_{\leq M_{0}} u_{3}\right\|_{L_{t, x}^{7}} & \leq T^{\frac{1}{7}}\left\|P_{\leq M} P_{\leq M_{0}} u_{3}\right\|_{L_{t}^{\infty} L_{x}^{7}} \\
& \lesssim T^{\frac{1}{7}} M_{0}^{\frac{3}{7}}\left\|P_{\leq M} P_{\leq M_{0}} u_{3}\right\|_{L_{t}^{\infty} H_{x}^{1}} \\
& \lesssim T^{\frac{1}{7}} M_{0}^{\frac{3}{7}}\left\|P_{\leq M} P_{\leq M_{0}} u_{3}\right\|_{Y^{1}} .
\end{aligned}
$$

Applying formulas (2.12) and (2.13),

$$
I_{M_{1}, M_{2}, M} \lesssim T^{\frac{1}{7}} M_{0}^{\frac{3}{7}}\left\|P_{\leq M} P_{\leq M_{0}} u_{3}\right\|_{Y^{1}} \sum_{Q} M^{\frac{2}{7}}\left\|P_{Q} u_{1, M_{1}}\right\|_{Y^{0}} M^{\frac{2}{7}}\left\|P_{Q_{C}} u_{2, M_{2}}\right\|_{Y^{0}} M^{\frac{2}{7}}\left\|g_{M}\right\|_{Y^{0}}
$$

Applying Cauchy-Schwarz to sum in $Q$, we have

$$
I_{M_{1}, M_{2}, M} \lesssim T^{\frac{1}{7}} M_{0}^{\frac{3}{7}}\left\|P_{\leq M} P_{\leq M_{0}} u_{3}\right\|_{Y^{1}} M^{-\frac{1}{7}}\left\|u_{1, M_{1}}\right\|_{Y^{0}}\left\|u_{2, M_{2}}\right\|_{Y^{0}}\left\|g_{M}\right\|_{Y^{1}}
$$

Thus, swapping a derivative between $u_{1}$ and $u_{2}$ gives

$$
I_{1 B} \lesssim T^{\frac{1}{7}} M_{0}^{\frac{3}{7}}\left\|P_{\leq M_{0}} u_{3}\right\|_{Y^{1}} \sum_{\substack{M_{1}, M_{2}, M \\ M_{1} \sim M_{2} \geq M}} M^{-\frac{1}{7}}\left\|u_{1, M_{1}}\right\|_{Y^{-1}}\left\|u_{2, M_{2}}\right\|_{Y^{1}}\left\|g_{M}\right\|_{Y^{1}}
$$

Burning that $\frac{1}{7}$-derivative to sum in $M$ and then applying Cauchy-Schwarz in $M_{1}$, we have

$$
I_{1 B} \lesssim T^{\frac{1}{7}} M_{0}^{\frac{3}{7}}\left\|P_{\leq M_{0}} u_{3}\right\|_{Y^{1}}\left\|u_{1}\right\|_{Y^{-1}}\left\|u_{2}\right\|_{Y^{1}}\|g\|_{Y^{1}}
$$

as needed.

\section{Uniqueness for GP hierarchy (1.2) and the proof of Theorem 1.1 - Setup}

Theorem 3.1. Let $\Gamma=\left\{\gamma^{(k)}\right\} \in \oplus_{k \geq 1} C\left(\left[0, T_{0}\right], \mathcal{L}_{k}^{1}\right)$ be a solution to equation (1.2) in $\left[0, T_{0}\right]$, in the sense that

(a) $\Gamma$ is admissible in the sense of Definition 3.4 and

(b) $\Gamma$ satisfies the kinetic energy condition that $\exists C_{0}>0$ such that

$$
\sup _{t \in\left[0, T_{0}\right]} \operatorname{Tr}\left(\prod_{j=1}^{k}\left\langle\nabla_{x_{j}}\right\rangle\right) \gamma^{(k)}(t)\left(\prod_{j=1}^{k}\left\langle\nabla_{x_{j}}\right\rangle\right) \leq C_{0}^{2 k}
$$

Then there is a threshold $\eta\left(C_{0}\right)>0$ such that the solution is unique in $\left[0, T_{0}\right]$, provided

$$
\sup _{t \in\left[0, T_{0}\right]} \operatorname{Tr}\left(\prod_{j=1}^{k} P_{>M}^{j}\left\langle\nabla_{x_{j}}\right\rangle\right) \gamma^{(k)}(t)\left(\prod_{j=1}^{k} P_{>M}^{j}\left\langle\nabla_{x_{j}}\right\rangle\right) \leq \eta^{2 k}
$$

for some frequency $M$. Our proof shows that $\eta\left(C_{0}\right)$ can be $\left(100 C C_{0}\right)^{-2}$, with $C$ being a universal constant depending on the $U$-V estimate constants and the Sobolev constants. The frequency threshold $M$ is allowed to depend on $\gamma^{(k)}$ (the particular solution under consideration) but must apply uniformly on $\left[0, T_{0}\right]$. 
Here, we have intentionally stated Theorem 3.1 before writing out the definition of admissibility (Definition 3.4) to bring up readers' attention. For the purpose of only proving Theorem 1.1, in fact, Definition 3.4 and its companion, the quantum de Finetti theorem (Theorem 3.5), are not necessary. One could just apply the proof of Theorem 3.1 to the special case

$$
\gamma^{(k)}(t) \equiv \int_{L^{2}\left(\mathbb{T}^{4}\right)}|\phi\rangle\left\langle\left.\phi\right|^{\otimes k} d \mu_{t}(\phi) \equiv \prod_{j=1}^{k} u_{1}\left(t, x_{j}\right) \bar{u}_{1}\left(t, x_{j}^{\prime}\right)-\prod_{j=1}^{k} u_{2}\left(t, x_{j}\right) \bar{u}_{2}\left(t, x_{j}^{\prime}\right),\right.
$$

where $u_{1}$ and $u_{2}$ are two solutions to equation (1.1) and $\mu_{t}$ is the signed measure $\delta_{u_{1}}-\delta_{u_{2}}$ on $L^{2}\left(\mathbb{T}^{4}\right)$, to get that the difference is zero for all $k$ and to obtain a uniqueness theorem which is solely about solutions to equation (1.1). This is sufficient to conclude Theorem 1.1. Readers unfamiliar with Theorem 3.5 could first skip Definition 3.4 and Theorem 3.5, put equation (3.1) in the place of equation (3.6), get to know how the GP hierarchy is involved and then come back to Definition 3.4 and Theorem 3.5. Once one understands the role of the GP hierarchy in the proof, it is easy to see that due to Theorem 3.5, the more general theorem (Theorem 3.1) costs nothing more, and the origin of the current scheme of proving NLS uniqueness using GP hierarchies is indeed Theorem 3.5, as mentioned in the introduction. Theorem 3.1 also implies the following corollary:

Corollary 3.2. Given an intial datum $u_{0} \in H^{1}\left(\mathbb{T}^{4}\right)$, there is at most one $C\left(\left[0, T_{0}\right], H_{x, \text { weak }}^{1}\right)$ solution u to equation (1.1) on $\mathbb{T}^{4}$ satisfying the following two properties:

(1) There is a $C_{0}>0$ such that

$$
\sup _{t \in\left[0, T_{0}\right]}\|u(t)\|_{H^{1}} \leq C_{0} .
$$

(2) There is some frequency $M$ such that

$$
\sup _{t \in\left[0, T_{0}\right]}\left\|\nabla P_{\geq M} u(t)\right\|_{L_{x}^{2}} \leq \eta,
$$

for the threshold $\eta\left(C_{0}\right)>0$ concluded in Theorem 3.1.

The known $C\left(\left[0, T_{0}\right], H_{x \text {,weak }}^{1}\right)$ blowup solutions do not satisfy formula (3.2), so Corollary 3.2 is an unclassified uniqueness theorem. It seems to be stronger than the unconditional uniqueness theorem (Theorem 1.1), as it concludes uniqueness in a larger class of solutions. We wonder if there could be a more detailed classification regarding the term 'unconditional uniqueness' at the critical regularity.

Theorem 1.1 follows from Theorem 3.1 and the following lemma:

Lemma 3.3. $u$ is a $C_{\left[0, T_{0}\right]}^{0} H_{x}^{1} \cap \dot{C}_{\left[0, T_{0}\right]}^{1} H_{x}^{-1}$ solution of equation (1.1) if and only if it is a $C_{\left[0, T_{0}\right]}^{0} H_{x \text {, weak }}^{1} \cap$ $\dot{C}_{\left[0, T_{0}\right]}^{1} H_{x, \text { weak }}^{-1}$ solution and satisfies uniform-in-time frequency localisation - that is, for each $\varepsilon>0$ there exists $M(\varepsilon)$ such that

$$
\left\|\nabla P_{\geq M(\varepsilon)} u\right\|_{L_{\left[0, T_{0}\right]}^{\infty} L_{x}^{2}} \leq \varepsilon .
$$

Proof. This proof is postponed to Section 3.1. We remark that formula (3.3) implies formula (3.2), but the converse is not true. That is, Corollary 3.2 implies Theorem 1.1, the unconditional uniqueness theorem, but the type of uniqueness concluded in Corollary 3.2 and Theorem 3.1 is unclassified. ${ }^{15}$

${ }^{15}$ The proof of Lemma 3.3 uses only compactness and is much simpler than that of [24, Theorem A.2]. 
Before starting the proof of Theorem 3.1, we note that although it seems that Theorem 3.1 concludes Theorem 1.1 only up to a time-dependent $e^{i \theta(t)}$ phase, $\theta(t)$ is actually 0 (see [35, p. 12] and [26, Lemma A.1]). Thus, we are left to prove Theorem 3.1.

We set up some notations first. We rewrite equation (1.2) in Duhamel form:

$$
\gamma^{(k)}\left(t_{k}\right)=U^{(k)}\left(t_{k}\right) \gamma_{0}^{(k)} \mp i \int_{0}^{t_{k}} U^{(k)}\left(t_{k}-t_{k+1}\right) B^{(k+1)}\left(\gamma^{(k+1)}\left(t_{k+1}\right)\right) d t_{k+1},
$$

where $U^{(k)}(t)=\prod_{j=1}^{k} e^{i t\left(\Delta_{x_{j}}-\Delta_{x_{j}^{\prime}}\right)}$ and

$$
\begin{aligned}
B^{(k+1)}\left(\gamma^{(k+1)}\right) & \equiv \sum_{j=1}^{k} B_{j, k+1}\left(\gamma^{(k+1)}\right) \\
& \equiv \sum_{j=1}^{k}\left(B_{j, k+1}^{+}-B_{j, k+1}^{-}\right)\left(\gamma^{(k+1)}\right) \\
& \equiv \sum_{j=1}^{k} \operatorname{Tr}_{k+1} \delta\left(x_{j}-x_{k+1}\right) \gamma^{(k+1)}-\gamma^{(k+1)} \delta\left(x_{j}-x_{k+1}\right)
\end{aligned}
$$

Here, products are interpreted as the compositions of operators. For example, in kernels,

$$
\left(\operatorname{Tr}_{k+1} \delta\left(x_{1}-x_{k+1}\right) \gamma^{(k+1)}\right)\left(\mathbf{x}_{k}, \mathbf{x}_{k}^{\prime}\right)=\int \delta\left(x_{1}-x_{k+1}\right) \gamma^{(k+1)}\left(\mathbf{x}_{k}, x_{k+1} ; \mathbf{x}_{k}^{\prime}, x_{k+1}\right) d x_{k+1} .
$$

We will prove that if $\Gamma_{1}=\left\{\gamma_{1}^{(k)}\right\}$ and $\Gamma_{2}=\left\{\gamma_{2}^{(k)}\right\}$ are two solutions to equation (3.4), subject to the same initial datum and Theorem 3.1(a) and (b), then $\Gamma=\left\{\gamma^{(k)}=\gamma_{1}^{(k)}-\gamma_{2}^{(k)}\right\}$ is identically zero. Note that because equation (3.4) is linear, $\Gamma$ is a solution to equation (3.4). We will start using a representation of $\Gamma$ given by the quantum de Finetti theorem (Theorem 3.5). To this end, we define admissibility:

Definition 3.4. ([6]). A nonnegative trace class symmetric operators sequence $\Gamma=\left\{\gamma^{(k)}\right\} \in$ $\oplus_{k \geq 1} C\left([0, T], \mathcal{L}_{k}^{1}\right)$, is called admissible if for all $k$, one has

$$
\operatorname{Tr} \gamma^{(k)}=1, \quad \gamma^{(k)}=\operatorname{Tr}_{k+1} \gamma^{(k+1)} .
$$

Here, a trace class operator is called symmetry if, written in kernel form,

$$
\begin{aligned}
\gamma^{(k)}\left(\mathbf{x}_{k} ; \mathbf{x}_{k}^{\prime}\right) & =\overline{\gamma^{(k)}\left(\mathbf{x}_{k}^{\prime} ; \mathbf{x}_{k}\right)} \\
\gamma^{(k)}\left(x_{1}, \ldots x_{k} ; x_{1}^{\prime}, \ldots, x_{k}^{\prime}\right) & =\gamma^{(k)}\left(x_{\sigma(1)}, \ldots, x_{\sigma(k)} ; x_{\sigma(1)}^{\prime}, \ldots, x_{\sigma(k)}^{\prime}\right),
\end{aligned}
$$

for all $\sigma \in S_{k}$, the permutation group of $k$ elements.

Theorem 3.5. (quantum de Finetti theorem [6, 51]). Under assumption (a), there exists a probability measure $d \mu_{t}(\phi)$ supported on the unit sphere of $L^{2}\left(\mathbb{T}^{4}\right)$ such that

$$
\gamma^{(k)}(t)=\int|\phi\rangle\left\langle\left.\phi\right|^{\otimes k} d \mu_{t}(\phi) .\right.
$$

By Theorem 3.5, there exist $d \mu_{1, t}$ and $d \mu_{2, t}$ representing the two solutions $\Gamma_{1}$ and $\Gamma_{2}$. The same Chebyshev argument as in [6, Lemma 4.5] turns the assumptions in Theorem 3.1 into the property that 
$d \mu_{j, t}$ is supported in the set

$$
S=\left\{\phi \in \mathbb{S}\left(L^{2}\left(\mathbb{T}^{4}\right)\right):\left\|P_{>M}\langle\nabla\rangle \phi\right\|_{L^{2}} \leq \varepsilon\right\} \cap\left\{\phi \in \mathbb{S}\left(L^{2}\left(\mathbb{T}^{4}\right)\right):\|\phi\|_{H^{1}} \leq C_{0}\right\} .
$$

That is, letting the signed measure $d \mu_{t}=d \mu_{1, t}-d \mu_{2, t}$, we have

$$
\gamma^{(k)}\left(t_{k}\right)=\left(\gamma_{1}^{(k)}-\gamma_{2}^{(k)}\right)\left(t_{k}\right)=\int|\phi\rangle\left\langle\left.\phi\right|^{\otimes k} d \mu_{t_{k}}(\phi)\right.
$$

and $d \mu_{t_{k}}$ is supported in the set $S$ defined in equation (3.5).

So our task of establishing Theorem 3.1 is now transformed into proving that the solution is zero if the solution takes the form of equation (3.6) and is subject to zero initial datum. It suffices to prove $\gamma^{(1)}=0$, as the proof is the same for the general $k$ case. The proof involves coupling equation (3.4) multiple times. To this end, we plug in zero initial datum, set the $\mp i$ in equation (3.4) to 1 so that we do not need track its power (because it acts as a 1 in the estimates for our purpose) and rewrite equation (3.4) as

$$
\gamma^{(k)}\left(t_{k}\right)=\int_{0}^{t_{k}} U^{(k)}\left(t_{k}-t_{k+1}\right) B^{(k+1)}\left(\gamma^{(k+1)}\left(t_{k+1}\right)\right) d t_{k+1} .
$$

Define

$$
J^{(k+1)}\left(f^{(k+1)}\right)\left(t_{1}, \underline{t}_{k+1}\right)=U^{(1)}\left(t_{1}-t_{2}\right) B^{(2)} U^{(2)}\left(t_{2}-t_{3}\right) B^{(3)} \cdots U^{(k)}\left(t_{k}-t_{k+1}\right) B^{(k+1)} f^{(k+1)}\left(t_{k+1}\right),
$$

with $\underline{t}_{k+1}=\left(t_{2}, t_{3}, \ldots, t_{k}, t_{k+1}\right)$. We can then write

$$
\gamma^{(1)}\left(t_{1}\right)=\int_{0}^{t_{1}} \int_{0}^{t_{2}} \cdots \int_{0}^{t_{k}} J^{(k+1)}\left(\gamma^{(k+1)}\right)\left(t_{1}, \underline{t}_{k+1}\right) d \underline{t}_{k+1},
$$

after iterating equation (3.7) $k$ times. To estimate $\gamma^{(1)}$, we first use the KM board game ${ }^{16}$ to reduce the number of summands inside $\gamma^{(1)}$, which is $k ! 2^{k}$ at the moment, by combining them.

Lemma 3.6. (Klainerman-Machedon board game [47]). One can express

$$
\int_{0}^{t_{1}} \int_{0}^{t_{2}} \cdots \int_{0}^{t_{k+1}} J^{(k+1)}\left(f^{(k+1)}\right)\left(t_{1}, \underline{t}_{k+1}\right) d \underline{t}_{k+1}
$$

as a sum of at most $4^{k}$ terms of the form

$$
\int_{D_{m}} J_{\mu_{m}}^{(k+1)}\left(f^{(k+1)}\right)\left(t_{1}, \underline{t}_{k+1}\right) d \underline{t}_{k+1}
$$

or in other words,

$$
\int_{0}^{t_{1}} \int_{0}^{t_{2}} \cdots \int_{0}^{t_{k+1}} J^{(k+1)}\left(f^{(k+1)}\right)\left(t_{1}, \underline{t}_{k+1}\right) d \underline{t}_{k+1}=\sum_{m} \int_{D_{m}} J_{\mu_{m}}^{(k+1)}\left(f^{(k+1)}\right)\left(t_{1}, \underline{t}_{k+1}\right) d \underline{t}_{-k+1} .
$$

Here, $D_{m}$ is a subset of $\left[0, t_{1}\right]^{k}$, depending on $\mu_{m} ;\left\{\mu_{m}\right\}$ are a set of maps from $\{2, \ldots, k+1\}$ to $\{1, \ldots, k\}$ satisfying $\mu_{m}(2)=1$ and $\mu_{m}(l)<l$ for all $l$, and

$$
\begin{aligned}
J_{\mu_{m}}^{(k+1)}\left(f^{(k+1)}\right)\left(t_{1}, \underline{t}_{k+1}\right) & =U^{(1)}\left(t_{1}-t_{2}\right) B_{1,2} U^{(2)}\left(t_{2}-t_{3}\right) B_{\mu_{m}(3), 3} \cdots \\
& \cdots U^{(k)}\left(t_{k}-t_{k+1}\right) B_{\mu_{m}(k+1), k+1}\left(f^{(k+1)}\right)\left(t_{1}\right) .
\end{aligned}
$$

${ }^{16}$ As mentioned before, we actually need an extended KM board game, which we devise in $\S 4$. 
Using Lemma 3.6, to estimate $\gamma^{(1)}$ it suffices to deal with a summand in the right-hand side of equation (3.8),

$$
\int_{D_{m}} J_{\mu_{m}}^{(k+1)}\left(\gamma^{(k+1)}\right)\left(t_{1}, \underline{t}_{k+1}\right) d \underline{t}_{k+1}
$$

at the expense of a $4^{k}$. Since $B_{j, k+1}=B_{j, k+1}^{+}-B_{j, k+1}^{-}, J_{\mu_{m}}^{(k+1)}\left(\gamma^{(k+1)}\right)$ is but another sum. Thus, by paying an extra $2^{k}$, we can just estimate a typical term

$$
\int_{D_{m}} J_{\mu_{m}, \mathrm{sgn}}^{(k+1)}\left(\gamma^{(k+1)}\right)\left(t_{1}, \underline{t}_{k+1}\right) d \underline{t}_{k+1}
$$

where

$$
\begin{array}{r}
J_{\mu_{m}, \operatorname{sgn}}^{(k+1)}\left(f^{(k+1)}\right)\left(t_{1}, \underline{t}_{k+1}\right)= \\
U^{(1)}\left(t_{1}-t_{2}\right) B_{1,2}^{\operatorname{sgn}(2)} U^{(2)}\left(t_{2}-t_{3}\right) B_{\mu_{m}(3), 3}^{\operatorname{sgn}(3)} \cdots \\
\cdots U^{(k)}\left(t_{k}-t_{k+1}\right) B_{\mu_{m}(k+1), k+1}^{\operatorname{sgn}(k+1)}\left(f^{(k+1)}\right)\left(t_{k+1}\right)
\end{array}
$$

with sgn meaning the signature array $(\operatorname{sgn}(2), \ldots, \operatorname{sgn}(k+1))$ and $B_{j, k+1}^{\operatorname{sgn}(k+1)}$ standing for $B_{j, k+1}^{+}$or $B_{j, k+1}^{-}$, depending on the sign of the $(k+1)$-th signature element. The estimate of expression (3.9) is given by the following proposition:

\section{Proposition 3.7.}

$$
\left\|\left\langle\nabla_{x_{1}}\right\rangle^{-1}\left\langle\nabla_{x_{1}^{\prime}}\right\rangle^{-1} \int_{D_{m}} J_{\mu_{m}, \text { sgn }}^{(k+1)}\left(\gamma^{(k+1)}\right)\left(t_{1}, \underline{t}_{k+1}\right) d \underline{t}_{-k+1}\right\|_{L_{t_{1}}^{\infty} L_{x_{1}, x_{1}^{\prime}}^{2}} \leq 2 T C_{0}^{2}\left(C C_{0}^{3} T^{\frac{1}{7}} M_{0}^{\frac{3}{5}}+C C_{0}^{2} \varepsilon\right)^{\frac{2}{3} k}
$$

Proof. See Section 5.

Once Proposition 3.7 is proved, Theorem 3.1 then follows. In fact,

$$
\begin{aligned}
\|\left\langle\nabla_{x_{1}}^{-1}\right\rangle & \left\langle\nabla_{x_{1}^{\prime}}\right\rangle^{-1} \gamma^{(1)}\left(t_{1}\right) \|_{L_{t_{1}}^{\infty} L_{x_{1}, x_{1}^{\prime}}^{2}} \\
& \leq 4^{k}\left\|\left\langle\nabla_{x_{1}}\right\rangle^{-1}\left\langle\nabla_{x_{1}^{\prime}}\right\rangle^{-1} \int_{D_{m}} J_{\mu_{m}}^{(k+1)}\left(\gamma^{(k+1)}\right)\left(t_{1}, \underline{t}_{k+1}\right) d \underline{t}_{k+1}\right\|_{L_{t_{1}}^{\infty} L_{x_{1}, x_{1}^{\prime}}^{2}} \\
& \leq 8^{k}\left\|\left\langle\nabla_{x_{1}}\right\rangle^{-1}\left\langle\nabla_{x_{1}^{\prime}}\right\rangle^{-1} \int_{D_{m}} J_{\mu_{m}, \operatorname{sgn}}^{(k+1)}\left(\gamma^{(k+1)}\right)\left(t_{1}, \underline{t}_{k+1}\right) d \underline{t}_{k+1}\right\|_{L_{t_{1}}^{\infty} L_{x_{1}, x_{1}^{\prime}}^{2}} \\
& \leq 2 T C_{0}^{2}\left(C C_{0}^{3} T^{\frac{1}{7}} M_{0}^{\frac{3}{5}}+C C_{0}^{2} \varepsilon\right)^{\frac{2}{3} k} .
\end{aligned}
$$

Select $\varepsilon$ small enough (the threshold $\eta$ is also determined here) so that $C C_{0}^{2} \varepsilon<\frac{1}{4}$ and then select $T$ small enough so that $C C_{0}^{3} T^{\frac{1}{7}} M_{0}^{\frac{3}{5}}<\frac{1}{4}$. We then have

$$
\left\|\left\langle\nabla_{x_{1}}\right\rangle^{-1}\left\langle\nabla_{x_{1}^{\prime}}\right\rangle^{-1} \gamma^{(1)}\left(t_{1}\right)\right\|_{L_{t_{1}}^{\infty} L_{x_{1}, x_{1}^{\prime}}^{2}} \leq\left(\frac{1}{2}\right)^{k} \rightarrow 0 \text { as } k \rightarrow \infty .
$$

We can then bootstrap to fill the whole interval $\left[0, T_{0}\right]$, as $M$ applies uniformly on $\left[0, T_{0}\right]$. 
Before moving into the proof of Proposition 3.7, we remark that the extra $2 T$ does not imply that the estimate is critical or subcritical; this $T$ actually appears only once. Such a $T$ is due to the GP-hierarchy method instead of scaling, because the $d t_{k+1}$ time integral is not used for any Strichartz-type estimates. This one factor of $T$ appears in the other energy-critical $\mathbb{T}^{3}$ quintic case [24] as well.

\subsection{Proof of Lemma 3.3 and uniform-in-time frequency localisation for the NLS}

We provide a direct proof using the equation, even though one could look for a more abstract proof. By substituting the equation, we compute

$$
\begin{aligned}
\left|\partial_{t}\left\|\nabla P_{\leq M} u\right\|_{L_{x}^{2}}^{2}\right| & =2\left|\operatorname{Im} \int P_{\leq M} \nabla u \cdot P_{\leq M} \nabla\left(|u|^{2} u\right) d x\right| \\
& \leq 2\left\|P_{\leq M} \nabla u\right\|_{L^{4}}\left\|P_{\leq M} \nabla\left(|u|^{2} u\right)\right\|_{L^{4 / 3}} \\
& =2 M^{2}\left\|\tilde{P}_{\leq M} u\right\|_{L^{4}}\left\|\tilde{P}_{\leq M}\left(|u|^{2} u\right)\right\|_{L^{4 / 3}},
\end{aligned}
$$

where if the symbol associated to $P_{\leq M}$ is $\chi(\xi / M)$, then the symbol associated to $\tilde{P}_{\leq M}$ is $\tilde{\chi}(\xi / M)$, with $\tilde{\chi}(\xi)=\xi \chi(\xi)$. By the $L^{p} \rightarrow L^{p}$ boundedness of the Littlewood-Paley projections (see, for example, [35, Appendix]),

$$
\left|\partial_{t}\left\|\nabla P_{\leq M} u\right\|_{L_{x}^{2}}^{2}\right| \lesssim M^{2}\|u\|_{L^{4}}^{4}
$$

By Sobolev embedding,

$$
\left|\partial_{t}\left\|\nabla P_{\leq M} u\right\|_{L_{x}^{2}}^{2}\right| \lesssim M^{2}\|u\|_{H^{1}}^{4}
$$

Hence there exists $\delta^{\prime}>0$ - depending on $M,\|u\|_{L_{[0, T]}^{\infty} H^{1}}$ and $\varepsilon$ - such that for any $t_{0} \in[0, T]$, it holds that for any $t \in\left(t_{0}-\delta^{\prime}, t_{0}+\delta^{\prime}\right) \cap[0, T]$,

$$
\left|\left\|\nabla P_{\leq M} u(t)\right\|_{L_{x}^{2}}^{2}-\left\|\nabla P_{\leq M} u\left(t_{0}\right)\right\|_{L_{x}^{2}}^{2}\right| \leq \frac{1}{16} \varepsilon^{2} .
$$

On the other hand, since $u \in C_{[0, T]}^{0} H_{x}^{1}$, for each $t_{0}$ there exists $\delta^{\prime \prime}>0$ such that for any $t \in\left(t_{0}-\delta^{\prime \prime}, t_{0}+\right.$ $\left.\delta^{\prime \prime}\right) \cap[0, T]$,

$$
\left|\|\nabla u(t)\|_{L_{x}^{2}}^{2}-\left\|\nabla u\left(t_{0}\right)\right\|_{L_{x}^{2}}^{2}\right| \leq \frac{1}{16} \varepsilon^{2} .
$$

Note that $\delta^{\prime \prime}$ depends on $u$ itself (or the 'modulus of continuity' of $u$ ), unlike $\delta^{\prime}$, which depends only on $M,\|u\|_{L_{[0, T]}^{\infty} H^{1}}$ and $\varepsilon$. Now let $\delta=\min \left(\delta^{\prime}, \delta^{\prime \prime}\right)$. Then by formulas (3.11) and (3.12), we have that for any $t \in\left(t_{0}-\delta, t_{0}+\delta\right) \cap[0, T]$,

$$
\left|\left\|\nabla P_{>M} u(t)\right\|_{L_{x}^{2}}^{2}-\left\|\nabla P_{>M} u\left(t_{0}\right)\right\|_{L_{x}^{2}}^{2}\right| \leq \frac{1}{4} \varepsilon^{2} .
$$

For each $t \in[0, T]$, there exists $M_{t}$ such that

$$
\left\|\nabla P_{>M_{t}} u(t)\right\|_{L_{x}^{2}} \leq \frac{1}{2} \varepsilon
$$


By the foregoing, there exists $\delta_{t}>0$ such that on $\left(t-\delta_{t}, t+\delta_{t}\right)$, we have

$$
\left\|\nabla P_{>M_{t}} u\right\|_{L_{\left(t-\delta_{t}, t+\delta_{t}\right)}^{\infty} L_{x}^{2}} \leq \varepsilon
$$

Here, $\delta_{t}>0$ depends on $u$ and $M_{t}$. The collection of intervals $\left(t-\delta_{t}, t+\delta_{t}\right)$ as $t$ ranges over $[0, T]$ is an open cover of $[0, T]$. Let

$$
\left(t_{1}-\delta_{t_{1}}, t_{1}+\delta_{t_{1}}\right), \ldots,\left(t_{J}-\delta_{t_{J}}, t_{J}+\delta_{t_{J}}\right)
$$

be an open cover of $[0, T]$. Letting

$$
M=\max \left(M_{t_{1}}, \ldots, M_{t_{J}}\right),
$$

we have established formula (3.3).

Now conversely suppose that $u \in C_{[0, T]}^{0} H_{x \text {,weak }}^{1} \cap C_{[0, T]}^{1} H_{x \text {,weak }}^{-1}$ and satisfies formula (3.3). Then we claim that $u \in C_{[0, T]}^{0} H_{x}^{1} \cap C_{[0, T]}^{1} H_{x}^{-1}$. Let $t_{0} \in[0, T]$ be arbitrary. If $u$ is not strongly continuous at $t_{0}$, then there exist $\epsilon>0$ and a sequence $t_{k} \rightarrow t_{0}$ such that $\left\|u\left(t_{k}\right)-u\left(t_{0}\right)\right\|_{H_{x}^{1}}>2 \epsilon$. Then for each $k$, there exists $\phi_{k} \in H_{x}^{-1}$ with $\left\|\phi_{k}\right\|_{H_{x}^{-1}} \leq 1$ and

$$
\left|\left\langle u\left(t_{k}\right)-u\left(t_{0}\right), \phi_{k}\right\rangle\right|>2 \epsilon .
$$

Get $M$ as in formula (3.3). Then

$$
\left|\left\langle u\left(t_{k}\right)-u\left(t_{0}\right), P_{>M} \phi_{k}\right\rangle\right| \leq \epsilon .
$$

On the other hand, by the Rellich-Kondrachov compactness theorem, there exists a subsequence such that $P_{\leq M} \phi_{k} \rightarrow \phi$ in $H_{x}^{-1}$. This combined with the assumption that $u$ is weakly continuous implies that

$$
\left\langle u\left(t_{k}\right)-u\left(t_{0}\right), P_{\leq M} \phi_{k}\right\rangle \rightarrow 0 .
$$

But formulas (3.14) and (3.15) contradict formula (3.13). The proof that $\partial_{t} u$ is strongly continuous is similarly straightforward.

\section{An extended KM board game}

This section is divided into two main parts. First, in Section 4.1, we provide as a warm-up a more elaborated proof of the original KM board game (Lemma 3.6), which yields the previously unknown time integration limits in equation (3.8). We then prove, in Sections 4.2-4.5, an extension of Lemma 3.6 which further combines the summands inside $J^{(k+1)}\left(f^{(k+1)}\right)\left(t_{1}, \underline{t}_{k+1}\right)$ to enable the application of $U-V$ space techniques.

\subsection{A more elaborated proof of Lemma 3.6}

Let us first give a brief review of the original KM board game, which since its invention has been used in every paper involving the analysis of Gross-Pitaevskii hierarchies. Recall the notation of $\mu$ in Lemma 3.6: $\{\mu\}$ is a set of maps from $\{2, \ldots, k+1\}$ to $\{1, \ldots, k\}$ satisfying $\mu(2)=1$ and $\mu(l)<l$ for all $l$, and

$$
\begin{aligned}
J_{\mu}^{(k+1)}\left(f^{(k+1)}\right)\left(t_{1}, \underline{t}_{k+1}\right) & =U^{(1)}\left(t_{1}-t_{2}\right) B_{1,2} U^{(2)}\left(t_{2}-t_{3}\right) B_{\mu(3), 3} \cdots \\
& \cdots U^{(k)}\left(t_{k}-t_{k+1}\right) B_{\mu(k+1), k+1}\left(f^{(k+1)}\left(t_{k+1}\right)\right) .
\end{aligned}
$$


Example 1. An example of $\mu$ when $k=5$ is

$$
\begin{array}{l|lllll}
j & 2 & 3 & 4 & 5 & 6 \\
\hline \mu & 1 & 1 & 3 & 2 & 1
\end{array}
$$

If $\mu$ satisfies $\mu(j) \leq \mu(j+1)$ for $2 \leq j \leq k$ in addition to $\mu(j)<j$ for all $2 \leq j \leq k+1$, then it is in upper-echelon form ${ }^{17}$ in the terminology of [47].

Let $\mu$ be a collapsing map as already defined and $\sigma$ a permutation of $\{2, \ldots, k+1\}$. A KlainermanMachedon acceptable move, which we denote $\operatorname{KM}(j, j+1)$, is allowed when $\mu(j) \neq \mu(j+1)$ and $\mu(j+1)<j$, and is the following action: $\left(\mu^{\prime}, \sigma^{\prime}\right)=\mathrm{KM}(j, j+1)(\mu, \sigma)$ :

$$
\begin{aligned}
\mu^{\prime} & =(j, j+1) \circ \mu \circ(j, j+1), \\
\sigma^{\prime} & =(j, j+1) \circ \sigma .
\end{aligned}
$$

A key observation of Klainerman and Machedon [47] is that if $\left(\mu^{\prime}, \sigma^{\prime}\right)=\mathrm{KM}(j, j+1)(\mu, \sigma)$ and $f^{(k+1)}$ is a symmetric density, then

$$
J_{\mu^{\prime}}^{(k+1)}\left(f^{(k+1)}\right)\left(t_{1}, \sigma^{\prime-1}\left(\underline{t}_{k+1}\right)\right)=J_{\mu}^{(k+1)}\left(f^{(k+1)}\right)\left(t_{1}, \sigma^{-1}\left(\underline{t}_{k+1}\right)\right),
$$

where for $\underline{t}_{k+1}=\left(t_{2}, \ldots, t_{k+1}\right)$ we define

$$
\sigma^{-1}\left(\underline{t}_{k+1}\right)=\left(t_{\sigma^{-1}(2)}, \ldots, t_{\sigma^{-1}(k+1)}\right) .
$$

Associated to each $\mu$ and $\sigma$, we define the Duhamel integrals

$$
I\left(\mu, \sigma, f^{(k+1)}\right)\left(t_{1}\right)=\int_{t_{1} \geq t_{\sigma(2)} \geq \cdots \geq t_{\sigma(k+1)}} J_{\mu}^{(k+1)}\left(f^{(k+1)}\right)\left(t_{1}, \underline{t}_{k+1}\right) d \underline{t}_{k+1} .
$$

It follows from equation (4.1) that

$$
I\left(\mu^{\prime}, \sigma^{\prime}, f^{(k+1)}\right)=I\left(\mu, \sigma, f^{(k+1)}\right) .
$$

It is clear that we can combine Klainerman-Machedon acceptable moves as follows: If $\rho$ is a permutation of $\{2, \ldots, k+1\}$ such that it is possible to write it as a composition of transpositions

$$
\rho=\tau_{1} \circ \cdots \circ \tau_{r}
$$

for which each operator $\operatorname{KM}\left(\tau_{j}\right)$ on the right side of

$$
\mathrm{KM}(\rho) \stackrel{\text { def }}{=} \mathrm{KM}\left(\tau_{1}\right) \circ \cdots \circ \mathrm{KM}\left(\tau_{r}\right)
$$

is an acceptable action, then $\operatorname{KM}(\rho)$, defined by this composition, is acceptable as well. In this case, $\left(\mu^{\prime}, \sigma^{\prime}\right)=\operatorname{KM}(\rho)(\mu, \sigma)$ and

$$
\begin{aligned}
\mu^{\prime} & =\rho \circ \mu \circ \rho^{-1}, \\
\sigma^{\prime} & =\rho \circ \sigma,
\end{aligned}
$$

${ }^{17}$ This word makes more sense when one uses the matrix/board-game representation of $J_{\mu}^{(k+1)}\left(f^{(k+1)}\right)$ in [47]]. 
and equations (4.1) and (4.2) hold. If $\mu$ and $\mu^{\prime}$ are such that there exists $\rho$ for which $\left(\mu^{\prime}, \sigma^{\prime}\right)=$ $\operatorname{KM}(\rho)(\mu, \sigma)$, then we say that $\mu^{\prime}$ and $\mu$ are $K M$-relatable. This is an equivalence relation that partitions the set of collapsing maps into equivalence classes.

In short, one can describe the KM board game in [47] which combines the $k$ ! many terms in $J^{(k+1)}\left(f^{(k+1)}\right)$ as follows:

Algorithm 1. ([47]).

1. Convert each of the $k$ ! many $\mu_{i n}^{\prime}$ s in $J^{(k+1)}\left(f^{(k+1)}\right)$ into one of the $\leq 4^{k}$ many upper-echelon forms $\mu_{\text {out }}$ via acceptable moves, defined in the board-game argument, and at the same time produce an array $\sigma$ which changes the time integration domain from the simplex

$$
t_{1} \geq t_{2} \geq t_{3} \geq \cdots \geq t_{k+1}
$$

into the simplex

$$
t_{1} \geq t_{\sigma(2)} \geq t_{\sigma(3)} \cdots \geq t_{\sigma(k+1)} .
$$

Hence, there are $\leq 4^{k}$ classes on the right-hand side of equation (3.8).

2. For each upper-echelon form $\mu_{\text {out }}$, take a union of the time integration domains of its $\mu_{\text {in }} s$ after the acceptable moves and use it as the time integration domain for the whole class. Thus, the integration domain $D_{m}$ on the right-hand side of equation (3.8) depends on $\mu_{m}$, and we have successfully combined $k$ ! summands into $\leq 4^{k}$ summands.

The key take away in Algorithm 1 is that, although it is very much not obvious, quite a few of the summands in $J^{(k+1)}\left(f^{(k+1)}\right)$ actually have the same integrand if one switches the variable labellings in a clever way. Algorithm 1 leaves only one ambiguity - the time integration domain $D_{m}-$ which is obviously very complicated for large $k$, as it is a union of a very large number of simplexes in high dimension under the action of a proper subset of the permutation group $S_{k}$ depending on the integrand. So far, for the analysis of GP hierarchies on $\mathbb{R}^{d} / \mathbb{T}^{d}, d \leq 3$, knowing $D_{m} \subset[0,1]^{k}$ has been enough, as the related $L_{t}^{1} H^{s}$ estimates are true. $\mathbb{T}^{4}$ appears to be the first domain on which one has to know what $D_{m}$ is so that one can at least have a chance to use space-time norms like $X_{s, b}$ and $U-V$, as the related $L_{t}^{1} H^{s}$ estimates are difficult to prove and may not even be true.

It turns out that $D_{m}$ is in fact simple, as we will see. We now present a more elaborated proof of Lemma 3.6, in which $D_{m}$ is computed in a clear way. Given a $\mu$, and hence a summand inside $J^{(k+1)}\left(f^{(k+1)}\right)$, we construct a binary tree with the following algorithm:

\section{Algorithm 2.}

1. Set counter $j=2$.

2. Given $j$, find the next pair of indices $a$ and $b$ so that $a>j, b>j$,

$$
\mu(a)=\mu(j), \quad \mu(b)=j,
$$

and moreover $a$ and $b$ are the minimal indices for which the equalities hold. It is possible that there is no such a or no such $b$.

3. At the node j, put a as the left child and $b$ as the right child (if there is no a, then the $j$ node will be missing a left child, and if there is no $b$, then the $j$ node will be missing a right child).

4. If $j=k+1$, then stop; otherwise set $j=j+1$ and go to step 2 .

${ }^{17}$ This simple example is in fact one of the two largest $k=5$ upper-echelon classes in which there are eight $\mu^{\prime}$ s equivalent to the upper-echelon form. 


\section{Example 2.}

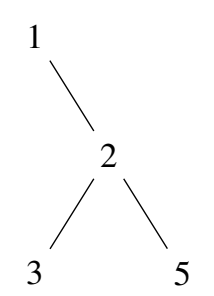

Let us work with the following example ${ }^{18}$

$$
\begin{array}{c|ccccc}
j & 2 & 3 & 4 & 5 & 6 \\
\hline \mu_{\text {out }} & 1 & 1 & 1 & 2 & 3
\end{array}
$$

We start with $j=2$, and note that $\mu_{\text {out }}(2)=1$ so need to find minimal $a>2$, $b>2$ such that $\mu(a)=1$ and $\mu(b)=2$. In this case, it is $a=3$ and $b=5$, so we put those as left and right children of 2 , respectively, in the tree (shown at left)

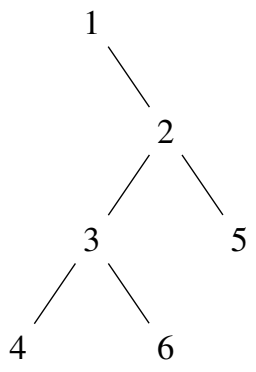

Now we move to $j=3$. Since $\mu_{\text {out }}(3)=1$, we find minimal $a$ and $b$ so that $a>3, b>3, \mu(a)=1$ and $\mu(b)=3$. We find that $a=4$ and $b=6$, so we put these as the left and right children of 3 , respectively, in the tree shown at left. Since all indices appear in the tree, it is complete.

Definition 4.1. A binary tree is called an admissible tree if every child node's label is strictly larger than its parent node's label. ${ }^{19}$ For an admissible tree, we call the graph of the tree without any labels in its nodes the skeleton of the tree.

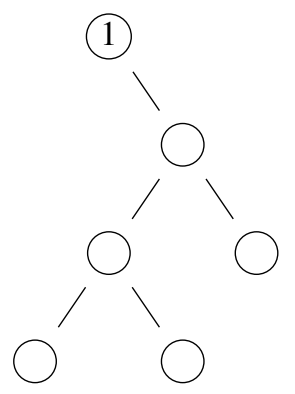

For example, the skeleton of the tree in Example 2 is shown at left.

By the hierarchy structure, Algorithm 2, which produces a tree from a $\mu$, produces only admissible trees. As we have made a distinction between left and right children in the algorithm, the procedure is reversible - given an admissible binary tree, we can uniquely reconstruct the $\mu$ that generated it.

\section{Algorithm 3.}

1. For every right child, $\mu$ maps the child value to the parent value (that is, iff is a right child of $d$, then $\mu(f)=d)$. Start by filling these into the $\mu$ table.

2. Fill in the table using the fact that for every left child, $\mu$ maps the child value to $\mu$ (the parent value).

Example 3. Suppose we are given the following tree:

\footnotetext{
${ }^{19}$ This is certainly a natural requirement coming from the hierarchy.
} 


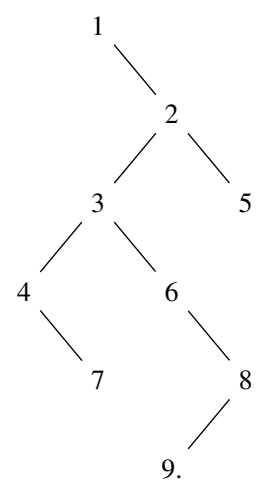

Using the fact that for every right child, $\mu$ maps the child value to the parent value, we fill in the following values in the $\mu$ table:

$$
\begin{array}{r|rrrrrrrr}
j & 2 & 3 & 4 & 5 & 6 & 7 & 8 & 9 \\
\hline \mu & 1 & & & 2 & 3 & 4 & 6 &
\end{array}
$$

Now we use the left-child rule and note that since 3 is a left child of 2 and $\mu(2)=1$, we must have $\mu(3)=1$, and so on, to recover the following table:

$$
\begin{array}{l|llllllll}
j & 2 & 3 & 4 & 5 & 6 & 7 & 8 & 9 \\
\hline \mu & 1 & 1 & 1 & 2 & 3 & 4 & 6 & 6
\end{array}
$$

One can show that in the tree representation of $\mu$, an acceptable move defined in [47] is the operation which switches the labels of two nodes with consecutive labels on an admissible tree, provided that the outcome is still an admissible tree, by writing out the related trees on [47, pp. 180-182]. For example, interchanging the labelling of 5 and 6 in the tree in Example 2 is an acceptable move. That is, acceptable moves in [47] preserve the tree structures but permute the labelling under the admissibility requirement. Two collapsing maps $\mu$ and $\mu^{\prime}$ are KM-relatable if and only if the trees corresponding to $\mu$ and $\mu^{\prime}$ have the same skeleton.

Given $k$, we would like to have the number of different binary tree structures of $k$ nodes. This number is exactly defined as the Catalan number and is controlled by $4^{k}$. Hence, we have just provided a proof of the original KM board game, neglecting the trees showing the effects of acceptable moves on a tree.

But now let us get to the main 'elaborate' part, namely, how to compute $D_{m}$ for a given upperechelon class. To this end, we need to define what is an upper-echelon form. Though the requirement $\mu(j) \leq \mu(j+1)$ for $2 \leq j \leq k$ is good enough, we give an algorithm which produces the upper-echelon tree given the tree structure, as the tree representation of an upper-echelon form is in fact labelled in sequential order (see, for example, the tree in Example 2).

\section{Algorithm 4.}

1. Given a tree structure with $k$ nodes, label the top node with 2 and set a counter $j=2$.

2. If $j=k+1$, then stop; otherwise, continue.

3. If the node labelled $j$ has a left child, then label that left child node with $j+1$, set a counter $j=j+1$ and go to step 2. If not, continue. ${ }^{20}$

4. In the already-labelled nodes which have an empty right child, search for the node with the smallest label. If such a node can be found, label that node's empty right child as $j+1$, set a counter $j=j+1$ and go to step 2. If none of the labelled nodes has an empty right child, then stop.

Definition 4.2. We say $\mu$ is in upper-echelon form if $\mu(j) \leq \mu(j+1)$ for $2 \leq j \leq k$ or if its corresponding tree given by Algorithm 2 agrees with the tree with the same skeleton given by Algorithm 4 .

We define a map $T_{D}$ which maps an upper-echelon tree to a time integration domain (a set of inequality relations) by

$$
T_{D}(\alpha)=\left\{t_{j} \geq t_{k}: j, k \text { are labels on nodes of } \alpha \text { such that the } k \text { node is a child of the } j \text { node }\right\},
$$

where $\alpha$ is an upper-echelon tree. We then have the integration domain as follows:

\footnotetext{
${ }^{20}$ The difference between the definitions of left and right children in Algorithm 2 makes all the enumeration algorithms in this paper address left branches first. See also Section 4.3 for the enumeration of the tamed form.
} 
Proposition 4.3. Given a $\mu_{m}$ in upper-echelon form, we have

$$
\sum_{\mu \sim \mu_{m}} \int_{t_{1} \geq t_{2} \geq t_{3} \geq \cdots \geq t_{k+1}} J_{\mu}^{(k+1)}\left(f^{(k+1)}\right)\left(t_{1}, \underline{t}_{k+1}\right) d \underline{t}_{-k+1}=\int_{T_{D}\left(\mu_{m}\right)} J_{\mu_{m}}^{(k+1)}\left(f^{(k+1)}\right)\left(t_{1}, \underline{t}_{k+1}\right) d \underline{t}_{k+1} .
$$

Here, $\mu \sim \mu_{m}$ means that $\mu$ is equivalent to $\mu_{m}$ under acceptable moves (the trees representing $\mu$ and $\mu_{m}$ have the same structure) and $T_{D}\left(\mu_{m}\right)$ is the domain defined in equation (4.3).

Proof. We prove by an example, as the notation is already heavy. For the general case, one merely needs to rewrite $\Sigma_{1}$ and $\Sigma_{2}$, to be defined in this proof. The key is the admissible condition or the simple requirement that the child must carry a larger label than the parent.

Recall the upper-echelon tree in Example 2 and denote it with $\alpha$. Here are all the admissible trees equivalent to $\alpha$ :

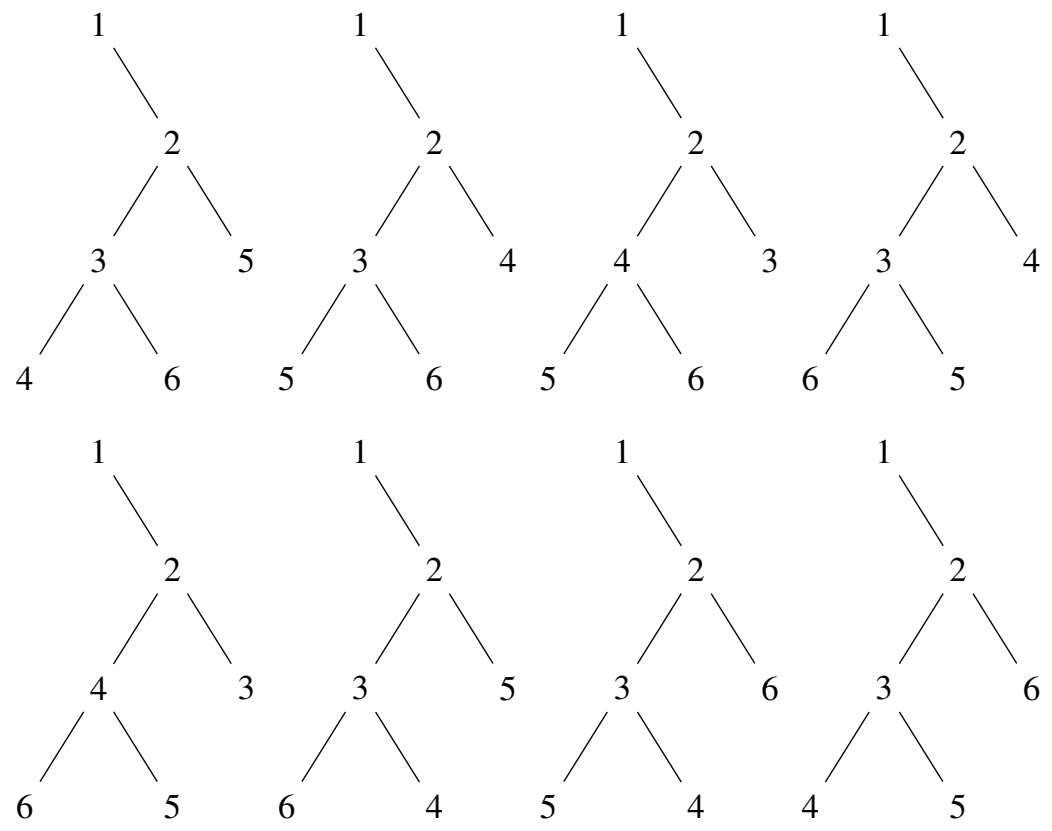

We first read by definition that

$$
T_{D}(\alpha)=\left\{t_{1} \geq t_{2}, t_{2} \geq t_{3}, t_{3} \geq t_{4}, t_{3} \geq t_{6}, t_{2} \geq t_{5}\right\}
$$

Let $\sigma$ denote some composition of acceptable moves. We then notice the equivalence of the two sets

$$
\begin{aligned}
& \Sigma_{1}=\left\{\sigma: \sigma^{-1}(1)<\sigma^{-1}(2)<\sigma^{-1}(3)<\sigma^{-1}(4), \sigma^{-1}(2)<\sigma^{-1}(5), \sigma^{-1}(3)<\sigma^{-1}(6)\right\}, \\
& \Sigma_{2}=\{\sigma: \sigma \text { takes input tree to } \alpha \text { where the input tree is admissible }\},
\end{aligned}
$$

both generated by the requirement that the child must carry a larger label than the parent. That is, both $\Sigma_{1}$ and $\Sigma_{2}$ classify the whole upper-echelon class represented by $\alpha$.

Hence,

$$
\bigcup_{\sigma \in \Sigma_{1}}\left\{t_{1} \geq t_{\sigma(2)} \geq t_{\sigma(3)} \cdots \geq t_{\sigma(6)}\right\}=\left\{t_{1} \geq t_{2} \geq t_{3} \geq t_{4}, t_{2} \geq t_{5}, t_{3} \geq t_{6}\right\}=T_{D}(\alpha),
$$

and we are done. 


\subsection{Signed KM acceptable moves}

While Proposition 4.3 shows that summing over an entire KM upper-echelon class yields a time integration domain with clean structure, it is not sufficient for our purposes. We prove an extended KM board game in Sections 4.2-4.5. Recall the key observation of the KM board game: Many summands in $J^{(k+1)}\left(f^{(k+1)}\right)$ actually have the same integrand if one switches the variable labellings, and thus one can take the acceptable moves to combine them. In fact, one can combine them even more after the acceptable moves to get a larger integration domain $D_{m} \cdot{ }^{21}$ Instead of aiming to reduce the number of summands in $J^{(k+1)}\left(f^{(k+1)}\right)$ even more, our goal this time is to enlarge the integration domain when estimating $J_{\mu, \mathrm{sgn}}^{(k+1)}\left(f^{(k+1)}\right)\left(t_{1}, \underline{t}_{k+1}\right)$, so that $U-V$ techniques can actually apply. Depending on the sign combination in $J_{\mu_{m}, \operatorname{sgn}}^{(k+1)}\left(f^{(k+1)}\right)\left(t_{1}, t_{k+1}\right)$, one could run into the problem of needing to estimate the $x$ part and the $x^{\prime}$ part using the same time integral. This problem is another obstacle stopping $U-V$ space techniques from being used in the analysis of $G P$ hierarchies, separate from the other obstacle that $D_{m}$ was previously unknown.

From here on out, we denote the already unioned or combined integrals in one echelon class as a upper-echelon class integral, and we use Proposition 4.3 for its integration limits. We also put a + or sign at the corresponding node of a tree, as we are dealing with $J_{\mu \text {,sgn }}^{(k+1)}\left(f^{(k+1)}\right)\left(t_{1}, \underline{t}_{k+1}\right)$, in which there are $B^{+}$and $B^{-}$at each coupling. We start with the following example:

Example 4. Let us consider the two upper-echelon trees:
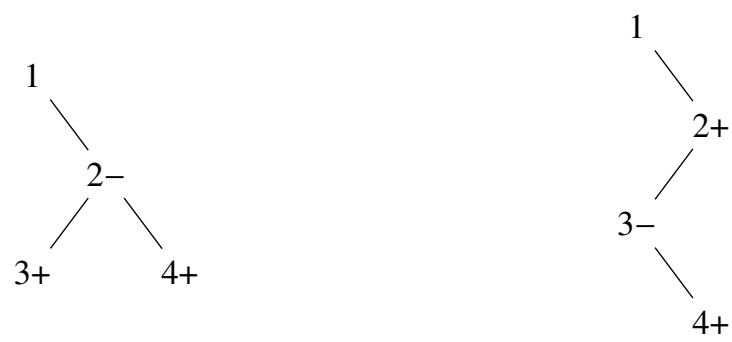

They have the upper-echelon class integrals

$$
\begin{aligned}
& I_{1}=\int_{D_{1}} U^{(1)}\left(t_{1}-t_{2}\right) B_{1,2}^{-} U^{(2)}\left(t_{2}-t_{3}\right) B_{1,3}^{+} U^{(3)}\left(t_{3}-t_{4}\right) B_{2,4}^{+}\left(f^{(4)}\right)\left(t_{1}, \underline{t}_{4}\right) d \underline{t}_{4}, \\
& I_{2}=\int_{D_{2}} U^{(1)}\left(t_{1}-t_{2}\right) B_{1,2}^{+} U^{(2)}\left(t_{2}-t_{3}\right) B_{1,3}^{-} U^{(3)}\left(t_{3}-t_{4}\right) B_{3,4}^{+}\left(f^{(4)}\right)\left(t_{1}, \underline{t}_{4}\right) d \underline{t}_{4},
\end{aligned}
$$

where $D_{1}=\left\{t_{2} \leq t_{1}, t_{3} \leq t_{2}, t_{4} \leq t_{2}\right\}$ and $D_{2}=\left\{t_{2} \leq t_{1}, t_{3} \leq t_{2}, t_{4} \leq t_{3}\right\}$ following from our discussions in Section 4.1.

$I_{1}$ and $I_{2}$ actually have the same integrand if one does a $t_{2} \leftrightarrow t_{3}$ swap in $I_{1}$, despite the fact that the trees corresponding to $I_{1}$ and $I_{2}$ have different skeletons. In fact, shortening $e^{i\left(t_{i}-t_{j}\right) \Delta}$ as $U_{i, j}$, we have

$$
\begin{aligned}
I_{1} & =\int_{D_{1}} U_{1,3}\left(\left|U_{3,4} \phi\right|^{2} U_{3,4} \phi\right)\left(x_{1}\right) U_{1,2}\left(\overline{U_{2,4} \phi U_{2,4} \phi} U_{2,4}\left(|\phi|^{2} \phi\right)\right)\left(x_{1}^{\prime}\right) d \underline{t}_{4} \\
& =\int_{D_{1}^{\prime}} U_{1,2}\left(\left|U_{2,4} \phi\right|^{2} U_{2,4} \phi\right)\left(x_{1}\right) U_{1,3}\left(\overline{U_{3,4} \phi U_{3,4} \phi} U_{3,4}\left(|\phi|^{2} \phi\right)\right)\left(x_{1}^{\prime}\right) d \underline{t}_{4} \\
& =\int_{D_{1}^{\prime}} U^{(1)}\left(t_{1}-t_{2}\right) B_{1,2}^{+} U^{(2)}\left(t_{2}-t_{3}\right) B_{1,3}^{-} U^{(3)}\left(t_{3}-t_{4}\right) B_{3,4}^{+}\left(f^{(4)}\right)\left(t_{1}, \underline{t}_{4}\right) d t_{4},
\end{aligned}
$$

${ }^{21}$ We do not know whether one could combine even more than what we are going to do in Sections 4.2-4.5. 
where $D_{1}^{\prime}=\left\{t_{3} \leq t_{1}, t_{2} \leq t_{3}, t_{4} \leq t_{3}\right\}$ and we have put in $f^{(4)}=(|\phi\rangle\langle\phi|)^{\otimes 4}$ for simplicity. ${ }^{22}$ Hence,

$$
\begin{aligned}
I_{1}+I_{2} & =\int_{D} U^{(1)}\left(t_{1}-t_{2}\right) B_{1,2}^{+} U^{(2)}\left(t_{2}-t_{3}\right) B_{1,3}^{-} U^{(3)}\left(t_{2}-t_{3}\right) B_{3,4}^{+}\left(f^{(4)}\right)\left(t_{1}, \underline{t}_{4}\right) d \underline{t}_{4} \\
& =\int_{t_{4}=0}^{t_{1}} \int_{t_{2}=0}^{t_{1}} \int_{t_{3}=t_{4}}^{t_{1}} U^{(1)}\left(t_{1}-t_{2}\right) B_{1,2}^{+} U^{(2)}\left(t_{2}-t_{3}\right) B_{1,3}^{-} U^{(3)}\left(t_{2}-t_{3}\right) B_{3,4}^{+}\left(f^{(4)}\right)\left(t_{1}, \underline{t}_{4}\right) d \underline{t}_{4},
\end{aligned}
$$

where $D=D_{1}^{\prime} \cup D_{2}=\left\{t_{3} \leq t_{1}, t_{2} \leq t_{1}, t_{4} \leq t_{3}\right\}$.

Example 4 is an easy example of what we will call the wild moves, and shows that one could indeed further combine the summands in $J^{(k+1)}\left(f^{(k+1)}\right)$ after the original KM board game has been performed. We will explain why our $U-V$ techniques apply to $I_{1}+I_{2}$ but not $I_{1}$ and $I_{2}$ individually in Section 5.1. Despite the fact that Example 4 uses the already-combined upper-echelon integrals, our extended KM board game actually starts from scratch - that is, it starts from $\gamma^{(1)}\left(t_{1}\right)$ instead of already-combined upper-echelon integrals - as not all upper-echelon integrals act so nicely under the wild moves. However, it is still a multistep process. We will first switch the terms in $\gamma^{(1)}\left(t_{1}\right)$ into their tamed form via signed KM acceptable moves in Sections 4.2 and 4.3, and then categorise the tamed forms into tamed classes via the wild moves in Sections 4.4 and 4.5.

We now explain the program as follows: As before, start by expanding $\gamma^{(1)}\left(t_{1}\right)$ to coupling level $k$, which generates a sum expansion of $k$ ! terms. But now for each of these $k$ ! terms, expand the collapsing operators $B_{\mu(j), j}^{(j)}$ into + and - components, which introduces $2^{k}$ terms. Thus, in all, we have $2^{k} k$ ! terms, each of which has sign-dependent collapsing operators

$$
\gamma^{(1)}=\sum_{\mu, \mathrm{sgn}} I\left(\mu, \mathrm{id}, \mathrm{sgn}, \gamma^{(k+1)}\right)
$$

where id is the identity permutation on $\{2, \ldots, k+1\}$,

$$
I(\mu, \sigma, \mathrm{sgn})=\int_{t_{1} \geq t_{\sigma(2)} \geq \cdots \geq t_{\sigma(k+1)}} J_{\mu, \mathrm{sgn}}^{(k+1)}\left(\gamma^{(k+1)}\right)\left(t_{1}, \underline{t}_{k+1}\right) d \underline{t}_{k+1}
$$

and $J_{\mu, \mathrm{sgn}}^{(k+1)}$ is defined as in equation (3.10). Equation (4.5) is a sum over all admissible $\mu$ - that is, collapsing maps that satisfy $\mu(j)<j-$ of which there are $k$ !. It is also a sum over all sgn maps, of which there are $2^{k}$.

We define a signed version of the $\mathrm{KM}$ acceptable moves, still denoted $\mathrm{KM}(j, j+1)$, which is defined provided $\mu(j) \neq \mu(j+1)$ and $\mu(j+1)<j$. It is defined as the following action on a triple $(\mu, \sigma, \operatorname{sgn})$ :

$$
\left(\mu^{\prime}, \sigma^{\prime}, \operatorname{sgn}^{\prime}\right)=\operatorname{KM}(j, j+1)(\mu, \sigma, \operatorname{sgn}),
$$

where

$$
\begin{aligned}
\mu^{\prime} & =(j, j+1) \circ \mu \circ(j, j+1), \\
\sigma^{\prime} & =(j, j+1) \circ \sigma, \\
\operatorname{sgn}^{\prime} & =\operatorname{sgn} \circ(j, j+1) .
\end{aligned}
$$

Graphically, this means that nodes $j$ and $j+1$ belong to different left branches and correspond to switching nodes $j$ and $j+1$, leaving the signs in place on the tree - in other words, the node previously labelled $j$ is relabelled $j+1$, and the node previously labelled $j+1$ is relabelled $j$, but the signs are left in place.

${ }^{22}$ One could put a general symmetric $f^{(4)}$ here and get the same result. 
A slight modification of the arguments in [47] shows that, analogous to equation (4.1), if $\left(\mu^{\prime}, \sigma^{\prime}, \operatorname{sgn}^{\prime}\right)=\operatorname{KM}(j, j+1)(\mu, \sigma, \operatorname{sgn})$ and $f^{(k+1)}$ is a symmetric density, then

$$
J_{\mu^{\prime}, \mathrm{sgn}^{\prime}}^{(k+1)}\left(f^{(k+1)}\right)\left(t_{1}, \sigma^{\prime-1}\left(\underline{t}_{k+1}\right)\right)=J_{\mu, \mathrm{sgn}}^{(k+1)}\left(f^{(k+1)}\right)\left(t_{1}, \sigma^{-1}\left(\underline{t}_{k+1}\right)\right) \text {. }
$$

It follows from equation (4.6) that

$$
I\left(\mu^{\prime}, \sigma^{\prime}, \operatorname{sgn}^{\prime}, f^{(k+1)}\right)=I\left(\mu, \sigma, \operatorname{sgn}, f^{(k+1)}\right) .
$$

As in the sign-independent case (or more accurately, the combined-sign case), we can combine KM acceptable moves as follows: If $\rho$ is a permutation of $\{2, \ldots, k+1\}$ such that it is possible to write $\rho$ as a composition of transpositions

$$
\rho=\tau_{1} \circ \cdots \circ \tau_{r}
$$

for which each operator $\operatorname{KM}\left(\tau_{j}\right)$ on the right side of

$$
\mathrm{KM}(\rho) \stackrel{\text { def }}{=} \mathrm{KM}\left(\tau_{1}\right) \circ \cdots \circ \mathrm{KM}\left(\tau_{r}\right)
$$

is an acceptable action, then $\operatorname{KM}(\rho)$, defined by this composition, is acceptable as well. In this case, $\left(\mu^{\prime}, \sigma^{\prime}, \operatorname{sgn}^{\prime}\right)=\operatorname{KM}(\rho)(\mu, \sigma, \operatorname{sgn})$, and

$$
\begin{aligned}
\mu^{\prime} & =\rho \circ \mu \circ \rho^{-1}, \\
\sigma^{\prime} & =\rho \circ \sigma, \\
\operatorname{sgn}^{\prime} & =\operatorname{sgn} \circ \rho^{-1} .
\end{aligned}
$$

Of course, equations (4.6) and (4.7) hold as well. If $(\mu, \operatorname{sgn})$ and $\left(\mu^{\prime}, \operatorname{sgn}^{\prime}\right)$ are such that there exists $\rho$ for which $\left(\mu^{\prime}, \sigma^{\prime}, \operatorname{sgn}^{\prime}\right)=\operatorname{KM}(\rho)(\mu, \sigma, \operatorname{sgn})$, then we say that $\left(\mu^{\prime}, \operatorname{sgn}^{\prime}\right)$ and $(\mu, \operatorname{sgn})$ are KM-relatable. This is an equivalence relation that partitions the set of collapsing map/sign map pairs into equivalence classes. In the graphical representation, two such pairs are KM-relatable if and only if they have the same signed skeleton tree.

Whereas we could use the signed KM acceptable moves to convert an arbitrary admissible $\mu$ to an upper-echelon $\mu^{\prime}$, this will no longer suit our purpose. Instead, our program will be to convert each pair $(\mu, \mathrm{sgn})$ to a tamed form, which we define in the next section. The reason for our preference of tamed form over upper-echelon form is that it is invariant under wild moves, to be introduced in Section 4.4.

\subsection{Tamed form}

In this section, we define what it means for a pair $(\mu, \operatorname{sgn})$ and its corresponding tree representation to be tamed, in Definition 4.4. Then through an example, we present an algorithm for producing the tamed enumeration of a signed skeleton. The general algorithm is then stated in Algorithm 5. Notice that it produces a different enumeration from Algorithm 4. Compared with Algorithm 4, the tamed-form enumeration deals not just with left branches first, it also deals with + first. ${ }^{23}$ In Section 4.3.1, we exhibit how to reduce a signed tree with the same skeleton but different enumeration into the tamed form using signed KM acceptable moves.

We will now give a nongraphical set of conditions on $\mu$ and sgn that determine whether or not ( $\mu, \operatorname{sgn})$ is tamed. First, we define the concept of a tier. We say that $j \geq 2$ is of tier $q$ if

$$
\mu^{q}(j)=1 \text { but } \mu^{q-1}(j)>1,
$$

where $\mu^{q}=\mu \circ \cdots \circ \mu$, the composition taken $q$ times. We write $t(j)$ for the tier value of $j$.

\footnotetext{
${ }^{23}$ By symmetry, one could deal with - first here to get a very similar tamed form. But left and right branches are not symmetric, as they are defined differently.
} 
Definition 4.4. A pair $(\mu, \mathrm{sgn})$ is tamed if it meets the following four requirements:

1. If $t(\ell)<t(r)$, then $\ell<r$.

2. If $t(\ell)=t(r), \mu^{2}(\ell)=\mu^{2}(r), \operatorname{sgn}(\mu(\ell))=\operatorname{sgn}(\mu(r))$ and $\mu(\ell)<\mu(r)$, then $\ell<r$.

3. If $t(\ell)=t(r), \mu^{2}(\ell)=\mu^{2}(r), \operatorname{sgn}(\mu(\ell))=+$ and $\operatorname{sgn}(\mu(r))=-$, then $\ell<r$.

4. If $t(\ell)=t(r), \mu^{2}(\ell) \neq \mu^{2}(r)$ and $\mu(\ell)<\mu(r)$, then $\ell<r$.

Note that the statement $\mu^{2}(\ell)=\mu^{2}(r)$ means graphically that the parents of $\ell$ and $r$ belong to the same left branch. Conditions (2), (3) and (4) specify the ordering for $\ell$ and $r$ belonging to the same tier, and the rule depends upon whether or not the parents of $\ell$ and $r$ belong to the same left branch. If they do, rule (3) says that a positive parent dominates over a negative parent, but rule (2) says that if the parents are of the same sign, then the ordering follows the parental ordering. Finally, if the parents do not belong to the same left branch, rule (4) says that the ordering follows the parental ordering regardless of the signs of the parents.

Example 5. The $(\mu, \operatorname{sgn})$ pair with tier properties indicated in the following chart is tamed:

\begin{tabular}{c|ccccccccccccc}
$j$ & 2 & 3 & 4 & 5 & 6 & 7 & 8 & 9 & 10 & 11 & 12 & 13 & 14 \\
\hline$\mu(j)$ & 1 & 1 & 1 & 1 & 5 & 5 & 2 & 2 & 7 & 7 & 9 & 9 & 8 \\
$\operatorname{sgn}(j)$ & - & - & + & + & - & + & - & + & - & + & + & - & + \\
$t(j)$ & 1 & 1 & 1 & 1 & 2 & 2 & 2 & 2 & 3 & 3 & 3 & 3 & 3
\end{tabular}

All four conditions in Definition 4.4 can be checked from the chart. This is in fact the ( $\mu$, sgn) pair that appears in the example that follows.

In the following example, we illustrate an algorithm for determining the unique tamed enumeration of a signed skeleton tree. After the example is completed, we give the general form of the algorithm.

For the example, we start with the following skeleton, with only the signs indicated. (Recall that KM acceptable moves will leave the signs in place in the tree and change just the numbering of the nodes.) Start by considering all nodes mapping to 1 (the universal ancestor) - this is the left branch attached to 1 that is four nodes long in the order - - ++, and we enumerate it in order as $2,3,4,5$.

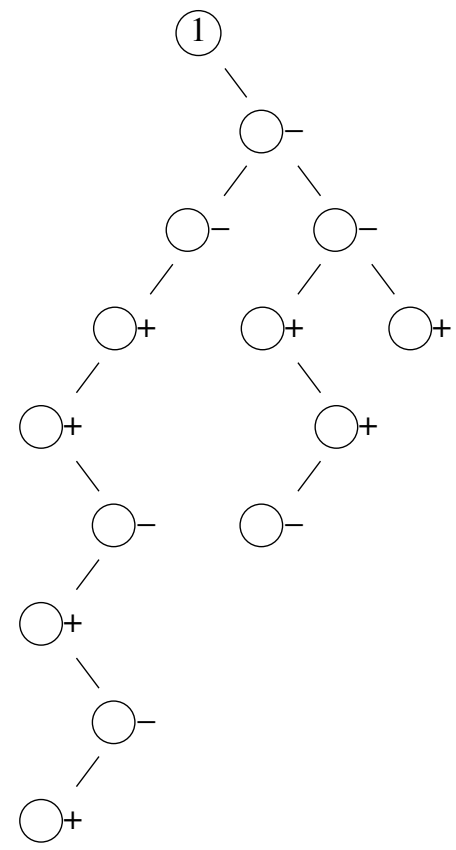




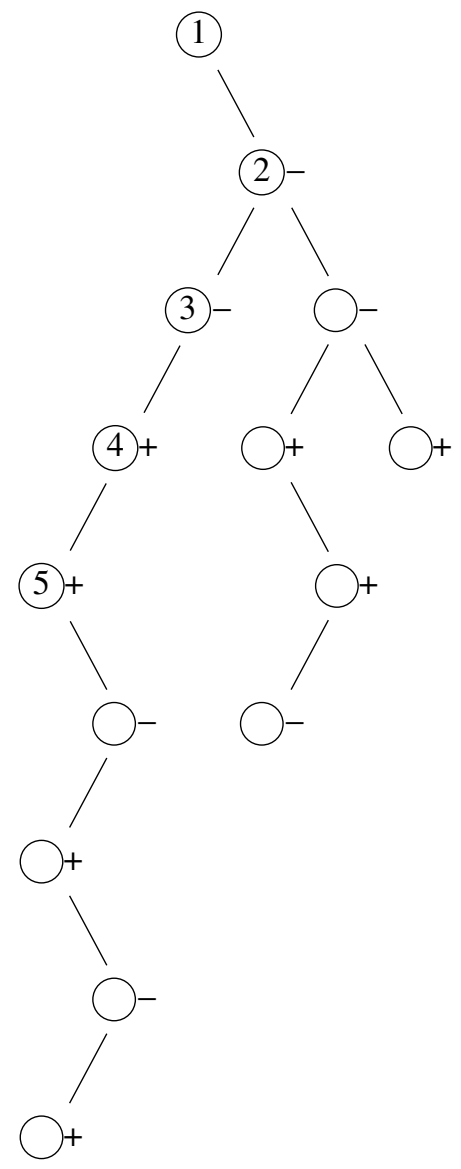

We then put this full left branch in the (empty) queue, listing the + nodes first and then the - nodes:

Queue: 4+, 5+, 2-, 3- .

Then we start working along the queue from left to right. Since 4+ has no right child, we skip it and move to 5+. Since 5+ does have a right child, we label it with the next available number (6) and completely enumerate the entire left branch that starts with this 6 node (that means, in this case, labelling $6-$ and $7+$ as shown on the next graph).

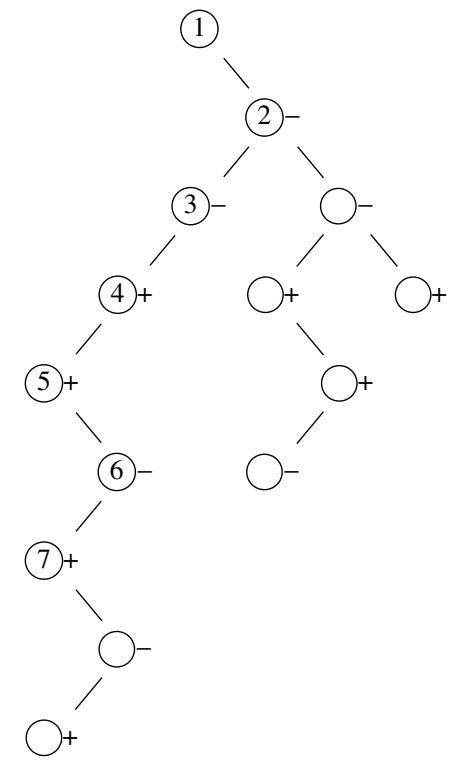


From the queue, we pop 2 and add the $8-, 9+$ left branch - all + nodes first and then all - nodes:

$$
\text { Queue: 3-, 7+, 6-, 9+, 8- . }
$$

Since 3- does not have a right child, we pop it and proceed to $7+$, which does have a right child, which is labelled with 10 , and the left branch starting at 10 is enumerated as 10-, 11+, as shown.

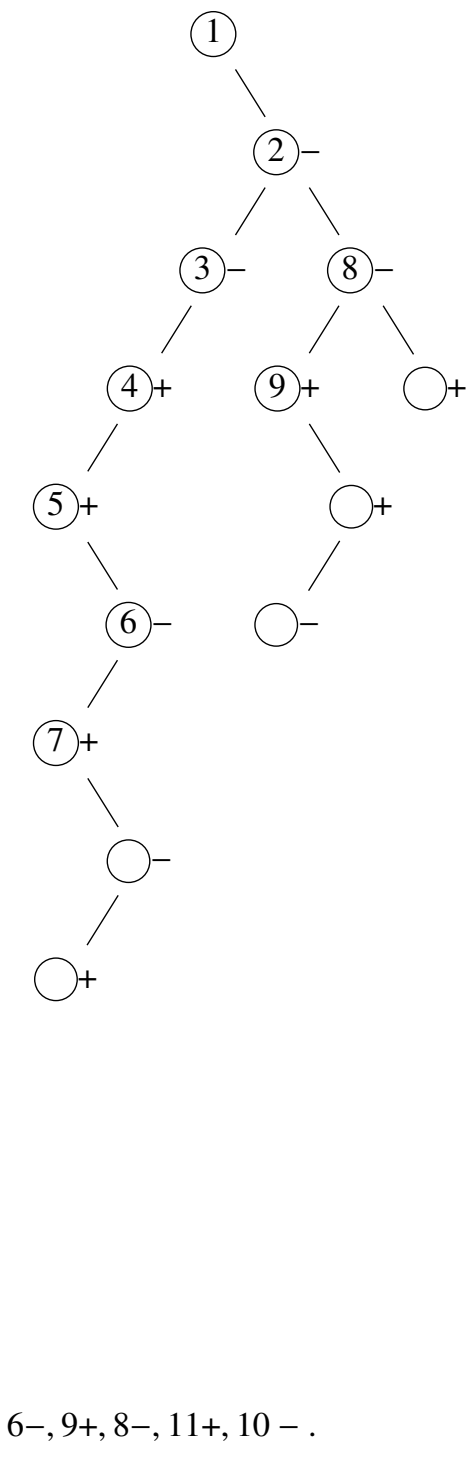

Queue: 6-,9+, 8-, 11+, $10-$.

(5) +

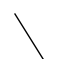

(6)-

1

(7) +

(10)-

(1)
(1)

(2)-

(3)

(4) +

1

- (13)-
(12) +

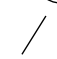

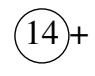

The queue is updated:

By now the procedure is probably clear, so we will jump to the fully enumerated tree. 
Here is the general algorithm. Recall that a queue is a data structure where elements are added on the right and removed (dequeued) on the left.

Algorithm 5. Start with a queue that at first contains only 1 , and start with a next available label $j=2$.

1. Dequeue the leftmost entry $\ell$ of the queue. (If the queue is empty, stop.) On the tree, pass to the right child of $\ell$, and enumerate its left branch starting with the next available label $j, j+1, \ldots, j+q$. If there is no right child of $\ell$, return to the beginning of step 1 .

2. Take the left branch enumerated in step 1 and first list all + nodes in the order $j, \ldots, j+q$ and add them to the right side of the queue. Then list all - nodes in the order $j, \ldots, j+q$ and add them to the right side of the queue

3. Set the next available label to be $j+q+1$, and return to step 1 .

\subsubsection{Reducing to tamed forms via the signed KM board game}

We will now explain how to execute a sequence of signed KM acceptable moves that will bring the example tree from the previous section, with some other enumeration, into the tamed form. This tree corresponds to the following $\mu$ and sgn functions:

\begin{tabular}{c|ccccccccccccc}
$j$ & 2 & 3 & 4 & 5 & 6 & 7 & 8 & 9 & 10 & 11 & 12 & 13 & 14 \\
\hline $\operatorname{sgn}(j)$ & - & - & + & - & + & + & + & - & - & + & + & - & + \\
$\mu(j)$ & 1 & 1 & 1 & 2 & 2 & 1 & 6 & 7 & 6 & 7 & 5 & 11 & 11
\end{tabular}

(1)

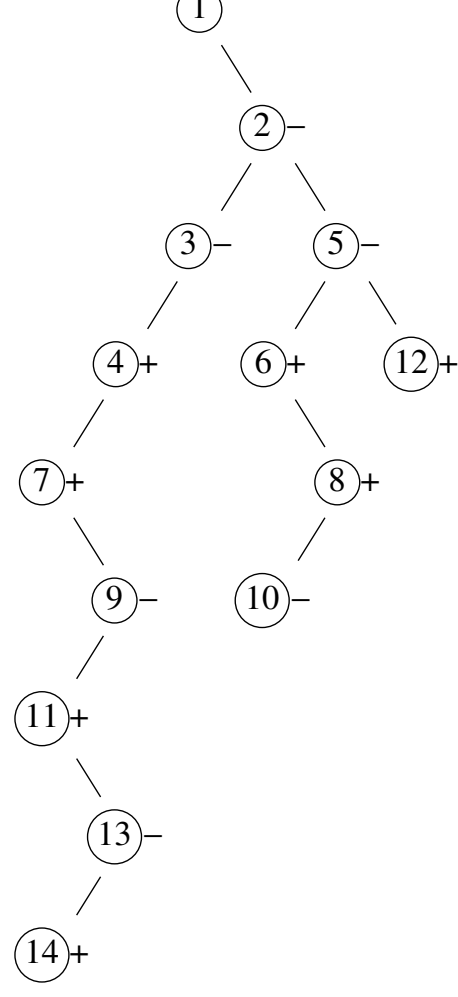

We are going to start with the enumeration at left, which is not tamed, and explain how to execute KM acceptable moves in order to convert this tree into tamed form. Of course, this is quite similar to what Klainerman and Machedon described, with just a modification to prioritise plusses over minuses. We will keep a queue that right now includes only the node 1 :

Queue: 1.

Following the queue, we move all nodes (all $j$ ) for which $\mu(j)=1$ all the way to left using KM moves. Since $\mu(7)=1$, although $\mu(5)=2$ and $\mu(6)=2$, we apply the KM moves $\operatorname{KM}(6,7)$ and then $\operatorname{KM}(5,6)$.

The $\operatorname{KM}(6,7)$ move is

$$
\begin{aligned}
\mu & \mapsto(6,7) \circ \mu \circ(6,7), \\
\operatorname{sgn} & \mapsto \operatorname{sgn} \circ(6,7) .
\end{aligned}
$$

The $\operatorname{KM}(5,6)$ move is

$$
\begin{aligned}
\mu & \mapsto(5,6) \circ \mu \circ(5,6), \\
\operatorname{sgn} & \mapsto \operatorname{sgn} \circ(5,6),
\end{aligned}
$$


and together these result in the following:

\begin{tabular}{c|ccccccccccccc}
$j$ & 2 & 3 & 4 & 5 & 6 & 7 & 8 & 9 & 10 & 11 & 12 & 13 & 14 \\
\hline $\operatorname{sgn}(j)$ & - & - & + & + & - & + & + & - & - & + & + & - & + \\
$\mu(j)$ & 1 & 1 & 1 & 1 & 2 & 2 & 7 & 5 & 7 & 5 & 6 & 11 & 11
\end{tabular}

(1)

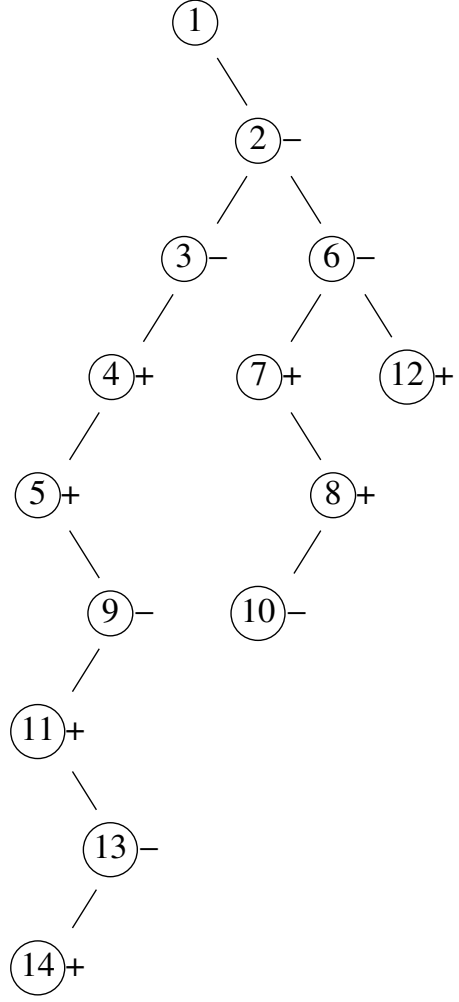

These two moves have been implemented in the revised graph at left.

Inspecting the $\mu$ chart, we see that all output 1 s have been moved to the left, and the complete list of $j$ for which $\mu(j)=1$ is $2-, 3-, 4+, 5+$. We add these numbers to our queue, but first add all plusses and then all minuses:

Queue: 1, 4, 5, 2, 3 .

Since we have completed 1 on the queue, we next move to 4 , but there are no $j$ for which $\mu(j)=4$, so we proceed to 5 . As we can see from the $\mu$ table or from the tree, $\mu(9)=5$ and $\mu(11)=5$, so we execute KM moves to bring these all the way to the left (but to the right of the 1s):

The next step is therefore to implement moves $\operatorname{KM}(8,9), \operatorname{KM}(7,8)$ and $\operatorname{KM}(6,7)$, which brings the $\mu$ table to the following:

\begin{tabular}{c|ccccccccccccc}
$j$ & 2 & 3 & 4 & 5 & 6 & 7 & 8 & 9 & 10 & 11 & 12 & 13 & 14 \\
\hline $\operatorname{sgn}(j)$ & - & - & + & + & - & - & + & + & - & + & + & - & + \\
$\mu(j)$ & 1 & 1 & 1 & 1 & 5 & 2 & 2 & 8 & 8 & 5 & 7 & 11 & 11
\end{tabular}

This is followed by the moves $\operatorname{KM}(11,10), \operatorname{KM}(10,9), \operatorname{KM}(9,8)$ and $\operatorname{KM}(8,7)$, which bring the $\mu$ table to the following:

\begin{tabular}{c|ccccccccccccc}
$j$ & 2 & 3 & 4 & 5 & 6 & 7 & 8 & 9 & 10 & 11 & 12 & 13 & 14 \\
\hline $\operatorname{sgn}(j)$ & - & - & + & + & - & + & - & + & + & - & + & - & + \\
$\mu(j)$ & 1 & 1 & 1 & 1 & 5 & 5 & 2 & 2 & 9 & 9 & 8 & 7 & 7
\end{tabular}




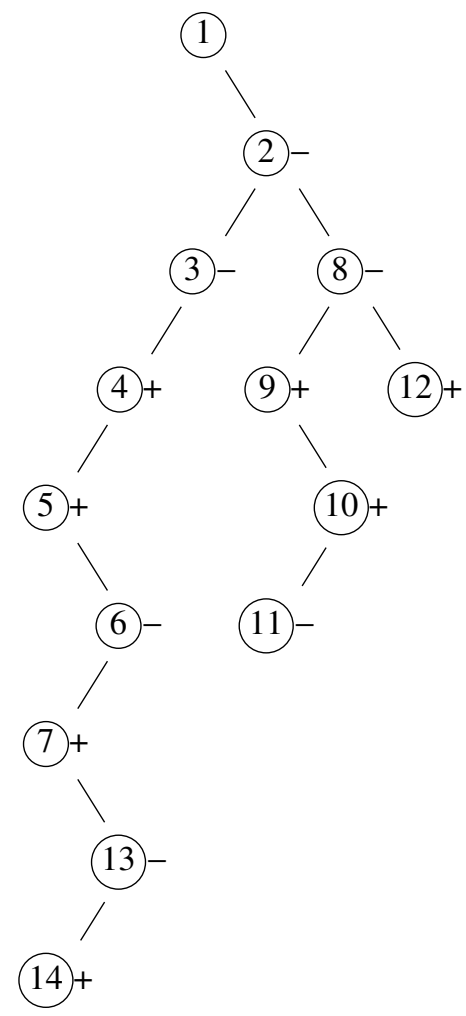

At this point, the tree takes the form as pictured to the left. All 5s have been moved to their proper position in the $\mu$ table. The complete list of $j$ for which $\mu(j)=5$ is 6-,7-, so we add these numbers to the queue, adding the plusses first and then the minuses:

Queue: 1, 4, 5, 2, 3, 7, 6 .

Since we have addressed 5 in the queue, we move to the next item, which is 2 . This means we have to move all $j$ for which $\mu(j)=2$ all the way to the left (just to the right of 5). Examining the $\mu$ table, we see that these $j$ are already in place, at positions 8-, 9+. So no KM moves are needed, and we add to the queue:

$$
\text { Queue: 1, 4, 5, 2, 3, 7, 6, 9, } 8 \text {. }
$$

Next on the queue is 3, but there are no $j$ for which $\mu(j)=3$, so we proceed to 7 on the queue. From the $\mu$ table or the tree, we see there are two $j$ for which $\mu(j)=7$, namely 13 and 14 . We therefore execute KM moves to bring these to the left in the $\mu$ table, just to the right of 2 .

Specifically, we do $\operatorname{KM}(12,13), \operatorname{KM}(11,12)$ and $\operatorname{KM}(10,11)$, which brings us to the following $\mu$ table:

\begin{tabular}{c|ccccccccccccc}
$j$ & 2 & 3 & 4 & 5 & 6 & 7 & 8 & 9 & 10 & 11 & 12 & 13 & 14 \\
\hline $\operatorname{sgn}(j)$ & - & - & + & + & - & + & - & + & - & + & - & + & + \\
$\mu(j)$ & 1 & 1 & 1 & 1 & 5 & 5 & 2 & 2 & 7 & 9 & 9 & 8 & 7
\end{tabular}

After that, we do $\operatorname{KM}(13,14), \operatorname{KM}(12,13)$ and $\operatorname{KM}(11,12)$, which brings us to the following $\mu$ table:

\begin{tabular}{c|ccccccccccccc}
$j$ & 2 & 3 & 4 & 5 & 6 & 7 & 8 & 9 & 10 & 11 & 12 & 13 & 14 \\
\hline $\operatorname{sgn}(j)$ & - & - & + & + & - & + & - & + & - & + & + & - & + \\
$\mu(j)$ & 1 & 1 & 1 & 1 & 5 & 5 & 2 & 2 & 7 & 7 & 9 & 9 & 8
\end{tabular}

Now that the seven outputs are in place, we take the set of $j$ for which $\mu(j)=7$, which is $10-, 11+$, and put them in the queue with plusses first, followed by minuses:

Queue: $1,4,5,2,3,7,6,9,8,11,10$.

There are no $j$ for which $\mu(j)=6$, so we proceed in the queue to 9. However, the two 9s are already in place, and the next item in the queue is 8 ; the one 8 is already in place. So this completes the example.

We now describe the preceding algorithm in general. 
Algorithm 6. Given ( $\mu$, sgn), start with a queue $Q$ that initially contains 1 and a marker $j$, which is initially set to $j=2$. Repeat the following steps:

1. Dequeue the leftmost entry $\ell$ of the queue. If the queue is empty, then stop. Clear the temporary ordered list $L$.

2. If $\mu(j)=\ell$, add $j$ to the right of $L$, then increment the marker $j$ by 1 (so now $j$ is the old $j+1$ ). If (the new marker) $j$ is out of range, jump to step 4 . If $\mu(j) \neq \ell$, then proceed to step 3; otherwise, repeat step 2.

3. Find the smallest $r \geq j+1$ such that $\mu(r)=\ell$ (if there is no such $r$, jump to step 4). Execute signed $K M$ moves $K M(r-1, r)$, followed by $K M(r-2, r-1), \ldots$, until $K M(j+1, j)$. Now $\mu(j)=\ell$. Return to step 2.

4. Take all elements of the temporary ordered list L, read all + entries in order (from left to right) and add them to the (right end of the) queue $Q$; then read all - entries in order (from left to right) and add them to the (right end of the) queue Q. Return to step 1.

We have the following adaptation of Proposition 4.3, revised to include sign maps and to reference tamed forms in place of upper-echelon forms.

Proposition 4.5. Within a signed KM-relatable equivalence class of collapsing map/sign map pairs $(\mu, \mathrm{sgn})$, there is a unique tamed $\left(\mu_{*}, \operatorname{sgn}_{*}\right)$. Moreover,

$$
\sum_{(\mu, \operatorname{sgn}) \sim\left(\mu_{*}, \operatorname{sgn}_{*}\right)} I\left(\mu, i d, \operatorname{sgn}, \gamma^{(k+1)}\right)=\int_{T_{D}\left(\mu_{*}\right)} J_{\mu_{*}, \operatorname{sgn}_{*}}\left(\gamma^{(k+1)}\right)\left(t_{1}, \underline{t}_{k+1}\right) d \underline{t}_{k+1},
$$

where $T_{D}\left(\mu_{*}\right)$ is defined in equation (4.3).

To proceed with our program, we divide the expansion (4.5) into sums over signed KM-relatable equivalence classes, and apply equation (4.8) for the sum over each equivalence class. Thus we obtain

$$
\gamma^{(1)}\left(t_{1}\right)=\sum_{\left(\mu_{*}, \operatorname{sgn}_{*}\right) \text { tamed }} \int_{T_{D}\left(\mu_{*}\right)} J_{\mu_{*}, \operatorname{sgn}_{*}}\left(\gamma^{(k+1)}\right)\left(t_{1}, \underline{t}_{k+1}\right) d t_{-k+1} .
$$

The next step will be to round up the tamed pairs $\left(\mu_{*}, \operatorname{sgn}_{*}\right)$ via wild moves, as defined and discussed in the next section. This will produce a further reduction of equation (4.9).

\subsection{Wild moves}

Definition 4.6. A wild move $\mathrm{W}(\rho)$ is defined as follows: Suppose $(\mu, \operatorname{sgn})$ is a collapsing operator/sign map pair in tamed form, and $\{\ell, \ldots, r\}$ is a full left branch - that is,

$$
z \stackrel{\text { def }}{=} \mu(\ell)=\mu(\ell+1)=\cdots=\mu(r),
$$

but $\mu(\ell-1) \neq z$ (or is undefined) and $\mu(r+1) \neq z$ (or is undefined).

Let $\rho$ be a permutation of $\{\ell, \ell+1, \ldots, r\}$ that satisfies the following condition: If $\ell \leq q<s \leq r$ and $\operatorname{sgn}(q)=\operatorname{sgn}(s)$, then $q$ appears before $s$ in the list $\left(\rho^{-1}(\ell), \ldots, \rho^{-1}(r)\right)$ - or equivalently, $\rho(q)<\rho(s)$.

Then the wild move $\mathrm{W}(\rho)$ is defined as an action on a triple $(\mu, \sigma, \operatorname{sgn})$, where

$$
\left(\mu^{\prime}, \sigma^{\prime}, \operatorname{sgn}^{\prime}\right)=W(\rho)(\mu, \sigma, \operatorname{sgn}),
$$

provided

$$
\begin{aligned}
\mu^{\prime} & =\rho \circ \mu=\rho \circ \mu \circ \rho^{-1}, \\
\sigma^{\prime} & =\rho \circ \sigma, \\
\operatorname{sgn}^{\prime} & =\operatorname{sgn} \circ \rho^{-1} .
\end{aligned}
$$


We note that $W$ is an action

$$
W\left(\rho_{1}\right) W\left(\rho_{2}\right)=W\left(\rho_{1} \circ \rho_{2}\right) .
$$

It is fairly straightforward to show the following, using the definition of a tamed form. It is important to note that the analogous statement for upper-echelon forms does not hold, which is the reason for introducing the tamed class.

Proposition 4.7. Suppose ( $\mu, \mathrm{sgn})$ is a collapsing operator/sign map pair in tamed form, and $W(\rho)$ is a wild move. Letting $\left(\mu^{\prime}, \mathrm{sgn}^{\prime}\right)$ be the output,

$$
\left(\mu^{\prime}, \sigma^{\prime}, \operatorname{sgn}^{\prime}\right)=W(\rho)(\mu, \sigma, \operatorname{sgn}),
$$

then $\left(\mu^{\prime}, \mathrm{sgn}^{\prime}\right)$ is also tamed.

Thus wild moves preserve the tamed class, and we can say that two tamed forms $(\mu, \operatorname{sgn})$ and $\left(\mu^{\prime}, \operatorname{sgn}^{\prime}\right)$ are wildly relatable if there exists $\rho$ as in Definition 4.6 such that

$$
\left(\mu^{\prime}, \sigma^{\prime}, \operatorname{sgn}^{\prime}\right)=W(\rho)(\mu, \sigma, \operatorname{sgn}) .
$$

This is an equivalence relation, and in the sum (4.9) we can partition the class of tamed pairs ( $\mu$, sgn) into equivalence classes of wildly relatable forms (we pursue this in the next section).

The main result of this section is the following:

Proposition 4.8. Suppose that $\rho$ is as in Definition 4.6 and

$$
\left(\mu^{\prime}, \sigma^{\prime}, \operatorname{sgn}^{\prime}\right)=W(\rho)(\mu, \sigma, \operatorname{sgn}) .
$$

Then for any symmetric density $f^{(k+1)}$,

$$
J_{\mu^{\prime}, \mathrm{sgn}^{\prime}}\left(f^{(k+1)}\right)\left(t_{1}, \sigma^{-1}\left(\underline{t}_{k+1}\right)\right)=J_{\mu, \operatorname{sgn}}\left(f^{(k+1)}\right)\left(t_{1}, \sigma^{-1}\left(\underline{t}_{k+1}\right)\right) .
$$

Consequently, the Duhamel integrals are preserved, after adjusting for the time permutations

$$
\int_{\sigma^{\prime}\left[T_{D}\left(\mu^{\prime}\right)\right]} J_{\mu^{\prime}, \mathrm{sgn}^{\prime}}\left(\gamma^{(k+1)}\right)\left(t_{1}, \underline{t}_{k+1}\right) d \underline{t}_{k+1}=\int_{\sigma\left[T_{D}(\mu)\right]} J_{\mu, \operatorname{sgn}}\left(\gamma^{(k+1)}\right)\left(t_{1}, \underline{t}_{k+1}\right) d \underline{t}_{k+1},
$$

where $\sigma\left[T_{D}(\mu)\right]$ is defined by modifying equation (4.3) so that node labels are pushed forward by $\sigma$ :

$$
\begin{array}{r}
\sigma\left[T_{D}(\mu)\right]=\left\{t_{\sigma(j)} \geq t_{\sigma(k)}: j, k \text { are labels on nodes of } \alpha\right. \text { such } \\
\text { that the } k \text { node is a child of the } j \text { node }\} .
\end{array}
$$

Proof. A permutation $\rho$ of the type described in Definition 4.6 can be written as a composition of permutations

$$
\rho=\tau_{1} \circ \cdots \circ \tau_{s}
$$

with the property that each $\tau=(i, i+1)$ for some $i \in\{\ell, \ldots, \ell+r\}$ and $\operatorname{sgn}(i) \neq \operatorname{sgn}(i+1)$. Thus it suffices to prove

$$
\begin{aligned}
U^{(i-1)}\left(-t_{i}\right) B_{\mu(i), i}^{-} U^{(i)}\left(t_{i}-t_{i+1}\right) B_{\mu(i+1), i+1}^{+} & U^{(i+1)}\left(t_{i+1}\right) \\
& =U^{(i-1)}\left(-t_{i+1}\right) B_{\mu(i), i}^{+} U^{(i)}\left(t_{i+1}-t_{i}\right) B_{\mu(i+1), i+1}^{-} U^{(i+1)}\left(t_{i}\right)
\end{aligned}
$$


when the two sides act on a symmetric density. Recall that $z=\mu(i)=\mu(i+1)$. Without loss, we might as well take $z=1$ and $i=2$ so that this becomes

$$
U^{(1)}\left(-t_{2}\right) B_{1,2}^{-} U^{(2)}\left(t_{2}-t_{3}\right) B_{1,3}^{+} U^{(3)}\left(t_{3}\right)=U^{(1)}\left(-t_{3}\right) B_{1,2}^{+} U^{(2)}\left(t_{3}-t_{2}\right) B_{1,3}^{-} U^{(3)}\left(t_{2}\right) .
$$

To prove equation (4.10), on the left side we proceed as follows: First we plug in

$$
\begin{aligned}
U^{(1)}\left(-t_{2}\right) & =U_{-2}^{1} U_{2}^{1^{\prime}} \\
U^{(2)}\left(t_{2}-t_{3}\right) & =U_{2}^{1} U_{-3}^{1} U_{-2}^{1^{\prime}} U_{3}^{1^{\prime}} U_{2}^{2} U_{-3}^{2} U_{-2}^{2^{\prime}} U_{3}^{2^{\prime}} \\
U^{(3)}\left(t_{3}\right) & =U_{3}^{1} U_{-3}^{1^{\prime}} U_{3}^{2} U_{-3}^{2^{\prime}} U_{3}^{3} U_{-3}^{3^{\prime}},
\end{aligned}
$$

where the subscript indicates the time variable and the superscript indicates the spatial variable. Then we note that for the two collapsing operators on the left side of equation (4.10), the following hold:

$\circ B_{1,2}^{-}$acts only on the $2,2^{\prime}$ and $1^{\prime}$ coordinates, so we can move all $U^{1}$ operators in the middle to the left.

$\circ B_{1,3}^{+}$acts only on the $3,3^{\prime}$ and 1 coordinates, so we can move all $U^{2}, U^{2^{\prime}}$ and $U^{1^{\prime}}$ operators in the middle to the right.

This results in

$$
\text { left side of equation }(4.10)=U_{-3}^{1} U_{2}^{1^{\prime}} B_{1,2}^{-} B_{1,3}^{+} U_{3}^{1} U_{-2}^{1^{\prime}} U_{2}^{2} U_{-2}^{2^{\prime}} U_{3}^{3} U_{-3}^{3} \text {. }
$$

Similarly, on the right side of equation (4.10), plug in

$$
\begin{aligned}
U^{(1)}\left(-t_{3}\right) & =U_{-3}^{1} U_{3}^{1^{\prime}} \\
U^{(2)}\left(t_{3}-t_{2}\right) & =U_{3}^{1} U_{-2}^{1} U_{-3}^{1^{\prime}} U_{2}^{1^{\prime}} U_{3}^{2} U_{-2}^{2} U_{-3}^{2^{\prime}} U_{2}^{2^{\prime}} \\
U^{(3)}\left(t_{2}\right) & =U_{2}^{1} U_{-2}^{1^{\prime}} U_{2}^{2} U_{-2}^{2^{\prime}} U_{2}^{3} U_{-2}^{3^{\prime}} .
\end{aligned}
$$

Then we note that for the two collapsing operators on the right side of equation (4.10), the following are true:

○ $B_{1,2}^{+}$acts only on the $2,2^{\prime}$ and 1 coordinates, so we can move all $U^{1^{\prime}}$ operators in the middle to the left.

$\circ B_{1,3}^{-}$acts only on the $3,3^{\prime}$ and $1^{\prime}$ coordinates, so we can move all $U^{2}, U^{2^{\prime}}$ and $U^{1}$ operators in the middle to the right.

This results in

$$
\text { right side of equation }(4.10)=U_{-3}^{1} U_{2}^{1^{\prime}} B_{1,2}^{+} B_{1,3}^{-} U_{3}^{1} U_{-2}^{1^{\prime}} U_{3}^{2} U_{-3}^{2^{\prime}} U_{2}^{3} U_{-2}^{3^{\prime}} \text {. }
$$

Since equations (4.11) and (4.12) are equal when applied to a symmetric density, this proves equation (4.10). In particular, one just needs that to permute

$$
\left(x_{2}, x_{2}^{\prime}, x_{3}, x_{3}^{\prime}\right) \leftrightarrow\left(x_{3}, x_{3}^{\prime}, x_{2}, x_{2}^{\prime}\right) .
$$

Example 6. The pair $\left(\mu_{1}, \operatorname{sgn}_{1}\right)$ is defined as follows:

\begin{tabular}{c|cccccc} 
& 2 & 3 & 4 & 5 & 6 & 7 \\
\hline$\mu_{1}$ & 1 & 1 & 1 & 2 & 4 & 4 \\
$\operatorname{sgn}_{1}$ & + & + & - & - & + & -
\end{tabular}


There are five nontrivial wild moves for $j=2, \ldots, 6$,

$$
\left(\mu_{j}, \sigma_{j}, \operatorname{sgn}_{j}\right)=W\left(\rho_{j}\right)\left(\mu_{1}, \mathrm{id}, \operatorname{sgn}_{1}\right)
$$

as indicated in the following table:

\begin{tabular}{l|lll|ll||l|lll|ll} 
& 2 & 3 & 4 & 6 & 7 & & 2 & 3 & 4 & 6 & 7 \\
\hline$\rho_{1}$ & 2 & 3 & 4 & 6 & 7 & $\rho_{1}^{-1}$ & 2 & 3 & 4 & 6 & 7 \\
$\rho_{2}$ & 2 & 4 & 3 & 6 & 7 & $\rho_{2}^{-1}$ & 2 & 4 & 3 & 6 & 7 \\
$\rho_{3}$ & 3 & 4 & 2 & 6 & 7 & $\rho_{3}^{-1}$ & 4 & 2 & 3 & 6 & 7 \\
$\rho_{4}$ & 2 & 3 & 4 & 7 & 6 & $\rho_{4}^{-1}$ & 2 & 3 & 4 & 7 & 6 \\
$\rho_{5}$ & 2 & 4 & 3 & 7 & 6 & $\rho_{5}^{-1}$ & 2 & 4 & 3 & 7 & 6 \\
$\rho_{6}$ & 3 & 4 & 2 & 7 & 6 & $\rho_{6}^{-1}$ & 4 & 2 & 3 & 7 & 6
\end{tabular}

Notice that each $\rho_{j}^{-1}$ preserves the order of 2,3, as in Definition 4.6 - meaning that 2 appears before 3 in the list $\left(\rho_{j}^{-1}(2), \rho_{j}^{-1}(3), \rho_{j}^{-1}(4)\right)$; equivalently, $\rho(2)<\rho(3)$. Thus the action of $\rho^{-1}$ on $\{2,3,4\}$ is completely determined by where 4 appears in the list $\left(\rho_{j}^{-1}(2), \rho_{j}^{-1}(3), \rho_{j}^{-1}(4)\right)$.

The corresponding trees and explicit mappings $\left(\mu_{j}, \operatorname{sgn}_{j}\right)$ are indicated in the following. We notice that all $\left(\mu_{j}, \operatorname{sgn}_{j}\right)$ are tamed (in accordance with Proposition 4.7) and that wild moves, unlike KM moves, do change the tree skeleton, but this change is restricted to shuffling nodes along a left branch, subject to the restrictions (indicated in Definition 4.6) that the ordering of the plus nodes and minus nodes remain intact.
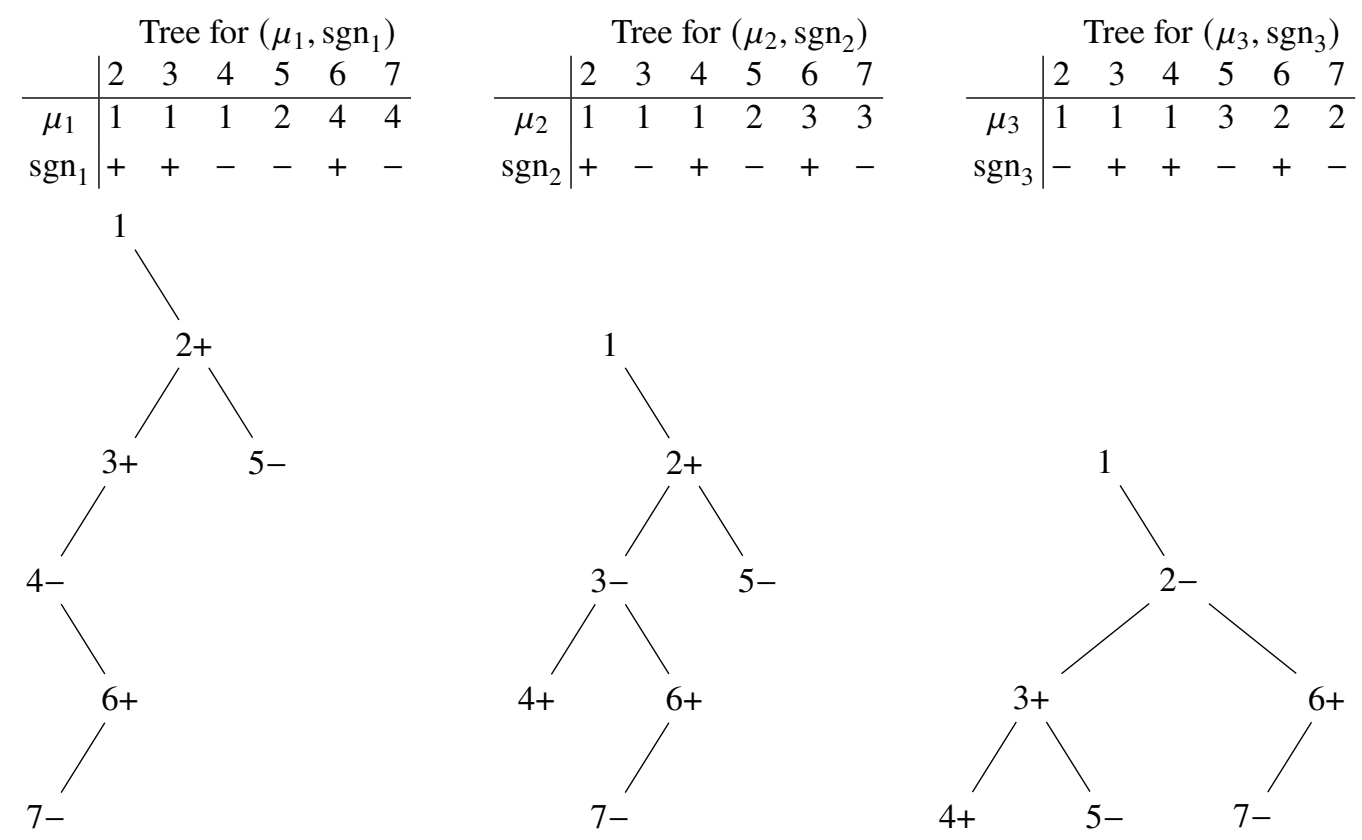

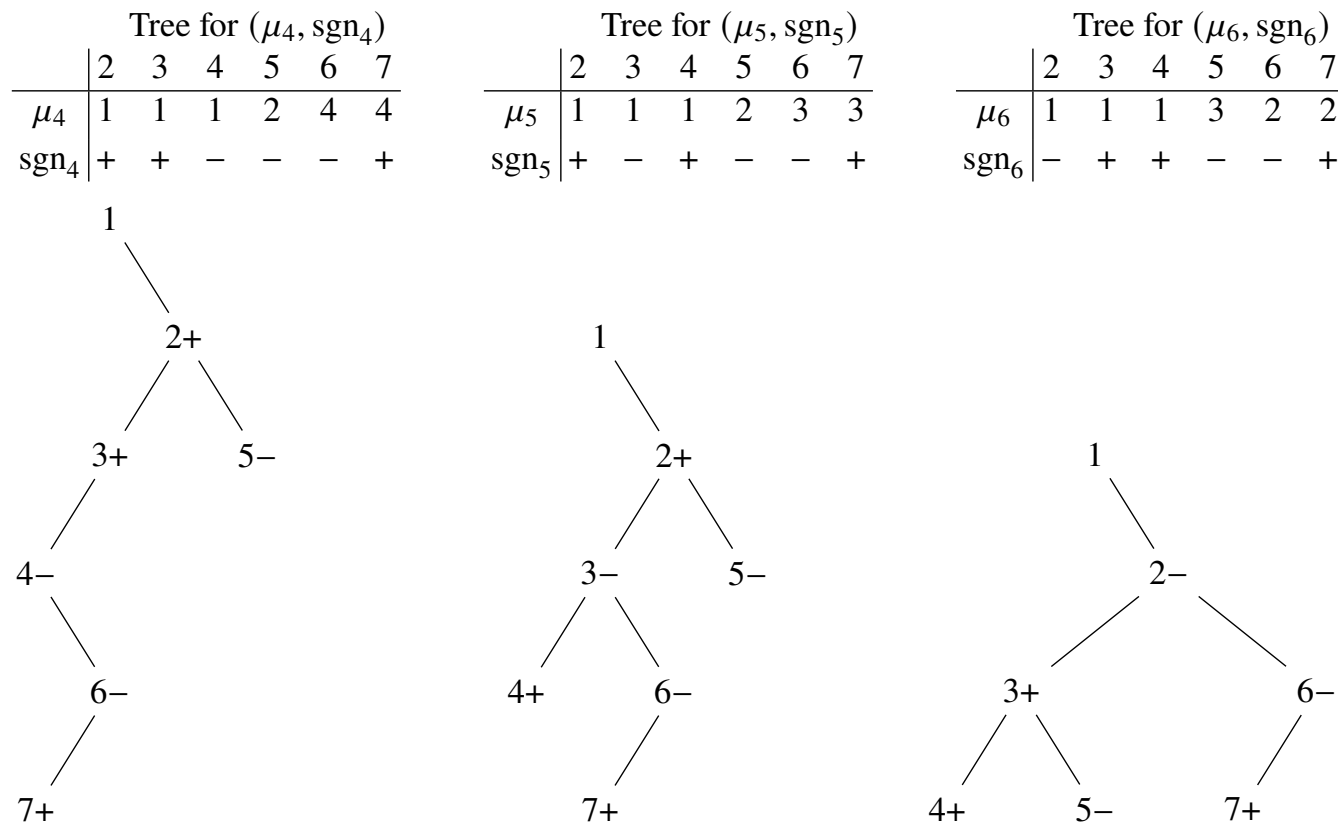

\subsection{Reference forms and tamed integration domains}

Definition 4.9. A tamed pair ( $\hat{\mu}, \mathrm{s} \hat{\mathrm{g} n})$ will be called a reference pair provided that in every left branch, all the + nodes come before all the - nodes.

Definition 4.10. Given a reference pair ( $\hat{\mu}$, sĝn), we will call a permutation $\rho$ of $\{2, \ldots, k+1\}$ allowable if it meets the conditions in Definition 4.6 - that is, it leaves all left branches invariant and moreover, for each left branch $(\ell, \ldots, r)$, all + nodes appear in their original order and all - nodes appear in their original order within the list $\left(\rho^{-1}(\ell), \ldots, \rho^{-1}(r)\right)$.

For example, the tree $\left(\mu_{1}, \operatorname{sgn}_{1}\right)$ in Example 6 is a reference pair. If $(\ell, \ldots, r)$ is a full left branch of $\hat{\mu}$, then the definition of a reference pair means that there is some intermediate position $m$ such that the sgn map looks like the following:

$$
\begin{array}{c|ccccccc}
j & \ell & \cdots & m-1 & m & m+1 & \cdots & r \\
\hline \operatorname{sgn} \mid+ & + & + & - & - & - & -
\end{array}
$$

However, we note that it is possible that they are all plusses $(m=r+1)$ or all minuses $(m=\ell)$. With this notation, we can say that $\rho$ is allowable if $\rho(\ell)<\cdots<\rho(m-1)$ and $\rho(m)<\cdots<\rho(r)-$ or equivalently, if in the list

$$
\left(\rho^{-1}(\ell), \ldots, \rho^{-1}(r)\right)
$$

the values $(\ell, \ldots, m-1)$ appear in that order and the values $(m, \ldots, r)$ appear in that order.

Proposition 4.11. An equivalence class of wildly relatable tamed pairs

$$
Q=\{(\mu, \operatorname{sgn})\}
$$


contains a unique reference pair ( $\hat{\mu}, \mathrm{s} \hat{\mathrm{gn}})$. By the definition of being wildly relatable, for every $(\mu, \mathrm{sgn}) \in$ $Q$ there is a unique permutation $\rho$ of $\{2, \ldots, k+1\}$ such that

$$
(\mu, \operatorname{sgn})=W(\rho)(\hat{\mu}, \mathrm{s} \hat{g} n),
$$

and this $\rho$ is allowable. The collection $P$ of all $\rho$ arising in this way from $Q$ is exactly the set of all allowable $\rho$ with respect to the reference pair ( $\hat{\mu}, \mathrm{s} \hat{\mathrm{g} n})$.

Now, recall equation (4.9):

$$
\gamma^{(1)}\left(t_{1}\right)=\sum_{(\mu, \mathrm{sgn}) \text { tamed }} \int_{T_{D}(\mu)} J_{\mu, \mathrm{sgn}}\left(\gamma^{(k+1)}\right)\left(t_{1}, \underline{t}_{k+1}\right) d \underline{t}_{k+1} .
$$

In this sum, group together equivalence classes $Q$ of wildly relatable $(\mu, \operatorname{sgn})$ :

$$
\gamma^{(1)}\left(t_{1}\right)=\sum_{\text {classes } Q} \sum_{(\mu, \sigma) \in Q} \int_{T_{D}(\mu)} J_{\mu, \operatorname{sgn}}\left(\gamma^{(k+1)}\right)\left(t_{1}, \underline{t}_{k+1}\right) d \underline{t}_{-k+1} .
$$

Each class $Q$ can be represented by a unique reference ( $\hat{\mu}, \mathrm{s} \hat{\mathrm{gn}})$, and as in Proposition 4.11, for each $(\mu, \operatorname{sgn}) \in Q$, there is an allowable $\rho \in P$ (with respect to $(\hat{\mu}, \operatorname{sg} n))$ such that

$$
(\mu, \operatorname{sgn})=W(\rho)(\hat{\mu}, \operatorname{sg} n) .
$$

Since $W$ is an action, we can write

$$
(\hat{\mu}, \mathrm{sg} n)=W\left(\rho^{-1}\right)(\mu, \operatorname{sgn}) .
$$

Into the action $W\left(\rho^{-1}\right)$, let us input the identity time permutation and define $\sigma$ as the output time permutation:

$$
(\hat{\mu}, \sigma, \mathrm{s} \hat{\mathrm{gn}})=W\left(\rho^{-1}\right)(\mu, \mathrm{id}, \mathrm{sgn}),
$$

where, in accordance with Definition 4.6, $\sigma=\rho^{-1}$. Since $\rho$ is allowable, this implies that for each left brach $(\ell, \ldots, r)$ with $m$ as already defined, $\sigma^{-1}(\ell)<\cdots<\sigma^{-1}(m-1)$ and $\sigma^{-1}(m)<\cdots<\sigma^{-1}(r)$. In other words, $(\ell, \ldots, m-1)$ and $(m, \ldots, r)$ appear in order inside the list of values $(\sigma(\ell), \ldots, \sigma(r))$. By Proposition 4.8,

$$
\int_{T_{D}(\mu)} J_{\mu, \operatorname{sgn}}\left(\gamma^{(k+1)}\right)\left(t_{1}, \underline{t}_{k+1}\right) d \underline{t}_{k+1}=\int_{\sigma\left[T_{D}(\hat{\mu})\right]} J_{\hat{\mu}, \text { ŝn }}\left(\gamma^{(k+1)}\right)\left(t_{1}, \underline{t}_{k+1}\right) d \underline{t}_{k+1} .
$$

Now as we sum this over all $(\mu, \operatorname{sgn}) \in Q$, we are summing over all $\rho \in P$ and hence over all $\sigma=\rho^{-1}$ meeting the condition already mentioned. Hence the integration domains on the right side union to a set that we will denote

$$
T_{R}(\hat{\mu}, \mathrm{s} \hat{\mathrm{gn}}) \stackrel{\text { def }}{=} \bigcup_{\rho \in P} \sigma\left(T_{D}(\hat{\mu})\right)
$$

which can be described as follows: For each left branch $(\ell, \ldots, r)$, with

$$
z=\mu(\ell)=\cdots=\mu(r)
$$

and $m$ the division index between plus and minus nodes, $T_{R}(\hat{\mu}$, s $\hat{g} n)$ is described by the inequalities

$$
t_{m-1} \leq \cdots \leq t_{\ell} \leq t_{z} \quad \text { and } \quad t_{r} \leq \cdots \leq t_{m} \leq t_{z}
$$


Plugging into equation (4.13), we obtain the following:

Proposition 4.12. The Duhamel expansion to coupling order $k$ can be grouped into at most $8^{k}$ terms:

$$
\gamma^{(1)}\left(t_{1}\right)=\sum_{\text {reference }(\hat{\mu}, \mathrm{sg} \mathrm{n})} \int_{T_{R}(\hat{\mu}, \mathrm{sgn})} J_{\mu, \mathrm{sgn}}\left(\gamma^{(k+1)}\right)\left(t_{1}, \underline{t}_{k+1}\right) d \underline{t}_{k+1} \text {, }
$$

where each integration domain $T_{R}(\hat{\mu}, \mathrm{s} \hat{\mathrm{g}})$ is as defined in formula (4.14).

A quick example of formulas (4.14) and (4.15) is Example 4, in which the reference tree is the one corresponding to $I_{2}$. Reading from that tree, formula (4.14) becomes the set $D$ and the combined integral is equation (4.4).

Returning to Example 6, $\left(\mu_{1}, \mathrm{sgn}_{1}\right)$ is the reference pair. To combine the Duhamel integrals, we convert all five other tamed forms $\left(\mu_{j}, \operatorname{sgn}_{j}\right)$ to $\left(\mu_{1}, \operatorname{sgn}_{1}\right)$ via wild moves. The resulting combined time integration set will be read off from the $\left(\mu_{1}, \operatorname{sgn}_{1}\right)$ tree as

$$
t_{3} \leq t_{2} \leq t_{1}, \quad t_{4} \leq t_{1}, \quad t_{5} \leq t_{2}, \quad t_{6} \leq t_{4}, \quad t_{7} \leq t_{4} .
$$

Proposition 4.12 and the integration domain (4.14) are compatible with the $U$ - $V$ space techniques we proved in Section 2. This fact may not be so clear at the moment, as they are written with much shorthand. We will prove this fact in Section 5.2.

\section{Uniqueness for the GP hierarchy (1.2) - Actual estimates}

The main goal of this section is to prove Proposition 3.7 on estimating $J_{\mu_{m}, \mathrm{sgn}}^{(k+1)}$. Of course, by $J_{\mu_{m}, \mathrm{sgn}}^{(k+1)}$ we mean the reference form now. We first present an example in Section 5.1 to convey the basic ideas of the proof. Then in Section 5.2 we demonstrate why we need the extended KM board game and prove that Proposition 4.12 and the integration domain (4.14) are compatible with the $U-V$ space techniques. Once that is settled, the main idea idea in Section 5.1 will work for the general case. Thus we estimate the general case in Section 5.3.

The time integration limits in Section 4.5 will be put to use with Lemmas 2.1 and 2.2. With the trivial estimate $\|u\|_{Y^{s}} \lesssim\|u\|_{X^{s}}$, Lemmas 2.1 and 2.2 read as

$$
\begin{gathered}
\left\|\int_{a}^{t} e^{-i\left(t-t^{\prime}\right) \Delta}\left(u_{1} u_{2} u_{3}\right)\left(\bullet, t^{\prime}\right) d t^{\prime}\right\|_{X^{-1}} \leq C\left\|u_{1}\right\|_{X^{-1}}\left(T^{\frac{1}{7}} M_{0}^{\frac{3}{5}}\left\|P_{\leq M_{0}} u_{2}\right\|_{X^{1}}+\left\|P_{>M_{0}} u_{2}\right\|_{X^{1}}\right)\left\|u_{3}\right\|_{X^{1}} \\
\left\|\int_{a}^{t} e^{-i\left(t-t^{\prime}\right) \Delta}\left(u_{1} u_{2} u_{3}\right)\left(\bullet, t^{\prime}\right) d t^{\prime}\right\|_{X^{1}} \leq C\left\|u_{1}\right\|_{X^{1}}\left(T^{\frac{1}{7}} M_{0}^{\frac{3}{5}}\left\|P_{\leq M_{0}} u_{2}\right\|_{X^{1}}+\left\|P_{>M_{0}} u_{2}\right\|_{X^{1}}\right)\left\|u_{3}\right\|_{X^{1}} \\
\left\|\int_{a}^{t} e^{-i\left(t-t^{\prime}\right) \Delta}\left(u_{1} u_{2} u_{3}\right)\left(\bullet, t^{\prime}\right) d t^{\prime}\right\|_{X^{-1}} \leq C\left\|u_{1}\right\|_{X^{-1}}\left\|u_{2}\right\|_{X^{1}}\left\|u_{3}\right\|_{X^{1}} \\
\left\|\int_{a}^{t} e^{-i\left(t-t^{\prime}\right) \Delta}\left(u_{1} u_{2} u_{3}\right)\left(\bullet, t^{\prime}\right) d t^{\prime}\right\|_{X^{1}} \leq C\left\|u_{1}\right\|_{X^{1}}\left\|u_{2}\right\|_{X^{1}}\left\|u_{3}\right\|_{X^{1}}
\end{gathered}
$$

If $u_{j}=e^{i t^{\prime} \Delta} f_{j}$ for some $j$ and some $f_{j}$ independent of $t$ and $t^{\prime}$, we can replace the $X^{s}$ norm of $u_{j}$ in formulas (5.1)-(5.4) with the $H^{s}$ norm of $f_{j}$. We do not use $\lesssim$ in these equations because we are going to use them repeatedly, and the constants are going to accumulate. 


\subsection{An example of how to estimate}

We estimate the integral in Example 4:

$$
I=\int_{t_{4}=0}^{t_{1}} \int_{t_{2}=0}^{t_{1}} \int_{t_{3}=t_{4}}^{t_{1}} U^{(1)}\left(t_{1}-t_{2}\right) B_{1,2}^{+} U^{(2)}\left(t_{2}-t_{3}\right) B_{1,3}^{-} U^{(3)}\left(t_{3}-t_{4}\right) B_{3,4}^{+} \gamma^{(4)} d \underline{t}_{4},
$$

where the integration limits have already been computed in Section 4.2. Its reference tree is exactly the tree corresponding to $I_{2}$ in Example 4.

Plugging in equation (3.6), we find that the integrand is in fact

$$
\begin{aligned}
I=\int_{t_{4}=0}^{t_{1}} d t_{4} \int d \mu_{t_{4}}(\phi) \int_{t_{2}=0}^{t_{1}} d t_{2} \int_{t_{3}=t_{4}}^{t_{1}} U_{1,2}\left(\left|U_{2,4} \phi\right|^{2} U_{2,4} \phi\right)\left(x_{1}\right) \\
\quad \times U_{1,3}\left(\overline{U_{3,4} \phi U_{3,4} \phi} U_{3,4}\left(|\phi|^{2} \phi\right)\right)\left(x_{1}^{\prime}\right) d t_{3} .
\end{aligned}
$$

We denote the cubic term $|\phi|^{2} \phi$ generated in the innermost coupling with $\mathcal{C}_{R}^{(4)}$, where the subscript $R$ stands for 'rough', as it has no propagator inside to smooth things out. That is,

$$
I=\int_{t_{4}=0}^{t_{1}} d t_{4} \int d \mu_{t_{4}}(\phi) \int_{t_{2}=0}^{t_{1}} d t_{2} \int_{t_{3}=t_{4}}^{t_{1}} U_{1,2}\left(\left|U_{2,4} \phi\right|^{2} U_{2,4} \phi\right)\left(x_{1}\right) U_{1,3}\left(\overline{U_{3,4} \phi U_{3,4} \phi} U_{3,4} \mathcal{C}_{R}^{(4)}\right)\left(x_{1}^{\prime}\right) d t_{3}
$$

For expression (3.9) with a general $k$, we will use $\mathcal{C}_{R}^{(k+1)}$ to denote this innermost cubic term. Notice that $\mathcal{C}_{R}^{(k+1)}$ is always independent of time and is hence qualified to be an $f_{j}$ in estimates (5.1)-(5.4).

In the second coupling, if we denote

$$
D_{\phi, R}^{(3)}=U_{-t_{3}}\left(\overline{U_{3,4} \phi U_{3,4} \phi} U_{3,4} \mathcal{C}_{R}^{(4)}\right)\left(x_{1}^{\prime}\right)
$$

we have

$$
\int_{t_{3}=t_{4}}^{t_{1}} U_{1,3}\left(\overline{U_{3,4} \phi U_{3,4} \phi} U_{3,4} \mathcal{C}_{R}^{(4)}\right)\left(x_{1}^{\prime}\right) d t_{3}=\int_{t_{3}=t_{4}}^{t_{1}} U_{1} D_{\phi, R}^{(3)} d t_{3}
$$

In general, let us use $D^{(l+1)}$, which is $D_{\phi, R}^{(3)}$ here, to denote the cubic term together with the $U\left(-t_{l+1}\right)$ during the $l$ th coupling where $l<k$. We add a $\phi$ subscript if the cubic term generated at the $l$ th coupling has contracted a $U \phi$. We add an $R$ subscript if the cubic term generated at the $l$ th coupling has contracted the rough cubic term $\mathcal{C}_{R}^{(k+1)}$ or a $D_{R}^{(j+1)}$ for some $j$. The coupling process makes sure that every time integral corresponds to one and only one cubic term, and thus the notation of $D$ is well defined. We suppress all $t_{k+1}$-dependence, which is the $t_{4}$-dependence here, in all the $D$ markings, as we will not explore any smoothing given by the $d t_{k+1}$ integral. Finally, notice that $D^{(l+1)}$ always carries the $t_{l+1}$ variable and will make a Duhamel term whenever it is hit by a $U\left(t_{j}\right)$, where $j \neq l+1$.

Then, using the same marking strategy at the first coupling, we reach

$$
I=\int_{t_{4}=0}^{t_{1}} d t_{4} \int d \mu_{t_{4}}(\phi)\left(\int_{t_{2}=0}^{t_{1}} U_{1} D_{\phi}^{(2)}\left(x_{1}\right) d t_{2}\right)\left(\int_{t_{3}=t_{4}}^{t_{1}} U_{1} D_{\phi, R}^{(3)}\left(x_{1}^{\prime}\right) d t_{3}\right) .
$$

We can now start estimating. Taking the norm inside,

$$
\begin{aligned}
& \left\|\left\langle\nabla_{x_{1}}\right\rangle^{-1}\left\langle\nabla_{x_{1}^{\prime}}\right\rangle^{-1} I\right\|_{L_{t_{1}}^{\infty} L_{x, x^{\prime}}^{2}} \\
\leq & \int_{0}^{T} \int d t_{4} d\left|\mu_{t_{4}}\right|(\phi)\left\|\left(\left\langle\nabla_{x_{1}}\right\rangle^{-1} \int_{t_{2}=0}^{t_{1}} U_{1} D_{\phi}^{(2)}\left(x_{1}\right) d t_{2}\right)\left(\left\langle\nabla_{x_{1}^{\prime}}\right\rangle^{-1} \int_{t_{3}=t_{4}}^{t_{1}} U_{1} D_{\phi, R}^{(3)}\left(x_{1}^{\prime}\right) d t_{3}\right)\right\|_{L_{t_{1}}^{\infty} L_{x, x^{\prime}}^{2}},
\end{aligned}
$$


the $L_{t_{1}}^{\infty} L_{x, x^{\prime}}^{2}$ norm 'factors' in the sense that

$$
\begin{aligned}
\left\|\left\langle\nabla_{x_{1}}\right\rangle^{-1}\left\langle\nabla_{x_{1}^{\prime}}\right\rangle^{-1} I\right\|_{L_{t_{1}}^{\infty} L_{x, x^{\prime}}^{2}} & \\
& \leq \int_{0}^{T} \int\left\|\int_{t_{2}=0}^{t_{1}} U_{1} D_{\phi}^{(2)}\left(x_{1}\right) d t_{2}\right\|_{L_{t_{1}}^{\infty} H_{x}^{-1}}\left\|\int_{t_{3}=t_{4}}^{t_{1}} U_{1} D_{\phi, R}^{(3)}\left(x_{1}^{\prime}\right) d t_{3}\right\|_{L_{t_{1}}^{\infty} H_{x^{\prime}}^{-1}} d t_{4} d\left|\mu_{t_{4}}\right|(\phi) .
\end{aligned}
$$

The term $D_{\phi}^{(2)}$ carries no $R$ subscript, so we can bump it to $H^{1}$ and then use the embedding (2.1), which gives

$$
\begin{array}{rl}
\|\left\langle\nabla_{x_{1}}\right\rangle^{-1}\left\langle\nabla_{x_{1}^{\prime}}\right\rangle^{-1} & I \|_{L_{t_{1}}^{\infty} L_{x, x^{\prime}}^{2}} \\
& \leq \int_{0}^{T} \int\left\|\int_{t_{3}=t_{4}}^{t_{1}} U_{1} D_{\phi, R}^{(3)}\left(x_{1}^{\prime}\right) d t_{3}\right\|_{X^{-1}}\left\|\int_{t_{2}=0}^{t_{1}} U_{1} D_{\phi}^{(2)}\left(x_{1}\right) d t_{2}\right\|_{X^{1}} d t_{4} d\left|\mu_{t_{4}}\right|(\phi) .
\end{array}
$$

Applying formula (5.2) to the first coupling and replacing all $\|U \phi\|_{X^{s}}$ by $\|\phi\|_{H^{s}}$, we have

$$
\begin{aligned}
& \left\|\left\langle\nabla_{x_{1}}\right\rangle^{-1}\left\langle\nabla_{x_{1}^{\prime}}\right\rangle^{-1} I\right\|_{L_{t_{1}}^{\infty} L_{x, x^{\prime}}^{2}} \\
\leq & C \int_{0}^{T} \int\left\|\int_{t_{3}=t_{4}}^{t_{1}} U_{1} D_{\phi, R}^{(3)}\left(x_{1}^{\prime}\right) d t_{3}\right\|_{X^{-1}}\|\phi\|_{H^{1}}^{2}\left(T^{\frac{1}{7}} M_{0}^{\frac{3}{5}}\left\|P_{\leq M_{0}} \phi\right\|_{H^{1}}+\left\|P_{>M_{0}} \phi\right\|_{H^{1}}\right)^{1} d t_{4} d\left|\mu_{t_{4}}\right|(\phi) .
\end{aligned}
$$

Using formula (5.1) with the second coupling and replacing all $\|U \phi\|_{X^{s}}$ by $\|\phi\|_{H^{s}}$, we have

$$
\begin{aligned}
\|\left\langle\nabla_{x_{1}}\right\rangle^{-1} & \left\langle\nabla_{x_{1}^{\prime}}\right\rangle^{-1} I \|_{L_{t_{1}}^{\infty} L_{x, x^{\prime}}^{2}} \\
& \leq C^{2} \int_{0}^{T} \int\|\phi\|_{H^{1}}^{3}\left(T^{\frac{1}{7}} M_{0}^{\frac{3}{5}}\left\|P_{\leq M_{0}} \phi\right\|_{H^{1}}+\left\|P_{>M_{0}} \phi\right\|_{H^{1}}\right)^{2}\left\|\mathcal{C}_{R}^{(4)}\right\|_{H^{-1}} d t_{4} d\left|\mu_{t_{4}}\right|(\phi) \\
& =C^{2} \int_{0}^{T} \int\|\phi\|_{H^{1}}^{3}\left(T^{\frac{1}{7}} M_{0}^{\frac{3}{5}}\left\|P_{\leq M_{0}} \phi\right\|_{H^{1}}+\left\|P_{>M_{0}} \phi\right\|_{H^{1}}\right)^{2}\left\||\phi|^{2} \phi\right\|_{H^{-1}} d t_{4} d\left|\mu_{t_{4}}\right|(\phi) .
\end{aligned}
$$

Using the 4D Sobolev

$$
\left\||\phi|^{2} \phi\right\|_{H^{-1}} \leq C\|\phi\|_{H^{1}}^{3}
$$

on the rough coupling, we get to

$$
\left\|\left\langle\nabla_{x_{1}}\right\rangle^{-1}\left\langle\nabla_{x_{1}^{\prime}}\right\rangle^{-1} I\right\|_{L_{t_{1}}^{\infty} L_{x, x^{\prime}}^{2}} \leq C^{3} \int_{0}^{T} d t_{4} \int d\left|\mu_{t_{4}}\right|(\phi)\|\phi\|_{H^{1}}^{6}\left(T^{\frac{1}{7}} M_{0}^{\frac{3}{5}}\left\|P_{\leq M_{0}} \phi\right\|_{H^{1}}+\left\|P_{>M_{0}} \phi\right\|_{H^{1}}\right)^{2} .
$$

Plugging in the support property of the measure (see equation (3.5)) yields

$$
\begin{aligned}
\left\|\left\langle\nabla_{x_{1}}\right\rangle^{-1}\left\langle\nabla_{x_{1}^{\prime}}\right\rangle^{-1} I\right\|_{L_{t_{1}}^{\infty} L_{x, x^{\prime}}^{2}} & \leq C^{3} C_{0}^{6}\left(T^{\frac{1}{7}} M_{0}^{\frac{3}{5}} C_{0}+\varepsilon\right)^{2} \int_{0}^{T} d t_{4} \int d\left|\mu_{t_{4}}\right|(\phi) \\
& \leq C^{3} C_{0}^{6}\left(T^{\frac{1}{7}} M_{0}^{\frac{3}{5}} C_{0}+\varepsilon\right)^{2} 2 T
\end{aligned}
$$

and we are done. 


\subsection{The extended KM board game is compatible}

In Section 5.1, the $U-V$ estimates worked perfectly with the integration limits obtained via the extended $\mathrm{KM}$ board game from Section 4. One certainly wonders whether the extended KM board game is necessary, and whether it is compatible with the estimates in the general case.

In the beginning of Section 4.2, we briefly mentioned the problem one would face without the extended KM board game. We can now explain by a concrete example. For comparison, rewrite $I_{1}$ in Example 4 with the notation

$$
\begin{aligned}
I_{1} & =\int_{t_{4}=0}^{t_{1}} \int_{t_{2}=t_{4}}^{t_{1}} \int_{t_{3}=0}^{t_{2}} U^{(1)}\left(t_{1}-t_{2}\right) B_{1,2}^{-} U^{(2)}\left(t_{2}-t_{3}\right) B_{1,3}^{+} U^{(3)}\left(t_{3}-t_{4}\right) B_{3,4}^{-} \gamma^{(4)} d t_{4} \\
& =\int_{t_{4}=0}^{t_{1}} d t_{4} \int d \mu_{t_{4}}(\phi) \int_{t_{2}=t_{4}}^{t_{1}}\left(U_{1} D_{\phi}^{(2)}\left(x_{1}\right)\left[\int_{t_{3}=0}^{t_{2}} U_{1} D_{\phi, R}^{(3)}\left(x_{1}^{\prime}\right) d t_{3}\right]\right) d t_{2} .
\end{aligned}
$$

One sees that the $d t_{3}$ integral is encapsulated inside the $d t_{2}$ integral, or the $x$ and $x^{\prime}$ parts do not factor, even with the carefully worked-out time integration limits in the original KM board game. Hence, one cannot apply $U-V$ estimates. To be very precise for readers who are curious about this, since there are only two integrals that got entangled, $I_{1}$ could in fact be estimated using [50, (4.25), p. 60], based on the idea of integration by parts. However, if one allows the coupling level to be large, it is not difficult to find, at any stage of a long coupling, multiple encapsulations which have more than three factors entangled together and cannot be estimated by the ideas of integration by parts. We are not presenting such a construction, as the formula would be unnecessarily long and does not give new ideas. Finally, we remark that such an entanglement problem, generated by the time-integral reliance of the $U-V$ space techniques, does not show up in the couplings with only $B^{+}$or only $B^{-}$, and does not have to emerge in the $\mathbb{R}^{3} / \mathbb{R}^{4} / \mathbb{T}^{3}$ cases in which $U$ - $V$ spaces are not necessary.

We now prove how the extended KM board game is compatible with the $U-V$ techniques. Given a reference tree, we will create a Duhamel tree (we write ' $D$-tree' for short) to supplement the reference tree. The $D$-tree supplements the given reference tree in the sense that the $D$-tree completely shows the arrangement of the cubic terms $D^{(j)}$, defined in Section 5.1, and one could also read off the integration limits from it as in the given reference tree. The whole point of the $D$-tree is to get these two pieces of information in the same picture, as the proof of compatibility then follows trivially. Of course, from now on, we assume that equation 3.6) has already been plugged in and we are doing the $d t_{k+1}$ integral, which is from 0 to $t_{1}$, last.

Algorithm 7. In the D-tree, we will write each node prefaced by a D. Each node $D^{(j)}$ will have a left child, middle child and right child:

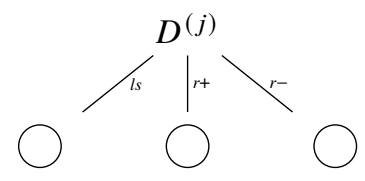

The labelling of $l s, r+$ and $r$ - for the left, middle and right children, respectively, is a shorthand mnemonic for the procedure for determining the children of $D^{(j)}$ by inspecting the reference tree. Apply the following steps for $j=1$ (with no left child), then repeat the steps for all $D^{(j)}$ that appear as children; continue to repeat the steps until all vertices without children are F:

1. To determine the left child of $D^{(j)}$, locate node $j$ in the reference tree and apply the 'left same' rule. If node $j$ in the reference tree is + , and $j+$ has a left child $\ell+($ of the same sign +$)$, then place $D^{(l)}$ as the left child in the D-tree. If the $j$ node in the reference tree is -, and $j-$ has a left child $\ell-($ of the same sign-), then place $D^{(l)}$ as the left child in the D-tree. If node $j$ in the reference tree does not have a left child of the same sign, then place $F$ as the left child of $D^{(j)}$ in the D-tree. 
2. To determine the middle and right children of $D^{(j)}$, locate node $j$ in the reference tree. Examine the right child of $j$ (if it exists), and consider its full left branch

$$
p_{1}+, \ldots, p_{\alpha}+, n_{1}-, \ldots, n_{\beta}-\text {. }
$$

It is possible here that $\alpha=0$ (no + nodes on this left branch), and it is also possible that $\beta=0$ (no - nodes on this left branch). In the D-tree, as the middle child of $D^{(j)}$ place $D^{\left(p_{1}\right)}$, and as the right child of $D^{(j)}$ place $D^{\left(n_{1}\right)}$. If either or both is missing ( $\alpha=0$ or $\beta=0$, respectively), place F instead.

A quick and simple example is the $D$-tree for the integral in Section 5.1:

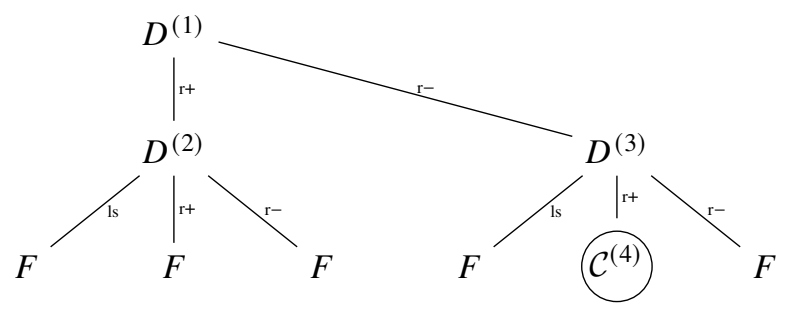

Here is a longer example:

Example 7. Consider the following reference tree:

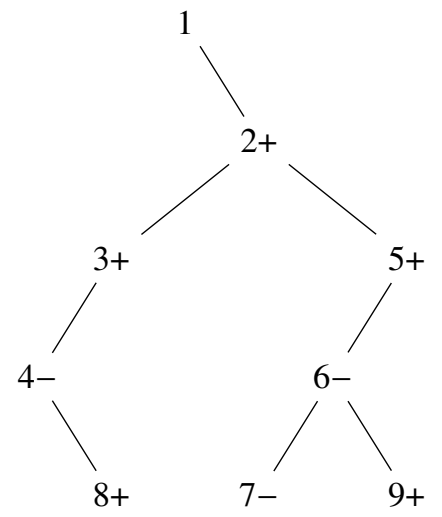

Its supplemental $D$-tree is as follows:

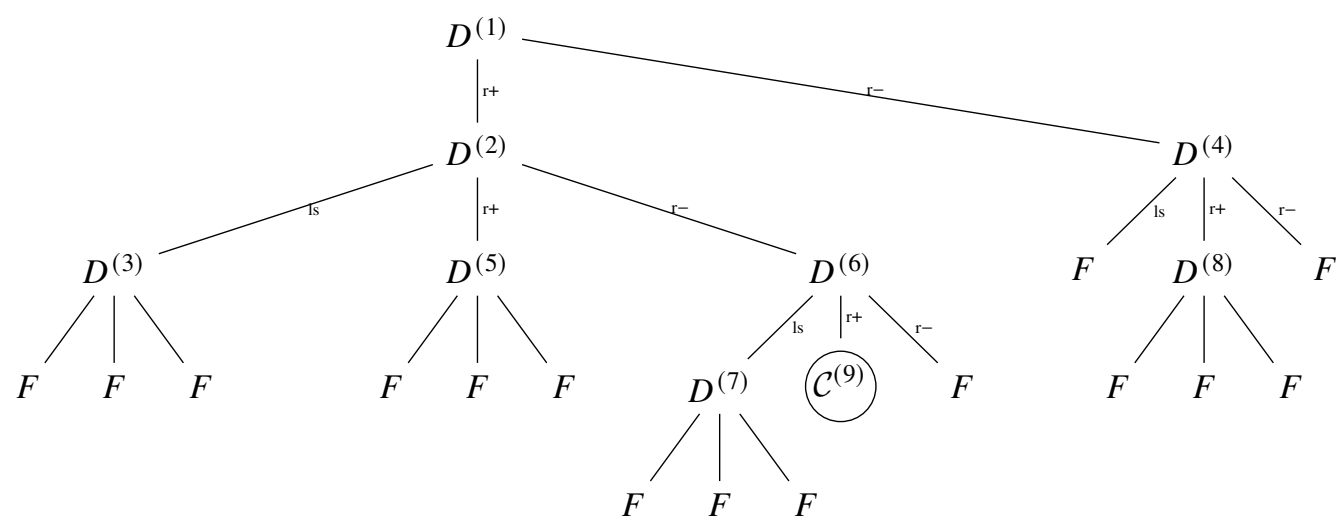

Every bottom node of the form $D^{(j)}$ (as opposed to $F$ ) has implicitly three $F$ children, except for the $D^{(k+1)}$ node, which is special (in our case here, it is $D_{9}$ ). In this case, the $D$-tree was generated as 
follows. Take $D^{(2)}$, for example, in the reference tree.

- To determine the left child of $D^{(2)}$ in the $D$-tree, we look at the reference tree and follow the 'left same' rule. The left child of $2+$ is $3+$, so we place $D^{(3)}$ as the left child of $D^{(2)}$ in the $D$-tree. (If it were 3 - instead, we would place $F$ in the $D$-tree, since the signs are different.)

- To determine the middle child of $D^{(2)}$ in the $D$-tree, we look at the reference tree and follow the 'right +' rule. That is, we take the right child and consider its left branch: 5+, 6-, 7-. We note the first + node, which is $5+$, and assign $D^{(5)}$ as the middle child of $D^{(2)}$. If there were no + node in the left branch, we would have assigned $F$.

- To determine the right child of $D^{(2)}$ in the $D$-tree, we look at the reference tree and follow the 'right -' rule. That is, we take the right child and consider its left branch: 5+, 6-, 7-. We note the first - node, which is 6-, and assign $D^{(6)}$ as the right child of $D_{2}$. If there were no - node in the left branch, we would have assigned $F$.

Proof of compatibility. With the D-tree, we can now read formula (4.14) better. This is because the rule for assigning upper limits of time integration is actually the same rule for constructing children in the $D$-tree. By the construction of the $D$-tree, we can write the form of each $D^{(j)}, j \neq k+1$, and the integration limit for $t_{j}$. If $D^{(j)}$ has children $L, M, R$ (for left, middle and right) and has parent $D^{(l)}$ in the $D$-tree, then (ignoring the role of complex conjugates)

$$
D^{(j)}\left(t_{j}\right)=U\left(-t_{j}\right)\left[\left(U_{j} L\right)\left(U_{j} M\right)\left(U_{j} R\right)\right]
$$

and the integration of $t_{j}$ is exactly from 0 to $t_{l}$. One can directly see from the picture in Example 7 that all Duhamel terms inside a $D^{(j)}$ must have the same integration limit, and they factor. Therefore, there is no entanglement in each stage of the coupling process. An induction then shows that there is no entanglement for any coupling of finite length or stages. Or in other words, the extended KM board game is compatible with the $U-V$ techniques.

For completeness, we finish Example 7 with the integration limits:

Example 8. Continuing Example 7, we have

$$
D^{(2)}=U\left(-t_{2}\right)\left[U_{2} D^{(3)} \cdot U_{2} D^{(5)} \cdot U_{2} D^{(6)}\right] .
$$

The three terms inside this expression are

$$
\begin{aligned}
& D^{(3)}=U\left(-t_{3}\right)\left[U_{3} F\left(t_{9}\right) \cdot U_{3} F\left(t_{9}\right) \cdot U_{3} F\left(t_{9}\right)\right], \\
& D^{(5)}=U\left(-t_{5}\right)\left[U_{5} F\left(t_{9}\right) \cdot U_{5} F\left(t_{9}\right) \cdot U_{5} F\left(t_{9}\right)\right], \\
& D^{(6)}=U\left(-t_{6}\right)\left[U_{6} D^{(7)} \cdot U_{6} D^{(9)} \cdot U_{6} F\left(t_{9}\right)\right],
\end{aligned}
$$

where $F\left(t_{i}\right)=U\left(-t_{i}\right) \phi$. On the other hand, we have

$$
D^{(4)}=U\left(-t_{4}\right)\left[U_{4} F\left(t_{9}\right) \cdot U_{4} D^{(8)} \cdot U_{4} F\left(t_{9}\right)\right] .
$$

Now, read the time integration limits from the reference tree or the $D$-tree; $t_{2}$ and $t_{4}$ have upper limit $t_{1}$, while $t_{3}, t_{5}$ and $t_{6}$ all have upper limit $t_{2}$, and so on. Start by writing $\int_{t_{9}=0}^{t_{1}}$ on the outside. Notice that this makes the inner $t_{6}$ integral start at $t_{9}$ in order to retain the condition $t_{9} \leq t_{6}$ from formula (4.14) and the tree reading. Take all $t_{j}$ integrals for $j=2$ or for which $D^{(j)}$ is a descendant of $D^{(2)}$. This is

$$
\int_{t_{2}=0}^{t_{1}} \int_{t_{3}=0}^{t_{2}} \int_{t_{5}=0}^{t_{2}} \int_{t_{6}=t_{9}}^{t_{2}} \int_{t_{7}=0}^{t_{6}} .
$$


Then collect all $t_{j}$ integrals for $j=4$ or for which $D^{(j)}$ is a descendant of $D^{(4)}$. This is

$$
\int_{t_{4}=0}^{t_{1}} \int_{t_{8}=0}^{t_{4}}
$$

Notice that expressions (5.9) and (5.10) split, by Fubini, since none of the limits of integration in expression (5.9) appear in expression (5.10), and vice versa. So we can write this piece of $\gamma^{(1)}$ as

$$
\gamma^{(1)}\left(t_{1}\right)=\int_{t_{9}=0}^{t_{1}}\left[\int_{t_{2}=0}^{t_{1}} \int_{t_{3}=0}^{t_{2}} \int_{t_{5}=0}^{t_{2}} \int_{t_{6}=t_{9}}^{t_{2}} \int_{t_{7}=0}^{t_{6}} U_{1} D^{(2)}\left(t_{2}, x_{1}\right)\right]\left[\int_{t_{4}=0}^{t_{1}} \int_{t_{8}=0}^{t_{4}} U_{1} D^{(4)}\left(t_{4}, x_{1}^{\prime}\right)\right] .
$$

Write out $D^{(2)}$ as in equation (5.7) and $D^{(4)}$ as in equation (5.8). Notice that we can distribute the integrals $\int_{t_{3}=0}^{t_{2}} \int_{t_{5}=0}^{t_{2}} \int_{t_{6}=0}^{t_{2}}$ onto the $D^{(3)}, D^{(5)}$ and $D^{(6)}$ terms, respectively:

$$
\begin{aligned}
\int_{t_{2}=0}^{t_{1}} \int_{t_{3}=0}^{t_{2}} \int_{t_{5}=0}^{t_{2}} & \int_{t_{6}=t_{9}}^{t_{2}} \int_{t_{7}=0}^{t_{6}} U_{1} D^{(2)}\left(t_{2}, x_{1}\right) \\
& =\int_{t_{2}=0}^{t_{1}} U_{1,2}\left[\left(\int_{t_{3}=0}^{t_{2}} U_{2} D^{(3)}\left(t_{3}\right)\right) \cdot\left(\int_{t_{5}=0}^{t_{2}} U_{2} D^{(5)}\left(t_{5}\right)\right) \cdot\left(\int_{t_{6}=t_{9}}^{t_{2}} \int_{t_{7}=0}^{t_{6}} U_{2} D^{(6)}\left(t_{6}\right)\right)\right] .
\end{aligned}
$$

We have kept the $t_{7}$ integral together with $t_{6}$ because $D^{(7)}$ is a child of $D^{(6)}$ in the $D$-tree. We can see that all the Duhamel structures are fully compatible with the $U-V$ techniques. The rest is similar, and we omit further details.

\subsection{Estimates for general $k$}

As the compatiblity between the extended KM board game and the $U-V$ techniques has been proved in Section 5.2, we can now apply the $U-V$ techniques from Section 5.1 to the general case. We see from Section 5.1 that estimates (5.1) and (5.2) provide gains whenever the $l$ th coupling contracts a $U \phi$. For large $k$, at least $\frac{2}{3} k$ of the couplings carry such a property and thus allow gains.

Definition 5.1. For $l<k$, we say that the $l$ th coupling is an unclogged coupling if the corresponding cubic term $\mathcal{C}^{(l+1)}$ or $D^{(l+1)}$ has contracted at least one $U \phi$ factor. If the $l$ th coupling is not unclogged, we call it a congested coupling.

Lemma 5.2. For large $k$, there are at least $\frac{2}{3} k$ unclogged couplings in $k$ couplings when one plugs equation (3.6) into expression (3.9).

Proof. Assume there are $j$ congested couplings; then there are $(k-1-j)$ unclogged couplings. Before the $(k-1)$ th coupling, there are $2 k-1$ copies of $U \phi$ available. After the first coupling, all of these $2 k-1$ copies of $U \phi$ except one must be inside some Duhamel term. Since the $j$ congested couplings do not consume any $U \phi$, to consume all $2 k-2$ copies of $U \phi$ we have to have

$$
2 k-2 \leq 3(k-1-j),
$$

because a unclogged coupling can consume at most three copies of $U \phi$. This inequality certainly holds only if $j<\frac{k}{3}$. Hence, there are at least $\frac{2 k}{3}$ unclogged couplings.

We can now present the algorithm which proves the general case:

Step 0 Plug equation (3.6) into expression (3.9). $\operatorname{Mark} \mathcal{C}_{R}^{(k+1)}$ and all $D^{(l+1)}$ for $l=1, \ldots, k-1$ per the general rule given in the example and Section 5.1. We obtain 


$$
\begin{gathered}
\left\|\left\langle\nabla_{x_{1}}\right\rangle^{-1}\left\langle\nabla_{x_{1}^{\prime}}\right\rangle^{-1} \int_{I_{2}} \cdots \int_{I_{k}} J_{\mu_{m}, \mathrm{sgn}}^{(k+1)}\left(\gamma^{(k+1)}\right)\left(t_{1}, \underline{t}_{k+1}\right) d \underline{t}_{k+1}\right\|_{L_{t_{1}}^{\infty} L_{x, x^{\prime}}^{2}} \\
\quad \leq \int_{0}^{T} d t_{k+1} \int d\left|\mu_{t_{k+1}}\right|(\phi)\left\|\left(\left\langle\nabla_{x_{1}}\right\rangle^{-1} f^{(1)}\left(t_{1}, x_{1}\right)\right)\left(\left\langle\nabla_{x_{1}^{\prime}}\right\rangle^{-1} g^{(1)}\left(t_{1}, x_{1}^{\prime}\right)\right)\right\|_{L_{t_{1}}^{\infty} L_{x, x^{\prime}}^{2}},
\end{gathered}
$$

which 'factors' into

$$
\begin{aligned}
& \leq \int_{0}^{T} d t_{k+1} \int d\left|\mu_{t_{k+1}}\right|(\phi)\left\|\left\langle\nabla_{x_{1}}\right\rangle^{-1} f^{(1)}\left(t_{1}, x_{1}\right)\right\|_{L_{t_{1}}^{\infty} L_{x}^{2}}\left\|\left\langle\nabla_{x_{1}^{\prime}}\right\rangle^{-1} g^{(1)}\left(t_{1}, x_{1}^{\prime}\right)\right\|_{L_{t_{1}}^{\infty} L_{x^{\prime}}^{2}} \\
& \leq \int_{0}^{T} d t_{k+1} \int d\left|\mu_{t_{k+1}}\right|(\phi)\left\|f^{(1)}\right\|_{X^{-1}}\left\|g^{(1)}\right\|_{X^{-1}}
\end{aligned}
$$

for some $f^{(1)}$ and $g^{(1)}$. Of course, only one of $f^{(1)}$ and $g^{(1)}$ can carry the cubic rough term $\mathcal{C}_{R}^{(k+1)}$, as there is only one, so bump the other one into $X^{1}$. Go to step 1 .

Step 1 Set a counter $l=1$ and go to step 2 .

Step 2 If $D^{(l+1)}$ is a $D_{\phi, R}^{(l+1)}$, apply estimate (5.1), put the factor carrying $\mathcal{C}_{R}^{(k+1)}-$ which will be a $U \mathcal{C}_{R}^{(k+1)}$ or a $D_{R}^{(j+1)}$ for some $j-$ in $X^{-1}$ and replace the $X^{1}$ norm of $U \phi$ by the $H^{1}$ norm of $\phi$; if the ending estimate includes $\left\|U \mathcal{C}_{R}^{(k+1)}\right\|_{X^{-1}}$, replace it by $\left\|\mathcal{C}_{R}^{(k+1)}\right\|_{H^{-1}}$. Then go to step 6. If $D^{(l+1)}$ is not a $D_{\phi, R}^{(l+1)}$, go to step 3 .

Step 3 If $D^{(l+1)}$ is a $D_{\phi}^{(l+1)}$, apply estimate (5.2) and replace the $X^{1}$ norm of $U \phi$ by the $H^{1}$ norm of $\phi$. Then go to step 6. If $D^{(l+1)}$ is not a $D_{\phi}^{(l+1)}$, go to step 4 .

Step 4 If $D^{(l+1)}$ is a $D_{R}^{(l+1)}$, apply estimate (5.3), put the factor carrying $\mathcal{C}_{R}^{(k+1)}$ - which will be a $U \mathcal{C}_{R}^{(k+1)}$ or a $D_{R}^{(j+1)}$ for some $j-$ in $X^{-1}$ and replace the $X^{1}$ norm of $U \phi$ by the $H^{1}$ norm of $\phi$; if the ending estimate includes $\left\|U \mathcal{C}_{R}^{(k+1)}\right\|_{X^{-1}}$, replace it by $\left\|\mathcal{C}_{R}^{(k+1)}\right\|_{H^{-1}}$. Then go to step 6. If $D^{(l+1)}$ is not a $D_{R}^{(l+1)}$, go to step 5 .

Step 5 If $D^{(l+1)}$ is a $D^{(l+1)}$, apply estimate (5.4) and replace the $X^{1}$ norm of $U \phi$ by the $H^{1}$ norm of $\phi$. Then go to step 6.

Step 6 Set the counter $l=l+1$. If $l<k$, go to step 2; otherwise go to step 7 .

Step 7 Replace all the leftover $\|U \phi\|_{X^{1}}$ by $\|\phi\|_{H^{1}}$. There is actually at most one leftover $\|U \phi\|_{X^{1}}$, which is exactly $f^{(1)}$ or $g^{(1)}$ from the beginning and only happens when the sign $J_{\mu_{m} \text {,sgn }}^{(k+1)}$ under consideration is all + or all - . As it is not inside any Duhamel, it is not taken care of by steps $1-6$. Go to step 8.

Step 8 We are now at the $k$ th coupling, and have applied formulas (5.1) and (5.2) at least $\frac{2}{3} k$ times; thus we are looking at

$$
\begin{aligned}
& \left\|\left\langle\nabla_{x_{1}}\right\rangle^{-1}\left\langle\nabla_{x_{1}^{\prime}}\right\rangle^{-1} \int_{I_{2}} \cdots \int_{I_{k}} J_{\mu_{m}, \mathrm{sgn}}^{(k+1)}\left(\gamma^{(k+1)}\right)\left(t_{1}, \underline{t}_{k+1}\right) d \underline{t}_{k+1}\right\|_{L_{t_{1}}^{\infty} L_{x, x^{\prime}}^{2}} \\
\leq & C^{k-1} \int_{0}^{T} d t_{k+1} \int d\left|\mu_{t_{k+1}}\right|(\phi)\|\phi\|_{H^{1}}^{\frac{4}{3} k-1}\left(T^{\frac{1}{7}} M_{0}^{\frac{3}{5}}\left\|P_{\leq M_{0}} \phi\right\|_{H^{1}}+\left\|P_{>M_{0}} \phi\right\|_{H^{1}}\right)^{\frac{2}{3} k}\left\||\phi|^{2} \phi\right\|_{H^{-1}} .
\end{aligned}
$$

Applying the 4D Sobolev (5.5) to the rough factor yields

$$
\leq C^{k} \int_{0}^{T} d t_{k+1} \int d\left|\mu_{t_{k+1}}\right|(\phi)\|\phi\|_{H^{1}}^{\frac{4}{3} k+2}\left(T^{\frac{1}{7}} M_{0}^{\frac{3}{5}}\left\|P_{\leq M_{0}} \phi\right\|_{H^{1}}+\left\|P_{>M_{0}} \phi\right\|_{H^{1}}\right)^{\frac{2}{3} k} .
$$


Putting in the support property (3.5) gives

$$
\begin{aligned}
& \leq \int_{0}^{T} d t_{k+1} \int d\left|\mu_{t_{k+1}}\right|(\phi) C^{k} C_{0}^{\frac{4}{3} k+2}\left(T^{\frac{1}{7}} M_{0}^{\frac{3}{5}} C_{0}+\varepsilon\right)^{\frac{2}{3} k} \\
& \leq 2 T C^{k} C_{0}^{\frac{4}{3} k+2}\left(T^{\frac{1}{7}} M_{0}^{\frac{3}{5}} C_{0}+\varepsilon\right)^{\frac{2}{3} k} \\
& \leq 2 T C_{0}^{2}\left(C C_{0}^{3} T^{\frac{1}{7}} M_{0}^{\frac{3}{5}}+C C_{0}^{2} \varepsilon\right)^{\frac{2}{3} k},
\end{aligned}
$$

as claimed.

Thus, we have proved Proposition 3.7 and hence Theorem 3.1. As mentioned before, the main theorem (Theorem 1.1) then follows from Theorem 3.1 and Lemma 3.3, which checks Theorem 3.1(c) for solutions of equation (1.2) generated by equation (1.1) via equation (1.3).

\section{Appendix A. Some further remarks}

After reading the main part of the paper, it should now be clear that the proof of Theorem 1.1 goes through if the $\mathbb{T}^{4}$ energy-critical problem is replaced by the corresponding problems on $\mathbb{R}^{3}, \mathbb{R}^{4}$ or $\mathbb{T}^{3}$. (The method in this paper also provides a unified proof for the unconditional uniquness of the $H^{1}$-supercritical NLS [26].) One could use the analysis in Theorem 3.1 but slightly different logic to conclude another form of Theorem 1.1, stated as Theorem A.1. It is certainly equivalent to Theorem 1.1 , but its format is more closely related to well-posedness results.

Theorem A.1. Every $C_{[0, T]}^{0} H_{x}^{1}$ solution to equation (1.1) is also in $X^{1}$.

Proof. We give only a sketch, as this route is only slightly different in the logic and is in fact messy in estimates, because it needs to run the main argument twice, one part of which is in a weaker space.

1. Plug the chosen $C_{[0, T]}^{0} H_{x}^{1}$ solution $u$ directly (not taking a difference) into equation (3.4) and iterate.

2. Group the terms in the Duhamel-Born expansion into free and interaction parts like in [17, 19, 21, 25].

3. Apply the analysis of Theorem 3.1 to deduce that the interaction part is zero in $L_{t}^{\infty} H_{x, x^{\prime}}^{-1}$. That is, $|u\rangle\langle u|$ equals the free part.

4. Apply the analysis of Theorem 3.1 again, but in $X^{1}$, to conclude that the free part (and hence $u$ ) is in $X^{1}$.

On the other hand, we remark that the $\mathbb{T}^{4}$ case is a bit 'special' in the aspect of multilinear estimates. The stronger $L_{t}^{1} H_{x}^{s}$ versions of formulas (2.7) and (2.11) can be proved easily on $\mathbb{R}^{3}$ [39] and $\mathbb{R}^{4}$, and with a highly technical and careful argument on $\mathbb{T}^{3}$ [24]. In fact, here is a short proof for the $\mathbb{R}^{4}$ case.

Lemma A.2. On $\mathbb{R}^{4}$,

$$
\begin{gathered}
\left\|e^{i t \Delta} f_{1} e^{i t \Delta} f_{2} e^{i t \Delta} f_{3}\right\|_{L_{T}^{1} H^{-1}} \\
\|\| f_{1}\left\|_{H^{-1}}\right\| f_{2}\left\|_{H^{1}}\right\| f_{3} \|_{H^{1}} \\
\left\|e^{i t \Delta} f_{1} e^{i t \Delta} f_{2} e^{i t \Delta} f_{3}\right\|_{L_{T}^{1} H^{1}} \lesssim\left\|f_{1}\right\|_{H^{1}}\left\|f_{2}\right\|_{H^{1}}\left\|f_{3}\right\|_{H^{1}} .
\end{gathered}
$$

In particular, these formulas imply formulas (2.7) and (2.11). That is, formulas (1) and (2) are indeed stronger than formulas (2.7) and (2.11). 
Proof. We prove only formula (1), as formula (2) follows similarly. Given $g \in L_{t}^{\infty} H_{x}^{-1}$, we have

$$
\left|\int e^{i t \Delta} f_{1} e^{i t \Delta} f_{2} e^{i t \Delta} f_{3} g d t d x\right| \leq\left\||\nabla|^{-1} e^{i t \Delta} f_{1}\right\|_{L_{t, x}^{3}}\left\||\nabla|\left(e^{i t \Delta} f_{2} e^{i t \Delta} f_{3} g\right)\right\|_{L_{t, x}^{\frac{3}{2}}},
$$

where

$$
\begin{aligned}
\left\||\nabla|\left(e^{i t \Delta} f_{2} e^{i t \Delta} f_{3} g\right)\right\|_{L_{t, x}^{\frac{3}{2}}} \lesssim & \left\|\left(|\nabla| e^{i t \Delta} f_{2}\right) e^{i t \Delta} f_{3} g\right\|_{L_{t, x}^{\frac{3}{2}}}+\left\|(|\nabla| g) e^{i t \Delta} f_{2} e^{i t \Delta} f_{3}\right\|_{L_{t, x}^{\frac{3}{2}}} \\
\lesssim & \left\||\nabla| e^{i t \Delta} f_{2}\right\|_{L_{t, x}^{3}}\left\|e^{i t \Delta} f_{3}\right\|_{L_{t}^{3} L_{x}^{12}}\|g\|_{L_{t}^{\infty} L_{x}^{4}} \\
& +\left\|e^{i t \Delta} f_{2}\right\|_{L_{t}^{3} L_{x}^{12}}\left\|e^{i t \Delta} f_{3}\right\|_{L_{t}^{3} L_{x}^{12}}\|\nabla g\|_{L_{t}^{\infty} L_{x}^{2}} \\
\lesssim & \left\|f_{2}\right\|_{H^{1}}\left\|f_{3}\right\|_{H^{1}}\|g\|_{L_{t}^{\infty} H_{x}^{1}}
\end{aligned}
$$

We see that formulas (1) and (2) are indeed elementary to prove if one has the $L_{t, x}^{3}$ estimate, which is known to fail on $\mathbb{T}^{4}$ [2]. Scale-invariant $\mathbb{T}^{4} L_{t}^{3}$-Strichartz estimates with derivatives are also absent in the literature. That is, proving formulas (1) and (2) on $\mathbb{T}^{4}$ would be very difficult, and they may not even be true. In fact, if both formulas (1) and (2) hold on $\mathbb{T}^{4}$, we can deduce that $\left\|e^{i t \Delta} P_{\leq M} f\right\|_{L_{t}^{3} L_{x}^{6}} \lesssim M^{\frac{2}{3}}\left\|P_{\leq M} f\right\|_{L^{2}}$, which is a $\mathbb{T}^{4}$ scale-invariant estimate carrying the $L_{t}^{3}$ exponent and may not be true. Hence, we see that the $\mathbb{T}^{4}$ case is indeed 'special' in the aspect of multilinear estimates, and we are forced to use the weaker $U-V$ space estimates to be on the safe side.

On the other hand, we remark that as estimates (1) and (2) were proved using Hölder, Strichartz and so on with $\geq 2$ time exponents, using $\left\|\int_{0}^{t} e^{i(t-s) \Delta} f(s) d s\right\|_{X^{1}} \leq\|f\|_{L_{t}^{1} H_{x}^{1}}$ and the inclusion $\|f\|_{U^{p}} \lesssim\|f\|_{U^{2}}$ for $p \geq 2$, formulas (2.7) and (2.11) are reduced on $\mathbb{R}^{4}$ from formulas (1) and (2) by applying the atomic structure of $U^{p}$ on the nuts and bolts. We omit the details but remark that one would get a $U^{1}$ estimate instead if one applied the atomic structure directly on the $L_{t}^{1}$ estimate. That is, one could have multiple versions of multilinear estimates yielding existence. For the moment, let us consider the $\mathbb{T}^{3}$ quintic problem as an example, since $\mathbb{R}^{3} / \mathbb{R}^{4}$ are simpler and $\mathbb{T}^{4}$ may not allow the ambiguity to be mentioned. Instead of using the $\mathbb{T}^{3}$ versions of formulas (2.7) and (2.11), one could use the $\mathbb{T}^{3}$ versions of formulas (1) and (2), which do not need $U-V$ techniques, or the $U^{1}$ versions of their implications to show local existence for the $\mathbb{T}^{3}$ quintic problem in three similar but different subspaces of $H^{1}$. The only way to know if these three versions yield the same solution is an unconditional-uniqueness theorem.

Finally, separate from answering the original mathematical problem that there could be multiple solutions coming from different spaces in which equation (1.1) is well posed, the unconditional-uniqueness problems on $\mathbb{T}^{n}$ have practical applications. An example is the control problem for the Lugiato-Lefever system, first formulated in [52], which could be considered as an NLS with forcing:

$$
\begin{aligned}
i \partial_{t} u_{f} & =-\Delta u_{f} \pm\left|u_{f}\right|^{p-1} u_{f}+f \text { in } \mathbb{R} \times \mathbb{T}^{n}, \\
u_{f}(0, x) & =u_{0} .
\end{aligned}
$$

The problem is to find $f$ and $u_{0}$ such that $u_{f} \in X$, for some space $X$ in which equation (3) is well posed, minimises some given functional $Z(u)$. For some experimental and engineering purposes, the spatial domain has to be $\mathbb{T}^{n}$. The space $X$ in which one looks for the minimiser largely determines the difficulty. If $X=L_{x}^{2}$ or $H_{x}^{1}$, there are techniques readily available to hunt for minimisers. However, how to search for minimisers when $X$ is a proper subspace of $H_{x}^{1}$, like $H_{x}^{2}$ or $H_{x}^{1} \cap L_{t}^{p} L_{x}^{q}$ - a common space for wellposedness - remains open. Such a dilemma can be resolved if one has unconditional-uniqueness results like Theorem 1.1. 
Acknowledgments. The first author would like to thank Manoussos Grillakis, Matei Machedon and Changxing Miao for enthusiastic discussions related to this work. Moreover, the authors would like to thank Shunlin Shen and the referees for their careful reading and checking of the paper and their insightful comments and helpful suggestions, which have made the paper better.

Funding statement. The first author was supported in part by NSF grant DMS-2005469. The second author was partially supported by NSF grant DMS-2055072.

Competing Interests. None.

\section{References}

[1] R. Adami, F. Golse and A. Teta, 'Rigorous derivation of the cubic NLS in dimension one', J. Stat. Phys. 127 (2007), 1194-1220.

[2] J. Bourgain, 'Fourier transform restriction phenomena for certain lattice subsets and applications to nonlinear evolution equations, part I: Schrödinger dquations', Geom. Funct. Anal. 3 (1993), 107-156.

[3] J. Bourgain, 'Global well-posedness of defocusing 3D critical NLS in the radial case', J. Amer. Math. Soc. 12 (1999), $145-171$.

[4] J. Bourgain and C. Demeter, 'The proof of the $l^{2}$ decoupling conjecture', Ann. of Math. (2) 182 (2015), 351-389.

[5] T. Chen, R. Denlinger and N. Pavlović, 'Local well-posedness for Boltzmann's equation and the Boltzmann hierarchy via Wigner transform', Comm. Math. Phys. 368 (2019), 427-465.

[6] T. Chen, C. Hainzl, N. Pavlović and R. Seiringer, 'Unconditional uniqueness for the cubic Gross-Pitaevskii hierarchy via quantum de Finetti', Comm. Pure Appl. Math. 68 (2015), 1845-1884.

[7] T. Chen and N. Pavlović, 'On the Cauchy problem for focusing and defocusing Gross-Pitaevskii hierarchies', Discrete Contin. Dyn. Syst. 27 (2010), 715-739.

[8] T. Chen and N. Pavlović, 'The quintic NLS as the mean field limit of a boson gas with three-body interactions', J. Funct. Anal. 260 (2011), 959-997.

[9] T. Chen and N. Pavlović, 'A new proof of existence of solutions for focusing and defocusing Gross-Pitaevskii hierarchies', Proc. Amer. Math. Soc. 141 (2013), 279-293.

[10] T. Chen and N. Pavlović, 'Higher order energy conservation and global wellposedness of solutions for Gross-Pitaevskii hierarchies', Commun. PDE, 39 (2014), 1597-1634.

[11] T. Chen and N. Pavlović, Derivation of the cubic NLS and Gross-Pitaevskii hierarchy from manybody dynamics in $d=3$ based on spacetime norms, Ann. H. Poincare, 15 (2014), 543-588.

[12] T. Chen, N. Pavlović and N. Tzirakis, 'Energy conservation and blowup of solutions for focusing Gross-Pitaevskii hierarchies', Ann. Inst. H. Poincaré Anal. Non Linéaire 27 (2010), 1271-1290.

[13] T. Chen and K. Taliaferro, Derivation in Strong Topology and Global Well-posedness of Solutions to the Gross-Pitaevskii Hierarchy, Comm. Partial Differential Equations 39 (2014), 1658-1693.

[14] X. Chen, 'Classical proofs of Kato type smoothing estimates for the Schrödinger equation with quadratic potential in $R^{n+1}$ with application', Differential Integral Equations 24 (2011), 209-230.

[15] X. Chen, 'Second order corrections to mean field evolution for weakly interacting bosons in the case of three-body interactions', Arch. Ration. Mech. Anal. 203 (2012), 455-497.

[16] X. Chen, 'Collapsing estimates and the rigorous derivation of the $2 \mathrm{~d}$ cubic nonlinear Schrödinger equation with anisotropic switchable quadratic traps', J. Math. Pures Appl. (9) 98 (2012), 450-478.

[17] X. Chen, 'On the rigorous derivation of the 3D cubic nonlinear Schrödinger equation with a quadratic trap', Arch. Ration. Mech. Anal. 210 (2013), 365-408.

[18] X. Chen and J. Holmer, 'On the rigorous derivation of the 2D cubic nonlinear Schrödinger equation from 3D quantum many-body dynamics', Arch. Ration. Mech. Anal. 210 (2013), 909-954.

[19] X. Chen and J. Holmer, 'On the Klainerman-Machedon conjecture of the quantum BBGKY hierarchy with self-interaction', J. Eur. Math. Soc. (JEMS) 18 (2016), 1161-1200.

[20] X. Chen and J. Holmer, 'Focusing quantum many-body dynamics: The rigorous derivation of the 1D focusing cubic nonlinear Schrödinger equation, Arch. Ration. Mech. Anal. 221 (2016), 631-676.

[21] X. Chen and J. Holmer, 'Correlation structures, many-body scattering processes and the derivation of the Gross-Pitaevskii hierarchy, Int. Math. Res. Not. IMRN 2016 (2016), 3051-3110.

[22] X. Chen and J. Holmer, 'Focusing quantum many-body dynamics II: The rigorous derivation of the 1D focusing cubic nonlinear Schrödinger equation from 3D', Anal. PDE 10 (2017), 589-633.

[23] X. Chen and J. Holmer, 'The rigorous derivation of the 2D cubic focusing NLS from quantum many-body evolution', Int. Math. Res. Not. IMRN 2017 (2017), 4173-4216.

[24] X. Chen and J. Holmer, 'The derivation of the energy-critical NLS from quantum many-body dynamics', Invent. Math. 217 (2019), 433-547.

[25] X. Chen and J. Holmer, 'Quantitative derivation and scattering of the 3D cubic NLS in the energy space', Preprint, 2021, arXiv:2104.06086. 
[26] X. Chen, S. Shen and Z. Zhang, 'The unconditional uniqueness for the energy-supercritical NLS', Preprint, 2021, arXiv:2104.06592.

[27] X. Chen and P. Smith, 'On the unconditional uniqueness of solutions to the infinite radial Chern-Simons-Schrödinger hierarchy', Anal. PDE 7 (2014), 1683-1712.

[28] J. Colliander, M. Keel, G. Staffilani, H. Takaoka and T. Tao, 'Global well-posedness and scattering for the energy-critical nonlinear Schrödinger equation in $\mathbb{R}^{3}$, Ann. of Math. (2) 167 (2008), 767-865.

[29] L. Erdôs, B. Schlein and H. T. Yau, 'Derivation of the cubic non-linear Schrödinger equation from quantum dynamics of many-body systems', Invent. Math. 167 (2007), 515-614.

[30] L. Erdôs, B. Schlein and H. T. Yau, 'Rigorous derivation of the Gross-Pitaevskii equation with a large interaction potential', J. Amer. Math. Soc. 22 (2009), 1099-1156.

[31] L. Erdős, B. Schlein and H. T. Yau, 'Derivation of the Gross-Pitaevskii equation for the dynamics of Bose-Einstein condensate', Ann. of Math. (2) 172 (2010), 291-370.

[32] P. Gressman, V. Sohinger and G. Staffilani, 'On the uniqueness of solutions to the periodic 3D Gross-Pitaevskii hierarchy', J. Funct. Anal. 266 (2014), 4705-4764.

[33] M. Grillakis, 'On nonlinear Schrödinger equations', Comm. Partial Differential Equations 25 (2000), 1827-1844.

[34] S. Herr and V. Sohinger, 'The Gross-Pitaevskii hierarchy on general rectangular tori', Arch. Ration. Mech. Anal., 220 (2016), 1119-1158.

[35] S. Herr and V. Sohinger, 'Unconditional uniqueness results for the nonlinear Schrödinger equation', Commun. Contemp. Math. 21 (2019), 1850058.

[36] S. Herr, D. Tataru and N. Tzvetkov, 'Global well-posedness of the energy critical Nonlinear Schrödinger equation with small initial data in $H^{1}\left(T^{3}\right)$ ', Duke Math. J. 159 (2011), 329-349.

[37] S. Herr, D. Tataru and N. Tzvetkov, 'Strichartz estimates for partially periodic solutions to Schrödinger equations in 4d and applications', J. Reine Angew. Math. 690 (2014) 65-78.

[38] Y. Hong, K. Taliaferro and Z. Xie, 'Unconditional uniqueness of the cubic Gross-Pitaevskii Hierarchy with low regularity', SIAM J. Math. Anal., 47 (2015), 3314-3341.

[39] Y. Hong, K. Taliaferro and Z. Xie, 'Uniqueness of solutions to the 3D quintic Gross-Pitaevskii hierarchy', J. Funct. Anal. 270(1) (2016), 34-67.

[40] A. D. Ionescu and B. Pausader, 'The energy-critical defocusing NLS on $T^{3}$, Duke Math. J. 161 (2012), 1581-1612.

[41] T. Kato, 'On nonlinear Schrödinger equations, II. $H^{S}$-solutions and unconditional well-posedness', J. Anal. Math. 67 (1995), 281-306.

[42] C. Kenig and F. Merle, 'Global well-posedness, scattering and blow-up for the energy-critical, focusing, non-linear Schrödinger equation in the radial case', Invent. Math. 166 (2006), 645-675.

[43] R. Killip and M. Vişan, 'Scale invariant Strichartz estimates on tori and applications', Math. Res. Lett. 23 (2016), 445-472.

[44] K. Kirkpatrick, B. Schlein and G. Staffilani, 'Derivation of the two dimensional nonlinear Schrödinger equation from many body quantum dynamics', Amer. J. Math. 133 (2011), 91-130.

[45] N. Kishimoto, 'Unconditional local well-posedness for periodic NLS', Preprint, 2019, arXiv:1912.12704.

[46] S. Klainerman and M. Machedon, 'Space-time estimates for null forms and the local existence theorem', Comm. Pure Appl. Math. 46 (1993), 1221-1268.

[47] S. Klainerman and M. Machedon, 'On the uniqueness of solutions to the Gross-Pitaevskii hierarchy', Comm. Math. Phys. 279 (2008), 169-185.

[48] H. Koch and D. Tataru, 'Dispersive estimates for principally normal pseudodifferential operators', Comm. Pure Appl. Math. 58(2) (2005), 217-284.

[49] H. Koch and D. Tataru, 'A priori bounds for the 1D cubic NLS in negative Sobolev spaces', Int. Math. Res. Not. IMRN 2007 (2007), rnm053.

[50] H. Koch, D. Tataru and M. Vişan, Dispersive Equations and Nonlinear Waves, Oberwolfach Seminars vol. 45 (Birkhäuser, Basel, 2014).

[51] M. Lewin, P. T. Nam and N. Rougerie, 'Derivation of Hartree's theory for generic mean-field Bose systems', Adv. Math. 254 (2014), 570-621.

[52] L. A. Lugiato and R. Lefever, 'Spatial dissipative structures in passive optical systems', Phys. Rev. Lett. 58 (1987), 2209-2211.

[53] D. Mendelson, A. Nahmod, N. Pavlović, M. Rosenzweig and G. Staffilani, 'A rigorous derivation of the Hamiltonian structure for the nonlinear Schrödinger equation', Adv. Math. 365 (2020), 107054.

[54] D. Mendelson, A. Nahmod, N. Pavlović, M. Rosenzweig and G. Staffilani, 'Poisson commuting energies for a system of infinitely many bosons', Preprint, 2019, arXiv:1910.06959.

[55] D. Mendelson, A. Nahmod, N. Pavlović and G. Staffilani, 'An infinite sequence of conserved quantities for the cubic GrossPitaevskii hierarchy on R', Trans. Amer. Math. Soc. 371 (2019), 5179-5202.

[56] E. Ryckman and M. Vişan, 'Global well-posedness and scattering for the defocusing energy-critical nonlinear Schrödinger equation in $\mathbb{R}^{1+4}$, Amer. J. Math. 129 (2007), 1-60. 
[57] V. Sohinger, 'A rigorous derivation of the defocusing cubic nonlinear Schrödinger equation on $T^{3}$ from the dynamics of many-body quantum systems', Ann. Inst. H. Poincaré Anal. Non Linéaire 32 (2015), 1337-1365.

[58] V. Sohinger, 'Local existence of solutions to randomized Gross-Pitaevskii hierarchies', Trans. Amer. Math. Soc. 368 (2016), 1759-1835.

[59] V. Sohinger and G. Staffilani, 'Randomization and the Gross-Pitaevskii hierarchy', Arch. Ration. Mech. Anal. 218 (2015), $417-485$.

[60] Z. Xie, 'Derivation of a nonlinear Schrödinger equation with a general power-type nonlinearity in $d=1,2$ ', Differential Integral Equations 28 (2015), 455-504.

[61] H. Yue, 'Global well-posedness of the energy-critical nonlinear Schrödinger equation on $T^{4}$, Preprint, 2018, arXiv: 1805.09816. 